TRANSACTIONS OF THE

AMERICAN MATHEMATICAL SOCIETY

Volume 364, Number 8, August 2012, Pages 4369-4424

S 0002-9947(2012)05606-6

Article electronically published on March 29, 2012

\title{
TRANSMISSION BOUNDARY PROBLEMS FOR DIRAC OPERATORS ON LIPSCHITZ DOMAINS AND APPLICATIONS TO MAXWELL'S AND HELMHOLTZ'S EQUATIONS
}

\author{
EMILIO MARMOLEJO-OLEA, IRINA MITREA, MARIUS MITREA, AND QIANG SHI
}

\begin{abstract}
The transmission boundary value problem for a perturbed Dirac operator on arbitrary bounded Lipschitz domains in $\mathbb{R}^{3}$ is formulated and solved in terms of layer potentials of Clifford-Cauchy type. As a byproduct of this analysis, an elliptization procedure for the Maxwell system is devised which allows us to show that the Maxwell and Helmholtz transmission boundary value problems are well-posed as a corollary of the unique solvability of this more general Dirac transmission problem.
\end{abstract}

\section{INTRODUCTION}

The treatment of the fundamental boundary value problems arising in mathematical physics via integral equation methods has a rich history and has been a driving force behind the development of much of the modern theory of singular integral operators, as well as certain basic aspects of functional analysis. A case in point is the very genesis of Fredholm theory, which is rooted in attempts to use the compactness of various double layer potential operators on smooth domains in order to solve such boundary value problems. Indeed, as early as 1900, in a paper based on his 1898 doctoral dissertation, E.I. Fredholm initiated the use of such an approach and proved his celebrated Fredholm alternative theorems for the integral equations associated with the Dirichlet problem for the Laplace operator. Moreover, in 1906, Fredholm extended the use of such potential theoretic methods to the case of systems of PDE's by solving the first basic problem of elasticity theory.

In the intervening years, thanks to the successful efforts of many prominent mathematicians, including S. Mikhlin, A.P. Calderón, A. Zygmund and many others, the method of integral operators has become a basic tool in the modern arsenal of techniques used to treat elliptic boundary value problems. Such developments have received further impetus through the program outlined by A.P. Calderón in his 1978 ICM plenary address in which he advocates the use of layer potentials "for much more general elliptic systems [than the Laplacian]"; see [Ca80, p. 90]. An excellent exposition of the state of the art in the area of harmonic analysis techniques for elliptic boundary value problems, up to the early 1990s, can be found in the C. Kenig monograph Ke94.

Received by the editors March 13, 2010 and, in revised form, April 15, 2011.

2010 Mathematics Subject Classification. Primary: 30G35, 35C15, 35F15, 35J56, 42B20, 42B30, 42B37; Secondary 30E20, 31B10, 35F45, 35J25, 45B05, 65N80.

Key words and phrases. Transmission boundary value problems, Lipschitz domains, Dirac operator, Maxwell system, Helmholtz operator, Hardy spaces, Cauchy operator, boundary layer potentials, Clifford algebras, Clifford analysis. 
Layer potential techniques in electromagnetism were pioneered by C. Müller, H. Weyl, and A.P. Calderón in the early 1950s (see [Mu51, We52a, We52b], and Ca54], as well as the subsequent exposition in the monograph [Mu69]). In the incipient stages of this theory, the smoothness assumptions were rather strong (e.g., domains with real analytical boundaries in [Mu51-Mu69, domains of class $C^{8}$ in We52a, and of class $C^{3}$ in Ca54], and one of the ensuing issues was that of clarifying the minimal amount of smoothness required of the boundary of the scatterer ensuring the successful applicability of this approach. In Ca77, Calderón initiated a breakthrough, proving $L^{p}$-boundedness of the Cauchy integral operators on Lipschitz curves with small Lipschitz constant. This was used in [FJR78 by E. Fabes, M. Jodeit, and N. Rivière to solve the classical BVP's for the Laplacian in $C^{1}$ domains. Calderón's result was subsequently sharpened by $\mathrm{R}$. Coifman, A. McIntosh and Y. Meyer in CMM82, who extended Calderón's estimate for the Cauchy integral to general Lipschitz surfaces.

This opened the door for extending the applicability of the method of boundary layer potentials for solving PDE's in domains whose boundaries are merely Lipschitz surfaces. Subsequent, ground-breaking work by B. Dahlberg, E. Fabes, C. Kenig, and G. Verchota in the late 1970s and 1980s in connection with other basic problems arising in mathematical physics (cf. DK87, DK90, DKV88, [FKV88], Ve84]) has shown that large classes of elliptic PDE's, including systems, can be successfully treated via boundary layer methods in arbitrary Lipschitz domains. The methods and techniques pioneered in the aforementioned papers have then been extended to the case of parabolic PDE's in Lipschitz cylinders by R. Brown and Z. Shen in Br89, Sh91.

However, the problem of dealing with the Maxwell system in Lipschitz domains remained open in the early 1990s as D. Colton and R. Kress wrote in their monograph [CK92]: "A major drawback of the integral equation approach to constructively proving the existence of solutions for scattering problems is the relatively strong regularity assumption on the boundary to be of class $C^{2}[\cdots]$. The situation changes considerably if the boundary is allowed to have edges and corners [...]." This issue was addressed in Mi94, where one-sided (i.e., interior and exterior) boundary value problems for the Maxwell system formulated in arbitrary Lipschitz domains in the three-dimensional setting were solved when the boundary data belong to appropriate $L^{p}$-based, Sobolev-like, boundary smoothness spaces, with $p \in(2-\varepsilon, 2+\varepsilon)$. Here, $\varepsilon>0$ is a (typically small) parameter which depends only on the Lipschitz character of the underlying domain. The method employed in Mi94 is that of boundary layer potential operators, and one of the key steps is proving the invertibility of a boundary principal-value singular integral operator of the form $\frac{1}{2} I+M_{k}$ on the aforementioned boundary smoothness spaces when $2-\varepsilon<p<2+\varepsilon$, where $M_{k}$ is the so-called magnetic dipole operator associated with the wave number $k$.

Shortly thereafter, in JM95, this approach was extended to treat scattering phenomena in the higher-dimensional setting in Lipschitz domains for the range $2-\varepsilon<p<2+\varepsilon$. Here it was also shown that if $\partial \Omega \in C^{1}$, then any $p \in$ $(1, \infty)$ will suffice. The issue of identifying the optimal range of $p$ 's for which one-sided boundary value problems for the Maxwell system are well-posed in the setting described in the previous paragraph was taken up in [MMP97, where the authors have shown that, for Lipschitz domains in $\mathbb{R}^{3}$, the interior/exterior Maxwell 
problems are always well-posed as long as $1<p<2+\varepsilon$. This remains an active area of research, and the question of identifying the optimal range of $p$ 's ensuring the unique solvability of the Maxwell boundary value problem formulated in the class of Lipschitz domains in $\mathbb{R}^{m}$, with $m \geq 4$, remains open at the moment.

The goal of the present paper is to continue this line of work and derive estimates in the nature of best possible for the transmission boundary value problem for the three-dimensional Maxwell system. The underlying domain $\Omega$ is assumed to be Lipschitz, and the boundary data are selected from appropriate $L^{p}$-based, Sobolev-like, boundary smoothness spaces. In the case of the Laplace operator, such problems have been solved in [EM04 by relying on the Serrin-Weinberger asymptotic theory (i.e., De Giorgi-Nash-Moser theory at infinity). Subsequently, a new approach for the main results in EM04 has been developed in MMS06 based on the Hölder regularity of the Neumann function associated with the transmission problem for variable coefficient, second order, elliptic (scalar) equations. Here we further extend the scope of the work in [EM04, MMS06, by considering systems of equations. The case of the transmission boundary value problems for the Stokes system have recently been dealt with in [MW10] using techniques somewhat akin to those used in EM04. Transmission boundary value problems for the Lamé operator can also be handled along the lines of the theory developed in MW10. By way of contrast, there are several important technical differences between the treatment of the Stokes and Lamé systems, on the one hand, and the treatment we present here for the Maxwell system, on the other hand. We plan to elaborate on this point later. For now, we wish to introduce notation which allows us to state the main results in this paper. Let $\Omega \subset \mathbb{R}^{3}$ be a bounded Lipschitz domain and set

$$
\Omega_{+}:=\Omega \quad \text { and } \quad \Omega_{-}:=\mathbb{R}^{3} \backslash \bar{\Omega} .
$$

Recall that the propagation of an electromagnetic wave $(E, H)$ in the exterior of an obstacle occupying the region of $\mathbb{R}^{3}$ inside $\Omega$ is governed by the three-dimensional (time-harmonic version of the) Maxwell system

$$
\operatorname{curl} E-i k H=0 \text { in } \Omega_{-}, \quad \operatorname{curl} H+i k E=0 \text { in } \Omega_{-},
$$

plus a suitable decay condition at infinity, whose formulation depends on the nature of the wave number $k \in \mathbb{C}$. Specifically, when $\operatorname{Im} k>0$, then $E, H$ decay exponentially at infinity, whereas if $k \in \mathbb{R} \backslash\{0\}$, then $E, H$ are assumed to satisfy the so-called Silver-Müller radiation condition to the effect that

$$
x \times H+|x| E=o(1) \text { as }|x| \rightarrow \infty .
$$

Likewise, the propagation of an electromagnetic wave inside a confined environment (cavity scattering), such as the region of the space occupied by $\Omega$, is governed by (1.2) with $\Omega_{-}$replaced by $\Omega_{+}$. These are one-sided phenomena. The transmission boundary value problem for the Maxwell system is a two-sided phenomenon, in which one seeks to determine two pairs of vector fields $\left(E^{+}, H^{+}\right)$and $\left(E^{-}, H^{-}\right)$ which satisfy Maxwell's equations on either side of an interface, thought of as the boundary of a bounded Lipschitz domain $\Omega \subset \mathbb{R}^{3}$, in which the fields in question interact on the boundary, and the fields in $\Omega_{-}$decay at infinity (in the sense explained above). Hence, in concrete terms, the transmission boundary value problem 
for the Maxwell system reads

$$
\left\{\begin{array}{l}
\operatorname{curl} E^{+}-i k H^{+}=0 \text { in } \Omega_{+}, \\
\operatorname{curl} H^{+}+i k E^{+}=0 \text { in } \Omega_{+}, \\
\operatorname{curl} E^{-}-i k H^{-}=0 \text { in } \Omega_{-}, \\
\operatorname{curl} H^{-}+i k E^{-}=0 \text { in } \Omega_{-}, \\
N\left(E^{ \pm}\right), N\left(H^{ \pm}\right) \in L^{p}(\partial \Omega), \\
\nu \times\left. E^{-}\right|_{\partial \Omega}-\nu \times\left. E^{+}\right|_{\partial \Omega}=f \in L_{t a n}^{p, \text { Div }}(\partial \Omega), \\
\nu \times\left. H^{-}\right|_{\partial \Omega}-\mu \nu \times\left. H^{+}\right|_{\partial \Omega}=g \in L_{t a n}^{p, \text { Div }}(\partial \Omega), \\
E^{-}, H^{-} \text {decay at infinity. }
\end{array}\right.
$$

Some words of explanation clarifying the notation used above are in order here. First, $L^{p}(\partial \Omega)$ is the Lebesgue space of $p$-integrable functions on $\partial \Omega$, while $L_{\text {tan }}^{p \text {, Div }}(\partial \Omega)$ is a suitable Sobolev-like space of vector fields on $\partial \Omega$ (consisting of $L^{p}$ tangential fields whose surface divergence is also in $L^{p}$; see Section 3 for detailed definitions). Second, $\nu$ denotes the outwardly pointing unit normal to $\partial \Omega$, whereas $\mu \in(0,1)$ is a coupling transmission parameter which is determined by the physical characteristics of the media occupying $\Omega_{+}$and $\Omega_{-}$. Third, given a (possibly vector-valued) function $u$ defined in $\Omega$, its non-tangential maximal function $N u$ is defined as

$$
(N u)(x):=\sup \{|u(y)|: y \in \Omega,|x-y| \leq \kappa \operatorname{dist}(y, \partial \Omega)\}, \quad x \in \partial \Omega,
$$

where $\kappa>1$ is some fixed constant. Furthermore, the non-tangential boundary trace of $u$ on $\partial \Omega$ is taken to be

$$
\left.u\right|_{\partial \Omega}(x):=\lim _{\substack{\Omega \ni y \rightarrow x \\|x-y| \leq \kappa \operatorname{dist}(y, \partial \Omega)}} u(y), \quad x \in \partial \Omega,
$$

whenever the limit exists. Similar interpretation (vis a vis to (1.5) and (1.6)) apply in the case when $u$ is defined in $\Omega_{-}$. Returning for a moment to (1.4), we wish to note that this problem models the scattering of time-harmonic electromagnetic waves by a penetrable bounded obstacle $\Omega$ (see, e.g., [CK83, [DL90, GK96]). Such phenomena are extremely common in real life. Examples include light propagating through air and glass, radar waves penetrating composite materials, etc.

A related problem is the transmission boundary value problem for the Helmholtz operator $\Delta+k^{2}$, in which the task is to find two scalar-valued functions $w^{ \pm}$which satisfy

$$
\left\{\begin{array}{l}
\left(\Delta+k^{2}\right) w^{ \pm}=0 \text { in } \Omega_{ \pm}, \\
N\left(\nabla w^{ \pm}\right), N\left(w^{ \pm}\right) \in L^{p}(\partial \Omega), \\
\left.w^{+}\right|_{\partial \Omega}-\left.\mu w^{-}\right|_{\partial \Omega}=\phi \in L_{1}^{p}(\partial \Omega), \\
\partial_{\nu} w^{+}-\partial_{\nu} w^{-}=\psi \in L^{p}(\partial \Omega), \\
w^{-} \text {decays at infinity, }
\end{array}\right.
$$


where the last condition above means that $w^{-}$decays exponentially if $\operatorname{Im} k>0$, and $w^{-}$satisfies the so-called Sommerfeld radiation condition, i.e.,

$$
\lim _{|x| \rightarrow \infty}\left\{\left\langle x,\left(\nabla w^{-}\right)(x)\right\rangle-i k|x| w^{-}(x)\right\}=0,
$$

in the case when $k \in \mathbb{R} \backslash\{0\}$. Above, $L_{1}^{p}(\partial \Omega)$ stands for the $L^{p}$-based Sobolev space of order one on $\partial \Omega$, and $\langle\cdot, \cdot\rangle$ denotes the standard inner-product in $\mathbb{R}^{3}$. The problem (1.7) models the propagation of acoustic waves penetrating through two media which exhibit different physical characteristics.

The existing literature on the Maxwell and Helmholtz boundary value problems views them as related, yet distinct, problems requiring separate treatments. However, the various (seemingly miraculous) similarities and connections between these problems can be explained in a most satisfactory manner through the language of Clifford analysis. Indeed, it has long been understood that there are basic connections between the Maxwell system and the Helmholtz operator with wave number $k$, on the one hand, and a certain perturbed Dirac operator $\mathbb{D}_{k}$, on the other hand, which we would now like to describe. Consider the Clifford algebra $\mathrm{Cl}_{4}$ freely generated by four anti-commuting imaginary units $e_{1}, e_{2}, e_{3}, e_{4}$ and, given a wave number $k \in \mathbb{C}$, define the perturbed Dirac operator

$$
\mathbb{D}_{k}:=e_{1} \partial_{1}+e_{2} \partial_{2}+e_{3} \partial_{3}+k e_{4},
$$

naturally acting (from the left) on $\mathrm{Cl}_{4}$-valued functions. The key observation which, in essence, goes back to Maxwell himself but which has been best formalized by K. Clifford and M. Riesz, is that the Maxwell system can be written as a single equation which expresses the Clifford analyticity of a certain $\mathrm{Cl}_{4}$-valued function. Specifically, if $E$ and $H$ are the electric and magnetic components of an electromagnetic wave in $\mathbb{R}^{3}$, then for $k \neq 0$ the entire Maxwell system (1.2) is equivalent to the Maxwell-Dirac equation

$$
\mathbb{D}_{k}\left(H-i e_{4} \odot E\right)=0 \text { in } \Omega_{-} .
$$

In (1.10), $\odot$ denotes the operation of multiplication in $\mathrm{Cl}_{4}$ and the vector fields $E=\left(E_{1}, E_{2}, E_{3}\right)$ and $H=\left(H_{1}, H_{2}, H_{3}\right)$ are regarded as Clifford algebra-valued functions via the natural (vector and bi-vector, respectively) identifications

$$
\begin{gathered}
E=E_{1} e_{1}+E_{2} e_{2}+E_{3} e_{3} \quad \text { and } \\
H=H_{1} e_{2} \odot e_{3}+H_{2} e_{3} \odot e_{1}+H_{3} e_{1} \odot e_{2} .
\end{gathered}
$$

On the other hand, the close relationship between the Helmholtz operator and the perturbed Dirac operator is apparent from the factorization formula

$$
\mathbb{D}_{k}^{2}=-\left(\Delta+k^{2}\right),
$$

which shows that $w$ is a null-solution of $\Delta+k^{2}$ if and only if $u:=\mathbb{D}_{k} w$ is a null-solution of $\mathbb{D}_{k}$.

Motivated by (1.10) and (1.12), the goal of this paper is to set up and solve transmission boundary value problems for the perturbed Dirac operator $\mathbb{D}_{k}$ and then, a posteriori, find ways to extract information about the Maxwell and Helmholtz transmission problems (1.4) and (1.7) as corollaries of this more general analysis. Succinctly put, our goal is to exploit the connections highlighted above and present a coherent, unified approach to transmission problems which relies on the Clifford algebra formalism. 
The main result of our work is summarized in the theorem below. To state it, we let 'wedge' and 'backward wedge' denote, respectively, the exterior and interior products. For example, we have $e_{j} \wedge e_{k}=\left(1-\delta_{j k}\right) e_{j} \odot e_{k}$ and $e_{j} \vee e_{k}=-\delta_{j k} e_{j} \odot e_{k}$ (cf. Section 2 for a more detailed exposition). Also, we let $u_{\text {tan }}$ and $u_{n o r}$ stand, respectively, for the tangential and normal components of a $\mathrm{Cl}_{4}$-valued function $u$ defined on $\partial \Omega$. Furthermore, as before, $\nu=\left(\nu_{1}, \nu_{2}, \nu_{3}\right)$ denotes the outward unit normal to $\Omega$, and we identify it with the Clifford algebra-valued function $\nu=\nu_{1} e_{1}+\nu_{2} e_{2}+\nu_{3} e_{3}$. Finally, we let $d$ and $\delta$ stand for the exterior derivative operator and its formal adjoint, respectively (in a differential geometric sense). Here is the theorem which constitutes the main result of this paper.

Theorem 1.1. Given a bounded Lipschitz domain $\Omega$ in $\mathbb{R}^{3}, 1<p<\infty, k \in \mathbb{C} \backslash\{0\}$ with $\operatorname{Im} k \geq 0$, and $\mu \in(0,1)$, consider the following transmission boundary value problem for the perturbed Dirac operator $\mathbb{D}_{k}$ :

$$
\left\{\begin{array}{l}
u^{ \pm} \in C^{1}\left(\Omega_{ \pm}, \mathcal{C}_{4}\right), \\
\mathbb{D}_{k} u^{ \pm}=0 \text { in } \Omega_{ \pm}, \\
u_{\text {nor }}^{+}-u_{\text {nor }}^{-}=f \in L^{p}\left(\partial \Omega, \mathcal{C}_{4}\right), \\
u_{\text {tan }}^{+}-\mu u_{\text {tan }}^{-}=g \in L^{p}\left(\partial \Omega, C_{4}\right), \\
u^{-} \text {decays at infinity, } \\
N\left(u^{ \pm}\right), N\left(d u^{ \pm}\right) \text {and } N\left(\delta u^{ \pm}\right) \in L^{p}(\partial \Omega),
\end{array}\right.
$$

where all boundary traces are taken in the pointwise non-tangential limit sense (cf. (1.6) ).

Then there exist $\varepsilon>0$ and an at most countable set of real numbers $\left\{k_{j}\right\}_{j}$ which depend exclusively on the boundary $\partial \Omega$ and which have the following significance. Whenever $p \in(1,2+\varepsilon)$ and $k \in \mathbb{C} \backslash\left\{k_{j}\right\}_{j}$ has $\operatorname{Im} k \geq 0$, the Dirac transmission problem (1.13) has a solution if and only if

$$
f \in \nu \wedge L_{\text {tan }}^{p, \delta}\left(\partial \Omega, \mathcal{C l}_{4}\right) \text { and } g \in \nu \vee L_{\text {nor }}^{p, d}\left(\partial \Omega, \mathcal{C l}_{4}\right)
$$

Furthermore, the solution $\left(u^{+}, u^{-}\right)$is unique and, granted (1.14), satisfies the estimate

$$
\begin{aligned}
\left\|N\left(u^{ \pm}\right)\right\|_{L^{p}(\partial \Omega)}+\left\|N\left(d u^{ \pm}\right)\right\|_{L^{p}(\partial \Omega)}+\left\|N\left(\delta u^{ \pm}\right)\right\|_{L^{p}(\partial \Omega)} \\
\leq C\left(\|\nu \vee f\|_{L_{\text {tan }}^{p, \delta}\left(\partial \Omega, C_{4}\right)}+\|\nu \wedge g\|_{L_{\text {nor }}^{p, d}\left(\partial \Omega, C_{4}\right)}\right)
\end{aligned}
$$

where $C>0$ depends only on $\partial \Omega, \mu, k, p$, and it can be represented in terms of integral operators acting on the boundary data.

This result is proved in (the last part of) Section 7 , where a slightly different formulation is adopted (cf. Theorem 7.1). The spaces appearing in (1.14) are defined in Section 3, on which occasion a detailed analysis of their properties is carried out. Here we only want to point out that an equivalent reformulation of (1.15) involving only norms in standard Lebesgue spaces reads as follows: For any Clifford algebra-valued functions $u^{ \pm}$defined in $\Omega_{ \pm}$, satisfying $\mathbb{D}_{k} u^{ \pm}=0$ in $\Omega_{ \pm}$, which satisfy $N\left(u^{ \pm}\right), N\left(d u^{ \pm}\right), N\left(\delta u^{ \pm}\right) \in L^{p}(\partial \Omega)$, and for which $u^{-}$decays at 
infinity, there holds

$$
\begin{aligned}
& \left\|N\left(u^{ \pm}\right)\right\|_{L^{p}(\partial \Omega)}+\left\|N\left(d u^{ \pm}\right)\right\|_{L^{p}(\partial \Omega)}+\left\|N\left(\delta u^{ \pm}\right)\right\|_{L^{p}(\partial \Omega)} \\
& \quad \leq C\left\|u_{\text {nor }}^{+}-u_{n o r}^{-}\right\|_{L^{p}(\partial \Omega)}+C\left\|\left(\delta u^{+}\right)_{n o r}-\left(\delta u^{-}\right)_{n o r}\right\|_{L^{p}(\partial \Omega)} \\
& \quad+C\left\|u_{\text {tan }}^{+}-\mu u_{\text {tan }}^{-}\right\|_{L^{p}(\partial \Omega)}+C\left\|\left(d u^{+}\right)_{t a n}-\mu\left(d u^{-}\right)_{t a n}\right\|_{L^{p}(\partial \Omega)}
\end{aligned}
$$

whenever $1<p<2+\varepsilon$, where $C=C(\partial \Omega, k, p, \mu)>0$ is independent of $u^{ \pm}$.

Based on our experience with other similar boundary value problems we conjecture that the condition $1<p<2+\varepsilon$ guaranteeing the above well-posedness result is sharp in the class of three-dimensional Lipschitz domains. Although we do not explicitly pursue this in this paper, by combining the results in HMT10. with the techniques developed here it is possible to show that the problem (1.13) is well-posed for arbitrary data $f, g$ as in (1.14) for a given $p \in(1, \infty)$ provided the outward unit normal has infinitesimal mean oscillations which are sufficiently small relative to the given exponent $p$. More precisely, one demands that

$$
\lim _{\varepsilon \rightarrow 0^{+}}\left(\sup _{\substack{0<r<\varepsilon \\ z \in \partial \Omega}} f_{B(z, r) \cap \partial \Omega} f_{B(z, r) \cap \partial \Omega}|\nu(x)-\nu(y)| d \sigma(x) d \sigma(y)\right)
$$

is appropriately small, depending on the chosen $p \in(1, \infty)$. As a consequence, given a bounded Lipschitz domain whose unit normal belongs to the Sarason space of functions of vanishing mean oscillations on $\partial \Omega$, the Dirac transmission problem (1.13) is well-posed for any $p \in(1, \infty)$ if the unit normal to $\Omega$ has vanishing mean oscillations. In particular, this is the case when $\partial \Omega \in C^{1}$. The interested reader is also referred to $\mathrm{H}-\mathrm{T} 09$ for other criteria (e.g., involving the angle between the two Hardy spaces consisting of boundary traces of inner and outer monogenic functions) ensuring the smallness of (1.17). The above considerations hold in any space dimensions $m \geq 3$. In the higher-dimensional setting, the methods of the current paper yield the well-posedness of the analogue of (1.4) formulated in arbitrary Lipschitz subdomains of $\mathbb{R}^{m}, m \geq 4$, for $p \in(2-\varepsilon, 2+\varepsilon)$.

The fact that we are presently restricting ourselves to the case $m=3$ has its origins in results presented in Section 5 (more precisely, in Steps 3 and 4 in the proof of Lemma 5.4). Here we are exploiting certain intertwining identities between the boundary integral operators which are relevant in the treatment of the Maxwell transmission problem, on the one hand, and the (better understood) scalar potentials for the Helmholtz operator, on the other hand, which are specific to the case when $m=3$.

As already indicated, part of the interest in the Dirac transmission boundary value problem (1.13) stems from the fact that this is intimately connected with the Maxwell and Helmholtz transmission boundary value problems (1.4), (1.7). Ultimately, the present approach can be thought of as an elliptization scheme for the Maxwell system. Indeed, in Section 8 we find necessary and sufficient conditions for the boundary data which guarantee that the Dirac transmission problem (1.13) essentially amounts to four (scalar) Helmholtz transmission problems (cf. (8.45)(8.48)) and two (vector) Maxwell transmission problems (cf. (8.38), (8.39)). In broad terms, the Maxwell system is 'embedded' into a more general, elliptic system 
via a procedure which also identifies the (more specialized) type of boundary data for which the two systems are actually equivalent.

As is implicit in the statement of the above theorem, we rely on the method of boundary singular integral operators of Clifford-Cauchy type, which are defined and systematically treated in Section 4 . This is not unlike the treatment of other systems of elliptic PDE's, such as the Lamé system of elasticity and the Stokes system of hydrodynamics. However, the Dirac transmission problem (1.13) presents unique challenges, due to its highly specialized algebraic nature. This is already apparent from the nature of the space of boundary data (cf. (1.14)) which have been suitably designed to fit the format of the PDE in question (we wish to stress that selecting boundary data as in (1.14) is not merely convenient but, in fact, necessary, as the statement of Theorem 1.1 shows). Also, at a technical level, unlike the case of other systems of PDE's, the range $1<p \leq 2$ is not obtained by interpolating between the limiting cases $p=1$ and $p=2$, as it is unclear what the versions of the spaces appearing in (1.14) are when $p=1$ (in which scenario one would have to work with some kind of Hardy space). Instead, we devise an approach which requires a thorough understanding of the interplay between the magnetic dipole operator $M_{k}$ alluded to before and the better studied double layer potential operator for the Helmholtz operator. See Section 5 for details.

Here is a brief survey of related work. Most of the transmission boundary value problems considered in the literature fall under several categories, depending on the nature of the domain and solution. First, there is the class of problems in domains with sufficiently smooth boundaries (so that they can be flattened and/or pseudo-differential operator techniques - with a limited amount of smoothnesscan be used). See, e.g., LRU66], KP98, KM88, for scalar equations, and Wi87, Rei93, BD54, AK92, MO93, CK83, Mu51, Mu69 for Maxwell's equations. Second, there is the class of problems in domains with isolated singularities, such as those treated in KMR01, Re89a, Re89b, [NS99]. Weak (variational) solutions for transmission problems in Lipschitz domains are discussed in, e.g., Sa52, Ag97. Finally, strong solutions in Dahlberg's sense (cf. Da77) for transmission problems in Lipschitz domains are treated in EFV92, ES93, MM98, Seo97, for single equations, and ES93, MM98, for systems of PDE's. Related work for one-sided boundary value problems for Dirac's equation in Lipschitz domains may also be found in McMM97, Mi01, Mi02.

We conclude this section by outlining the plan of the paper. Section 2 is devoted to reviewing Clifford algebras and the formalism associated with Clifford calculus. In Section 3, we introduce and study spaces of functions defined on Lipschitz surfaces which measure smoothness in a way which is compatible with the nature of the perturbed Dirac operator. Section 4 contains a discussion of issues pertaining to the boundary behavior of integral operators of Clifford-Cauchy type on Lipschitz domains. Next, in Section 5 , we study the invertibility of the principal-value version of the aforementioned integral operators on the spaces introduced in Section 3. Subsequently, in Section 6, these operators are used in connection with Hardy spaces and half-Dirichlet problems for the perturbed Dirac operator. Section 7 contains the formulation and proof of the main result in this paper. Finally, in Section 8, we build a (two-way) bridge between the Dirac transmission problem, on the one hand, and the Maxwell and Helmholtz transmission problems, on the other hand. 


\section{Review of Clifford Calculus}

Since the formalism associated with Clifford algebras will play a fundamental role in this paper, here we collect a number of basic results pertaining to Clifford calculus. For more background material and further general references on Clifford algebras and related matters, the interested reader is referred to the monographs BDS82, GM91] and Mi94.

Most of the work in this paper will be carried out in the framework of the (complex) Clifford algebra $\mathcal{C}_{m}$ with $m$ generators, $\left\{e_{j}\right\}_{j=1}^{m}$. The Clifford algebra product will be denoted by $\odot$, which should not be confused with the operation of symmetric multiplication of tensors. In particular, we have the standard anticommutativity relations $e_{i} \odot e_{j}=-e_{j} \odot e_{i}$ for any $i \neq j$, and $e_{j}^{2}=-1$. Recall that elements in $\mathcal{C}_{m}$ may be uniquely written in the form $u=\sum_{\ell=0}^{m} \sum_{|I|=\ell}^{\prime} u_{I} e_{I}$ with $u_{I} \in \mathbb{C}$, where $e_{I}$ stands for the product $e_{i_{1}} \odot e_{i_{2}} \odot \cdots \odot e_{i_{\ell}}$ if $I=\left(i_{1}, i_{2}, \ldots, i_{\ell}\right)$ with $1 \leq i_{1}<i_{2}<\cdots<i_{\ell} \leq m$. Here we agree that $e_{0}:=e_{\emptyset}:=1$, and that $\sum_{I}^{\prime}$ indicates that the sum is performed over (strictly) increasingly ordered $n$-tuples $I$. In particular, we have the natural embedding $\mathbb{R}^{m} \hookrightarrow \mathcal{C}_{m}$ given by $\mathbb{R}^{m} \ni x=\left(x_{j}\right)_{1 \leq j \leq m} \equiv \sum_{j=1}^{m} x_{j} e_{j} \in \mathcal{C}_{m}$.

The Clifford conjugation on $\mathcal{C} \ell_{m}$, denoted by 'bar', is defined as the unique complex-linear involution on $\mathcal{C l}_{m}$ for which $\overline{e_{I}} \odot e_{I}=e_{I} \odot \overline{e_{I}}=1$ for any multiindex $I$. In particular, given $u=\sum_{I}^{\prime} u_{I} e_{I} \in \mathcal{C} \ell_{m}$, then $\bar{u}=\sum_{I}^{\prime} u_{I} \overline{e_{I}}$. We will also use the complex Clifford conjugation on $\mathcal{C}_{m}$ defined for $u=\sum_{I}^{\prime} u_{I} e_{I}$ as $u^{c}:=\sum_{I}^{\prime} u_{I}^{c} e_{I}$, where $a^{c}$ denotes the usual complex conjugation of $a \in \mathbb{C}$. Next, define the scalar part of $u=\sum_{I}^{\prime} u_{I} e_{I} \in \mathcal{C}_{m}$ as $u_{0}:=u_{\emptyset}$, and endow $\mathcal{C}_{m}$ with the bilinear form

$$
\langle u, v\rangle:=\sum_{I}^{\prime} u_{I} v_{I}, \quad \text { if } u=\sum_{I}^{\prime} u_{I} e_{I}, \quad v=\sum_{I}^{\prime} v_{I} e_{I} \in \mathcal{C} \ell_{m} .
$$

Note that $\langle u, v\rangle=(u \odot \bar{v})_{0}$ and we define $|u|:=\sqrt{\left\langle u, u^{c}\right\rangle}$ for every $u \in \mathcal{C} \ell_{m}$.

To proceed, for each $\ell \in\{0,1, \ldots, m\}$ consider the projection map $\Pi_{\ell}$ onto the $\ell$-homogeneous part of $u$, i.e.,

$$
\Pi_{\ell} u:=\sum_{|I|=\ell}^{\prime} u_{I} e_{I},
$$

and denote by $\Lambda^{\ell}$ the range of $\Pi_{\ell}: \mathcal{C} \ell_{m} \rightarrow \mathcal{C}_{m}$. It follows that

$$
\mathcal{C}_{m}=\Lambda^{0} \oplus \Lambda^{1} \oplus \cdots \oplus \Lambda^{m} .
$$

Elements in $\Lambda^{0}, \Lambda^{1}$ and $\Lambda^{2}$ are going to be referred to as scalars, vectors and bi-vectors, respectively. As is apparent from definitions, multiplication of a homogeneous Clifford element by a vector consists of two parts in which the degree of homogeneity is either increased or decreased by one unit, so if $a \in \Lambda^{1}$ and $u \in \Lambda^{j}$, then

$$
a \odot u \in \Lambda^{j-1} \oplus \Lambda^{j+1} .
$$

Define the exterior product $\wedge$ and the interior product $\vee$ with an arbitrary, fixed vector $a \in \Lambda^{1}$ by

$$
\begin{gathered}
a \wedge u:=\Pi_{j+1}(a \odot u) \quad \text { and } \quad a \vee u:=-\Pi_{j-1}(a \odot u) \\
\text { for every } u \in \Lambda^{j} .
\end{gathered}
$$


Then extend linearly $\wedge$ and $\vee$ to all $u \in \mathcal{C} \ell_{m}$. As a result, we have

$$
a \odot u=a \wedge u-a \vee u, \quad \text { for any } \quad u \in \mathcal{C}_{m} .
$$

Let us also point out here the (simple but useful) fact that if $a$ is a vector and $u$ is a scalar, then $a \wedge u=u a$ and $a \vee u=0$, whereas if both $a$ and $u$ are vectors, then $a \vee u=\langle a, u\rangle$.

The Hodge star operator can be defined as the unique linear mapping

$$
*: \Lambda^{\ell} \rightarrow \Lambda^{m-\ell}
$$

such that

$$
e_{I} \odot\left(* e_{I}\right)=e_{1} \odot e_{2} \odot \cdots \odot e_{m}
$$

for every multi-index $I=\left(i_{1}, i_{2}, \ldots, i_{m}\right)$ with $|I|=\ell$ and all $\ell \in\{0,1, \ldots, m\}$.

The properties of Clifford calculus which are most relevant for our purposes are collected in the lemma below.

Lemma 2.1. Suppose that $a, b \in \Lambda^{1}$ and that $u \in \Lambda^{\ell}, v \in \Lambda^{m-\ell}$ for some index $\ell \in\{0,1, \ldots, m\}$. Then the following identities hold:

(1) $a \wedge(a \wedge u)=0$ and $a \vee(a \vee u)=0$

(2) $* * u=(-1)^{\ell(m-\ell)} u$.

(3) $\langle u, * v\rangle=(-1)^{\ell(m-\ell)}\langle * u, v\rangle$.

(4) $*(a \wedge u)=(-1)^{\ell} a \vee(* u)$.

(5) $*(a \vee u)=(-1)^{\ell-1} a \wedge(* u)$.

(6) $a \wedge(b \vee u)+b \vee(a \wedge u)=\langle a, b\rangle u$.

(7) $\langle a \wedge u, v\rangle=\langle u, a \vee v\rangle$.

Corollary 2.2. For each $a \in \Lambda^{1}$ with $|a|=1$, and each $u \in \mathcal{C}_{m}$,

$$
u=a \wedge(a \vee u)+a \vee(a \wedge u) .
$$

Let $\Omega$ be a Lipschitz domain with compact boundary in $\mathbb{R}^{m}$ which means (cf. St70, p. 189]) that there exists a finite open covering $\left\{\mathcal{O}_{j}\right\}_{1 \leq j \leq N}$ of $\partial \Omega$ with the property that, for every $j \in\{1, \ldots, N\}, \mathcal{O}_{j} \cap \Omega$ coincides with the portion of $\mathcal{O}_{j}$ lying in the over-graph of a Lipschitz function $\varphi_{j}: \mathbb{R}^{m-1} \rightarrow \mathbb{R}$ (where $\mathbb{R}^{m-1} \times \mathbb{R}$ is a new system of coordinates obtained from the original one via a rigid motion). Such domains are called minimally smooth in E. Stein's book St70. It is a classical result that, for a Lipschitz domain $\Omega$, the surface measure $d \sigma$ is well-defined on $\partial \Omega$ and an outward pointing normal vector $\nu$ exists a.e. on $\partial \Omega$.

In what follows, if $\Omega$ is a Lipschitz domain in $\mathbb{R}^{m}$ we shall identify its unit normal $\nu=\left(\nu_{1}, \ldots, \nu_{m}\right)$ with the Clifford vector $\nu:=\sum_{\ell=1}^{m} \nu_{\ell} e_{\ell}$. Moreover, if $f: \partial \Omega \rightarrow \mathcal{C}_{m}$ is a given Clifford algebra-valued function defined on $\partial \Omega$, introduce its normal and tangential components by setting

$$
f_{\text {nor }}:=\nu \wedge(\nu \vee f) \quad \text { and } \quad f_{\text {tan }}:=\nu \vee(\nu \wedge f)
$$

a.e. on $\partial \Omega$. By Corollary 2.2, $f=f_{\text {nor }}+f_{\text {tan }}$ a.e. on $\partial \Omega$ and, by Lemma 2.1] $\left\langle f_{\text {tan }}, f_{\text {nor }}\right\rangle=0$ and

$$
\nu \wedge f=\nu \wedge f_{\text {tan }} \text { and } \nu \vee f=\nu \vee f_{\text {nor }} .
$$

Moving on, next recall the Dirac operator associated with $\mathcal{C}_{m}$, namely

$$
\mathbb{D}:=\sum_{j=1}^{m} e_{j} \odot \partial_{j} .
$$


Also, recall that a Clifford algebra-valued function $u$, whose components are of class $C^{1}$ in an open subset of $\mathbb{R}^{m}$, is called monogenic if $\mathbb{D} u:=\sum_{j=1}^{m} e_{j} \odot\left(\partial_{j} u\right)=0$.

For reasons which will become apparent later, we find it useful to embed $\mathcal{C}_{m}$ into a larger algebra, $\mathcal{C}_{m+1}$, which has an extra imaginary unit, $e_{m+1}$, compared to $C_{m}$. Hence, throughout the paper, we shall consider the natural embeddings

$$
\mathbb{R}^{m} \hookrightarrow \mathcal{C}_{m} \hookrightarrow \mathcal{C}_{m+1}
$$

Furthermore, in the larger setting of $\mathrm{Cl}_{m+1}$, we shall work with a perturbed Dirac operator, described as follows. Given $k \in \mathbb{C}$, we define $\mathbb{D}_{k}:=\mathbb{D}+k e_{m+1}$. Hence, if $u$ is a $\mathrm{Cl}_{m+1^{-}}$-valued function with $C^{1}$ components, then

$$
\mathbb{D}_{k} u:=\sum_{j=1}^{m} e_{j} \odot \partial_{j} u+k e_{m+1} \odot u,
$$

and we call $u k$-monogenic provided $\mathbb{D}_{k} u=0$. One of the most fundamental properties of Dirac operators introduced above is that they can be thought of as square-roots of familiar second-order differential operators. More precisely, $\mathbb{D}$ and $\mathbb{D}_{k}$ satisfy

$$
\mathbb{D}^{2}=-\Delta \quad \text { and } \quad \mathbb{D}_{k}^{2}=-\left(\Delta+k^{2}\right),
$$

where $\Delta:=\sum_{j=1}^{m} \partial_{j}^{2}$ is the Laplace operator in $\mathbb{R}^{m}$. In particular, $k$-monogenic functions are null-solutions of the Helmholtz operator $\Delta+k^{2}$.

One convenient way to introduce the exterior and interior derivative operators $d, \delta$ is to set

$$
d u:=\Pi_{\ell+1}(\mathbb{D} u), \quad \delta u:=\Pi_{\ell-1}(\mathbb{D} u)
$$

whenever $u$ is a $\Lambda^{\ell}$-valued function with $C^{1}$ components and $0 \leq \ell \leq m$. Hereafter, we shall actually regard $d, \delta$ as operators acting on arbitrary $\mathcal{C}_{m}$-valued functions with $C^{1}$ components by naturally extending $(2.16)$ by linearity to this larger class of functions (cf. also (2.3) in this regard). A simple but useful observation is that for any $C^{1}$ scalar-valued function $\varphi$ one has $d \varphi=\nabla \varphi$ and $\delta \varphi=0$. In addition, $d$ maps any $\Lambda^{m}$-valued function into 0 . From the definitions, it is also straightforward to check that

$$
\mathbb{D}=d+\delta, \quad d^{2}=0, \quad \delta^{2}=0 \quad \text { and } d \delta+\delta d=-\Delta .
$$

We shall frequently use the fact that the Hodge star operator intertwines $d$ and $\delta$. This is made precise in the lemma below.

Lemma 2.3. Suppose that $u$ is a $\Lambda^{\ell}$-valued function, $0 \leq \ell \leq m$, with $C^{1}$ components. Then the following hold:

(i) $* \delta u=(-1)^{\ell} d(* u)$;

(ii) $\delta(* u)=(-1)^{\ell+1} *(d u)$;

(iii) $\delta u=(-1)^{m(\ell+1)+1} *(d(* u))$.

Recall the definitions made in (1.5) and (1.6). The following integration by parts formulas (cf. [MMT01) are going to be useful for us.

Lemma 2.4. Let $\Omega$ be a Lipschitz domain in $\mathbb{R}^{m}$ with compact boundary $\partial \Omega$, and denote by $\sigma$ and $\nu$ its surface measure and outward unit normal, respectively. 
Consider $u, w \in C^{1}\left(\Omega, \mathcal{C} \ell_{m}\right)$ such that

$$
\begin{gathered}
N(|u||w|) \in L^{1}(\partial \Omega), \quad|d u||w|+|u||\delta w| \in L^{1}(\Omega) \\
\text { and }\left.u\right|_{\partial \Omega},\left.\quad w\right|_{\partial \Omega} \text { exist a.e. on } \partial \Omega .
\end{gathered}
$$

In addition, assume that $u, v$ decay at infinity in the case when $\Omega$ is unbounded. Then the following formula holds:

$$
\int_{\Omega}\langle d u, w\rangle d x=\int_{\Omega}\langle u, \delta w\rangle d x+\int_{\partial \Omega}\langle\nu \wedge u, w\rangle d \sigma .
$$

For the remainder of this section we shall entirely restrict our attention to the physically most relevant case, namely $m=3$.

Important Convention. For the remainder of this work, we shall denote by $\Lambda^{0}, \Lambda^{1}, \Lambda^{2}$ and $\Lambda^{3}$, respectively, the scalars, vectors, bi-vectors and tri-vectors associated with the Clifford algebra $\mathrm{Cl}_{3}$ which, in turn, will always be regarded as a sub-algebra of $\mathrm{Cl}_{4}$. In particular, the following structural formula holds:

$$
\mathrm{Cl}_{4}=\mathrm{Cl}_{3} \oplus\left(e_{4} \odot \mathcal{C l}_{3}\right) \text {. }
$$

Hence,

$$
\begin{aligned}
\mathcal{C l}_{4}= & \Lambda^{0} \oplus\left(\Lambda^{1} \oplus e_{4} \odot \Lambda^{0}\right) \oplus\left(\Lambda^{2} \oplus e_{4} \odot \Lambda^{1}\right) \\
& \oplus\left(\Lambda^{3}+e_{4} \odot \Lambda^{2}\right) \oplus\left(\Lambda^{3} \oplus e_{4} \odot \Lambda^{2}\right) \oplus e_{4} \odot \Lambda^{3} .
\end{aligned}
$$

Note that functions originally defined in a domain in $\mathbb{R}^{3}$ and with values in $\mathcal{C l}_{3}$ can be canonically viewed as being $\mathrm{Cl}_{4}$-valued. However, we wish to stress that we will always consider the operators $d, \delta$ and $*$ as being associated with and acting on $\mathrm{C}_{3}$. The task of interchanging the operators $d, \delta$, * on the one hand, and multiplication by the imaginary unit $e_{4}$ on the other hand, is taken up below.

Lemma 2.5. For the operators $d, \delta$ and $*$ acting on a function $u$ with $C^{1}$ components defined in a domain in $\mathbb{R}^{3}$, the following identities hold:

$$
\begin{gathered}
*\left(e_{4} \odot u\right)=e_{4} \odot(* u), \quad d\left(e_{4} \odot u\right)=-e_{4} \odot d u \\
\text { and } \delta\left(e_{4} \odot u\right)=-e_{4} \odot \delta u .
\end{gathered}
$$

This can be verified either directly from definitions, or with the help of the following lemma which will also be important in subsequent developments.

Lemma 2.6. Assume that $a \in \Lambda^{1} \equiv \mathbb{R}^{3}$ and that $u \in \mathcal{C l}_{4}$. Then

$$
a \wedge\left(e_{4} \odot u\right)=-e_{4} \odot(a \wedge u) \quad \text { and } \quad a \vee\left(e_{4} \odot u\right)=-e_{4} \odot(a \vee u) \text {. }
$$

Proof. By linearity, it suffices to first treat the cases $u \in \Lambda^{j} \subset \mathcal{C l}_{3}, 0 \leq j \leq 3$, and then the case $u=e_{4} \odot v$, where $v \in \mathcal{C}_{3}$. Since, in general, $\Pi_{j+1}\left(e_{4} \odot w\right)=e_{4} \odot \Pi_{j}(w)$ if $w \in \mathcal{C l}_{3}, 0 \leq j \leq 3$, the desired conclusion follows readily from the definitions.

The point of the next lemma (whose simple proof is omitted) is to relate standard notation employed in three dimensions to operators introduced earlier in this section.

Lemma 2.7. For any vector fields $u, v$ with $C^{1}$ components defined in a domain in $\mathbb{R}^{3}$, one has

$$
\operatorname{div} u=-\delta u, \quad\langle u, v\rangle=u \vee v, \quad \operatorname{curl} u=*(d u), \quad u \times v=*(u \wedge v) .
$$


In the last part of this section we give two examples illustrating how various operators, originally introduced in the setting of Clifford algebras, can be understood in terms of standard vector calculus in the three-dimensional setting. Below, we let $\Omega$ stand for an arbitrary Lipschitz domain in $\mathbb{R}^{3}$ and denote by $\nu$ its outward unit normal.

Example 1. Let $u$ be an arbitrary $C_{3}$-valued function. First, by (2.3), (2.7) and (2) in Lemma 2.1 $u$ can be uniquely written as

$$
u=u_{0}+u_{1}+* u_{1}^{\prime}+* u_{0}^{\prime},
$$

where the functions $u_{j}, u_{j}^{\prime}$ are $\Lambda^{j}$-valued, $j \in\{0,1\}$.

Throughout this work, given an arbitrary $\mathrm{Cl}_{3}$-valued function $u$, we agree to let $u_{0}, u_{1}, u_{0}^{\prime}, u_{1}^{\prime}$ retain the same significance as above, and refer to (2.25) as the canonical decomposition of $u$.

Furthermore, if actually $u$ is defined in $\Omega$ and happens to be differentiable, then the functions $u_{j}, u_{j}^{\prime}$ are also differentiable in $\Omega$. In this scenario, using (2.1), (2.3) and (2.7), we compute

$$
d u=\nabla u_{0}+*\left(\operatorname{curl} u_{1}\right)+*\left(\operatorname{div} u_{1}^{\prime}\right)
$$

and

$$
\delta u=-\operatorname{div} u_{1}+\operatorname{curl} u_{1}^{\prime}-*\left(\nabla u_{0}^{\prime}\right) .
$$

Example 2. Let $f: \partial \Omega \rightarrow \mathcal{C l}_{3}$ be an arbitrary function and consider the canonical decomposition of $f$ given by $f=f_{0}+f_{1}+* f_{1}^{\prime}+* f_{0}^{\prime}$. Using the properties listed in Lemma 2.1 we then obtain

$$
\nu \wedge f=\nu f_{0}+*\left(\nu \times f_{1}\right)+*\left\langle\nu, f_{1}^{\prime}\right\rangle
$$

and

$$
\nu \vee f=\left\langle\nu, f_{1}\right\rangle-\nu \times f_{1}^{\prime}+*\left(\nu f_{0}^{\prime}\right) .
$$

\section{BOUNDARY SMOOTHNESS SPACES}

Throughout this section we fix a bounded Lipschitz domain $\Omega$ in $\mathbb{R}^{m}$, and denote by $\nu$ and $\sigma$ its outward unit normal and surface measure, respectively. The goal is to introduce and study a number of spaces of functions, well-suited for the type of PDE's we intend to treat later, which are defined on $\partial \Omega$ and take complex, vector or Clifford algebra values, as well as the action of various tangential differential operators on these spaces.

To get started, hereafter we shall let $L^{p}(\partial \Omega), 1 \leq p \leq \infty$, stand for the Lebesgue space of complex-valued, measurable functions which are $p$-th power integrable with respect to $d \sigma$ on $\partial \Omega$. We then define $L_{1}^{p}(\partial \Omega)$ as the first-order, $L^{p}$-based Sobolev space of functions on $\partial \Omega$, i.e., the collection of all functions $f \in L^{p}(\partial \Omega)$ whose tangential gradient, $\nabla_{\tan } f$, has components in $L^{p}(\partial \Omega)$. Recall that if $f$ has a $C^{1}$ extension in a neighborhood of $\partial \Omega$ (which we continue to denote by $f$ ), then $\nabla_{\text {tan }} f=\nabla f-(\nu \cdot \nabla f) \nu$ on $\partial \Omega$. In this vein, it is worth pointing out that, when $m=3$, we have $\nabla_{\text {tan }} f=-\nu \times(\nu \times \nabla f)$. We equip $L_{1}^{p}(\partial \Omega)$ with the natural norm, given by $\|f\|_{L_{1}^{p}(\partial \Omega)}:=\|f\|_{L^{p}(\partial \Omega)}+\left\|\nabla_{\tan } f\right\|_{L^{p}(\partial \Omega)}$. The dual of the Sobolev space just introduced is going to be denoted by $L_{-1}^{p^{\prime}}(\partial \Omega):=\left(L_{1}^{p}(\partial \Omega)\right)^{*}$, where $\frac{1}{p}+\frac{1}{p^{\prime}}=1$. 
Since, as is well known, $L_{1}^{p}(\partial \Omega)$ is reflexive, we also have $L_{1}^{p}(\partial \Omega)=\left(L_{-1}^{p^{\prime}}(\partial \Omega)\right)^{*}$, whenever $\frac{1}{p}+\frac{1}{p^{\prime}}=1$.

We now elaborate on a useful membership criterion to the Sobolev space $L_{1}^{p}(\partial \Omega)$ in the three-dimensional setting, i.e. when $m=3$. To this end, let $\varphi \in C_{0}^{\infty}\left(\mathbb{R}^{3}, \mathbb{R}^{3}\right)$ and assume that $\psi$ is a scalar-valued function of class $C^{1}$ in a neighborhood of $\partial \Omega$. Then

$$
\int_{\partial \Omega}\langle\nu, \operatorname{curl} \varphi\rangle \psi d \sigma=-\int_{\partial \Omega}\langle\nu \times \nabla \psi, \varphi\rangle d \sigma=-\int_{\partial \Omega}\left\langle\nu \times \nabla_{t a n} \psi, \varphi\right\rangle d \sigma
$$

which shows that

$$
\begin{aligned}
\left\|\nabla_{\text {tan }} \psi\right\|_{L^{p}(\partial \Omega)}=\sup \left\{\left|\int_{\partial \Omega}\langle\nu, \operatorname{curl} \varphi\rangle \psi d \sigma\right|:\right. & \\
\varphi & \left.\in C_{0}^{\infty}\left(\mathbb{R}^{3}, \mathbb{R}^{3}\right),\|\varphi\|_{L^{p^{\prime}}\left(\partial \Omega, \mathbb{R}^{3}\right)} \leq 1\right\},
\end{aligned}
$$

where $1 / p+1 / p^{\prime}=1$. As a consequence, for every $f \in L^{p}(\partial \Omega)$ we have (again, with $\left.1 / p+1 / p^{\prime}=1\right)$

$$
\begin{gathered}
f \in L_{1}^{p}(\partial \Omega) \quad \text { if and only if } \\
\sup \left\{\left|\int_{\partial \Omega}\langle\nu, \operatorname{curl} \varphi\rangle f d \sigma\right|: \varphi \in C_{0}^{\infty}\left(\mathbb{R}^{3}, \mathbb{R}^{3}\right),\|\varphi\|_{L^{p^{\prime}}\left(\partial \Omega, \mathbb{R}^{3}\right)} \leq 1\right\}<\infty .
\end{gathered}
$$

Next, we briefly discuss the surface divergence operator. First, if $1<p<\infty$, let $L_{t a n}^{p}(\partial \Omega)$ stand for the space of tangential, complex-valued, vector fields on $\partial \Omega$, with components in $L^{p}(\partial \Omega)$. In other words,

$$
L_{\text {tan }}^{p}(\partial \Omega):=\left\{f \in L^{p}\left(\partial \Omega, \mathbb{C}^{m}\right):\langle\nu, f\rangle=0 \text { a.e. on } \partial \Omega\right\},
$$

which we view as a closed subspace of $L^{p}\left(\partial \Omega, \mathbb{C}^{m}\right)$. Note that the tangential gradient $\nabla_{t a n}: L_{1}^{p}(\partial \Omega) \rightarrow L_{t a n}^{p}(\partial \Omega)$ becomes a well-defined, linear and bounded operator. We then define the surface divergence operator as

$$
\text { Div : } L_{t a n}^{p}(\partial \Omega) \longrightarrow L_{-1}^{p}(\partial \Omega), \quad\langle\operatorname{Div} f, g\rangle:=-\int_{\partial \Omega}\left\langle f, \nabla_{t a n} g\right\rangle d \sigma
$$

for each $f \in L_{t a n}^{p}(\partial \Omega)$ and $g \in L_{1}^{p^{\prime}}(\partial \Omega)=\left(L_{-1}^{p}(\partial \Omega)\right)^{*}$, with $\frac{1}{p}+\frac{1}{p^{\prime}}=1$. Above we made use of the convention that the symbol $\langle\cdot, \cdot\rangle$ is also employed to denote the natural duality pairing between a Banach space and its dual. One way of understanding (3.5) is to say that the surface divergence operator is the adjoint of $-\nabla_{\text {tan }}$

Another space important for us in the sequel is

$$
L_{\text {tan }}^{p, \text { Div }}(\partial \Omega):=\left\{f \in L_{\text {tan }}^{p}(\partial \Omega): \operatorname{Div} f \in L^{p}(\partial \Omega)\right\},
$$

which, when equipped with the norm $\|f\|_{L_{\text {tan }}^{p, \text { Div }}(\partial \Omega)}:=\|f\|_{L^{p}(\partial \Omega)}+\|\operatorname{Div} f\|_{L^{p}(\partial \Omega)}$, becomes a Banach space. We will also need the following closed subspace of (3.6):

$$
L_{\text {tan }}^{p, 0}(\partial \Omega):=\left\{f \in L_{\text {tan }}^{p, \text { Div }}(\partial \Omega): \operatorname{Div} f=0 \text { on } \partial \Omega\right\} .
$$

Moving on, we now discuss spaces of $\mathcal{C}_{m}$-valued functions on $\partial \Omega$. First, for a fixed $p \in(1, \infty)$ we set $L^{p}\left(\partial \Omega, \mathcal{C} \ell_{m}\right):=L^{p}(\partial \Omega) \otimes \mathcal{C}_{m}$. Next, we consider the closed subspace of $L^{p}\left(\partial \Omega, \mathcal{C} \ell_{m}\right)$ consisting of normal functions, i.e., functions $f$ satisfying $\nu \wedge f=0$ a.e. on $\partial \Omega$. Thus,

$$
L_{n o r}^{p}\left(\partial \Omega, \mathcal{C}_{m}\right):=\left\{f \in L^{p}\left(\partial \Omega, \mathcal{C}_{m}\right): \nu \wedge f=0 \text { a.e. on } \partial \Omega\right\} .
$$


Similarly, we introduce the closed subspace of $L^{p}\left(\partial \Omega, \mathcal{C}_{m}\right)$ consisting of tangential functions, i.e., functions $f$ satisfying $\nu \vee f=0$ a.e. on $\partial \Omega$. In other words,

$$
L_{\text {tan }}^{p}\left(\partial \Omega, \mathcal{C}_{m}\right):=\left\{f \in L^{p}\left(\partial \Omega, \mathcal{C}_{m}\right): \nu \vee f=0 \text { a.e. on } \partial \Omega\right\} .
$$

Next, we introduce certain first-order tangential derivative operators along with smoothness spaces which allow for realizations of these operators as bounded, linear mappings between Banach spaces. First, $f \in L_{n o r}^{p}\left(\partial \Omega, C_{m}\right)$ is said to have its boundary exterior derivative $d_{\partial}$ in $L^{p}\left(\partial \Omega, \mathcal{C}_{m}\right)$ if there exists a function in $L^{p}\left(\partial \Omega, \mathcal{C}_{m}\right)$, which we will denote by $d_{\partial} f$, such that

$$
\int_{\partial \Omega}\left\langle d_{\partial} f, \varphi\right\rangle d \sigma=\int_{\partial \Omega}\langle f, \delta \varphi\rangle d \sigma, \forall \varphi \in C_{0}^{\infty}\left(\mathbb{R}^{m}, \mathcal{C} \ell_{m}\right):=C_{0}^{\infty}\left(\mathbb{R}^{m}\right) \otimes \mathcal{C} \ell_{m} .
$$

In connection with this, we then define the space

$$
L_{\text {nor }}^{p, d}\left(\partial \Omega, \mathcal{C}_{m}\right):=\left\{f \in L_{\text {nor }}^{p}\left(\partial \Omega, \mathcal{C l}_{m}\right): d_{\partial} f \in L^{p}\left(\partial \Omega, \mathcal{C}_{m}\right)\right\},
$$

equipped with the norm $\|f\|_{L_{n o r}^{p, d}\left(\partial \Omega, C_{m}\right)}:=\|f\|_{L^{p}\left(\partial \Omega, \ell_{m}\right)}+\left\|d_{\partial} f\right\|_{L^{p}\left(\partial \Omega, \ell_{m}\right)}$.

Similarly, a function $f \in L_{\text {tan }}^{p}\left(\partial \Omega, \mathcal{C l}_{m}\right)$ is said to have its boundary interior derivative $\delta_{\partial}$ in $L^{p}\left(\partial \Omega, \mathcal{C} \ell_{m}\right)$ provided there exists a function in $L^{p}\left(\partial \Omega, \mathcal{C} \ell_{m}\right)$, which we denote by $\delta_{\partial} f$, such that

$$
\int_{\partial \Omega}\left\langle\delta_{\partial} f, \varphi\right\rangle d \sigma=\int_{\partial \Omega}\langle f, d \varphi\rangle d \sigma, \quad \forall \varphi \in C_{0}^{\infty}\left(\mathbb{R}^{m}, \mathcal{C l}_{m}\right) .
$$

We then introduce the space

$$
L_{t a n}^{p, \delta}\left(\partial \Omega, \mathcal{C}_{m}\right):=\left\{f \in L_{t a n}^{p}\left(\partial \Omega, \mathcal{C}_{m}\right): \delta_{\partial} f \in L^{p}\left(\partial \Omega, \mathcal{C}_{m}\right)\right\}
$$

and equip it with the norm $\|f\|_{L_{t a n}^{p, \delta}\left(\partial \Omega, C_{m}\right)}:=\|f\|_{L^{p}\left(\partial \Omega, C_{m}\right)}+\left\|\delta_{\partial} f\right\|_{L^{p}\left(\partial \Omega, C_{m}\right)}$.

Some basic properties of the operators $d_{\partial}$ and $\delta_{\partial}$ are recorded in the two lemmas below, which are closely related to results from [MMT01].

Lemma 3.1. One has $\delta_{\partial} \circ \delta_{\partial}=0$ in the sense that for every $f \in L_{\text {tan }}^{p, \delta}\left(\partial \Omega, \mathcal{C}_{m}\right)$ it follows that $\delta_{\partial} f \in L_{\text {tan }}^{p, \delta}\left(\partial \Omega, \mathcal{C}_{m}\right)$ and $\delta_{\partial}\left(\delta_{\partial} f\right)=0$. Furthermore, $d_{\partial} \circ d_{\partial}=0$ with a similar interpretation.

Lemma 3.2. Let $u \in C^{1}\left(\Omega, C_{m}\right)$ be a function with the property that $u$ and $d u$ have non-tangential boundary traces a.e. on $\partial \Omega$ and $N(u), N(d u) \in L^{p}(\partial \Omega)$. Then $\left.\nu \wedge u\right|_{\partial \Omega} \in L_{\text {nor }}^{p, d}\left(\partial \Omega, \mathrm{Cl}_{m}\right)$ and

$$
d_{\partial}\left(\left.\nu \wedge u\right|_{\partial \Omega}\right)=-\left.\nu \wedge(d u)\right|_{\partial \Omega} .
$$

Similarly, if $w \in C^{1}\left(\Omega, \mathcal{C}_{m}\right)$ is a function with the property that $w$ and $\delta w$ have non-tangential boundary traces at a.e. point on $\partial \Omega$ and if $N(w), N(\delta w) \in L^{p}(\partial \Omega)$, then $\left.\nu \vee w\right|_{\partial \Omega} \in L_{t a n}^{p, \delta}\left(\partial \Omega, \mathcal{C}_{m}\right)$ and

$$
\delta_{\partial}\left(\left.\nu \vee w\right|_{\partial \Omega}\right)=-\left.\nu \vee(\delta w)\right|_{\partial \Omega} .
$$

Proof of Lemma 3.1 and Lemma 3.2. The case of differential forms, i.e., when the functions involved take values in $\Lambda^{\ell} \hookrightarrow \mathcal{C}_{m}$ for some $\ell \in\{0,1, \ldots, m\}$, has been treated in MMT01 and the more general case presented here follows by gluing together such results with the help of (2.3).

Our next result, a decomposition theorem detailing the nature of the space $L_{\text {nor }}^{p, d}\left(\partial \Omega, \mathcal{C}_{3}\right)$ in the three-dimensional setting, is going to be of basic importance in our work. 
Theorem 3.3. Suppose that $\Omega$ is a bounded Lipschitz domain in $\mathbb{R}^{3}$ and assume that $1<p<\infty$. Then

$$
L_{\text {nor }}^{p, d}\left(\partial \Omega, \mathcal{C l}_{3}\right)=\nu L_{1}^{p}(\partial \Omega) \oplus * L_{\text {tan }}^{p, \text { Div }}(\partial \Omega) \oplus * L^{p}(\partial \Omega) .
$$

As a consequence, any $F \in L_{\text {nor }}^{p, d}\left(\partial \Omega, C_{3}\right)$ can be uniquely written as

$$
F=\nu f_{0}+* f_{1}+* f_{0}^{\prime},
$$

where $f_{0} \in L_{1}^{p}(\partial \Omega), f_{1} \in L_{t a n}^{p, \text { Div }}(\partial \Omega)$, and $f_{0}^{\prime} \in L^{p}(\partial \Omega)$. Furthermore, there are norm estimates naturally accompanying such a decomposition.

Proof. For the left-to-right inclusion, consider the function $h \in L_{\text {nor }}^{p, d}\left(\partial \Omega, \mathcal{C l}_{3}\right)$ and take $h=h_{0}+h_{1}+* h_{1}^{\prime}+* h_{0}^{\prime}$ to be the canonical decomposition of $h$ (compare with (2.25) and the subsequent comment). By (2.28) $h$ is normal if and only if $h_{0}=0, h_{1}^{\prime}$ is tangential and $h_{1}=f \nu$, where $f$ is a scalar-valued function in $L^{p}(\partial \Omega)$. Note that we can simply view $h_{0}^{\prime}$ as an arbitrary function in $L^{p}(\partial \Omega)$. For any test function $\varphi \in C_{0}^{\infty}\left(\mathbb{R}^{3}, \mathcal{C l}_{3}\right)$ with canonical decomposition $\varphi=\varphi_{0}+\varphi_{1}+* \varphi_{1}^{\prime}+* \varphi_{0}^{\prime}$, we know by (2.27) that

$$
\delta \varphi=-\operatorname{div} \varphi_{1}+\operatorname{curl} \varphi_{1}^{\prime}-*\left(\nabla \varphi_{0}^{\prime}\right) .
$$

In particular, choose $\varphi=* \varphi_{1}^{\prime}$. In this scenario, (3.10) and (2.25)-(2.27) allow us to write (with $1 / p+1 / p^{\prime}=1$ )

$$
\begin{aligned}
\left|\int_{\partial \Omega}\left\langle\nu, \operatorname{curl} \varphi_{1}^{\prime}\right\rangle f d \sigma\right| & =\left|\int_{\partial \Omega}\left\langle\operatorname{curl} \varphi_{1}^{\prime}, f \nu\right\rangle d \sigma\right|=\left|\int_{\partial \Omega}\left\langle\operatorname{curl} \varphi_{1}^{\prime}, h_{1}\right\rangle d \sigma\right| \\
& =\left|\int_{\partial \Omega}\left\langle\operatorname{curl} \varphi_{1}^{\prime}, h\right\rangle d \sigma\right|=\left|\int_{\partial \Omega}\langle\delta \varphi, h\rangle d \sigma\right| \\
& =\left|\int_{\partial \Omega}\left\langle\varphi, d_{\partial} h\right\rangle d \sigma\right| \leq\|\varphi\|_{L^{p^{\prime}}\left(\partial \Omega, C_{3}\right)}\left\|d_{\partial} h\right\|_{L^{p}\left(\partial \Omega, C_{3}\right)} \\
& \leq\left\|\varphi_{1}^{\prime}\right\|_{L^{p^{\prime}}\left(\partial \Omega, \mathbb{R}^{3}\right)}\left\|d_{\partial} h\right\|_{L^{p}\left(\partial \Omega, \mathcal{C}_{3}\right)} .
\end{aligned}
$$

Hence, by (3.3), $f \in L_{1}^{p}(\partial \Omega)$ plus an estimate, which shows that $h_{1}=f \nu \in \nu L_{1}^{p}(\partial \Omega)$ with norm control, as desired. To proceed, let us now specialize (3.18) to the case when $\varphi=* \varphi_{0}^{\prime}$. Then, since $h_{1}^{\prime}$ is tangential, the right-hand side of the identity (3.10) reduces to

$$
\int_{\partial \Omega}\left\langle-\nabla \varphi_{0}^{\prime}, h_{1}^{\prime}\right\rangle d \sigma=\int_{\partial \Omega}\left\langle\nabla_{\tan } \varphi_{0}^{\prime}, h_{1}^{\prime}\right\rangle d \sigma .
$$

On the other hand, the left-hand side of (3.10) can be written as

$$
\int_{\partial \Omega}\left\langle\varphi, d_{\partial} h\right\rangle d \sigma=\int_{\partial \Omega}\left\langle\varphi_{0}^{\prime}, *\left(d_{\partial} h\right)\right\rangle d \sigma
$$

The bottom line is that

$$
\int_{\partial \Omega}\left\langle\nabla_{\tan } \varphi_{0}^{\prime}, h_{1}^{\prime}\right\rangle d \sigma=\int_{\partial \Omega}\left\langle\varphi_{0}^{\prime}, *\left(d_{\partial} h\right)\right\rangle d \sigma, \quad \forall \varphi_{0}^{\prime} \in C_{0}^{\infty}\left(\mathbb{R}^{3}\right),
$$

from which we may conclude (cf. (3.5.5)) that $\operatorname{Div}\left(h_{1}^{\prime}\right)=-*\left(d_{\partial} h\right) \in L^{p}(\partial \Omega)$ plus a natural estimate. Hence $h_{1}^{\prime} \in L_{t a n}^{p \text {,Div }}(\partial \Omega)$ with norm control, as wanted. This proves the (quantitative) left-to-right inclusion in (3.16). 
Finally, in order to justify the right-to-left inclusion in (3.16), consider the function $F=\nu f_{0}+* f_{1}+* f_{0}^{\prime}$ with $f_{0} \in L_{1}^{p}(\partial \Omega), f_{1} \in L_{t a n}^{p \text {,Div }}(\partial \Omega)$ and $f_{0}^{\prime} \in L^{p}(\partial \Omega)$. Then $F \in L_{\text {nor }}^{p}\left(\partial \Omega, \mathrm{Cl}_{3}\right)$, and a calculation based on the definitions shows that

$$
d_{\partial} F=-*\left(\nu \times \nabla_{\text {tan }} f_{0}\right)+*\left(\operatorname{Div} f_{1}\right) \in L^{p}\left(\partial \Omega, \mathcal{C}_{3}\right) .
$$

Thus, $F \in L_{\text {nor }}^{p, d}\left(\partial \Omega, C_{3}\right)$ and this finishes the proof of the theorem.

The following corollary is a direct consequence of Theorem 3.3 and Hodge duality.

Corollary 3.4. Assume that $\Omega$ is a bounded Lipschitz domain in $\mathbb{R}^{3}$ and that $1<p<\infty$. Then

$$
L_{\text {tan }}^{p, \delta}\left(\partial \Omega, \ell_{3}\right)=L^{p}(\partial \Omega) \oplus L_{t a n}^{p, \text { Div }}(\partial \Omega) \oplus *\left(\nu L_{1}^{p}(\partial \Omega)\right) .
$$

As a consequence, any function $G \in L_{t a n}^{p, \delta}\left(\partial \Omega, C_{3}\right)$ will be uniquely written as

$$
G=g_{0}+g_{1}+*\left(\nu g_{0}^{\prime}\right),
$$

where $g_{0} \in L^{p}(\partial \Omega), g_{1} \in L_{\text {tan }}^{p \text {, Div }}(\partial \Omega)$, and $g_{0}^{\prime} \in L_{1}^{p}(\partial \Omega)$. In addition, there are natural estimates accompanying such a decomposition.

We conclude this section by presenting a structural result pertaining to the nature of the space of $L^{p}$ normal $\mathrm{Cl}_{4}$-valued functions having their boundary exterior derivatives in $L^{p}$.

Theorem 3.5. Suppose that $\Omega$ is a bounded Lipschitz domain in $\mathbb{R}^{3}$ and assume that $1<p<\infty$. Then the following (algebraic and topological) decomposition holds:

$$
L_{\text {nor }}^{p, d}\left(\partial \Omega, \mathcal{C l}_{4}\right)=L_{\text {nor }}^{p, d}\left(\partial \Omega, \mathcal{C l}_{3}\right) \oplus\left(e_{4} \odot L_{\text {nor }}^{p, d}\left(\partial \Omega, \mathcal{C l}_{3}\right)\right) .
$$

Thus, any function $f \in L_{\text {nor }}^{p, d}\left(\partial \Omega, \mathcal{C l}_{4}\right)$ can be uniquely written as

$$
f=F+e_{4} \odot \widetilde{F}, \quad \text { for some } F, \widetilde{F} \in L_{\text {nor }}^{p, d}\left(\partial \Omega, \mathcal{C l}_{3}\right),
$$

and a naturally accompanying estimate holds. Moreover, the decomposition (3.27) can be further refined by writing

$$
f=\nu f_{0}+* f_{1}+* f_{0}^{\prime}+e_{4} \odot\left(\nu \widetilde{f}_{0}+* \widetilde{f}_{1}+* \widetilde{f}_{0}^{\prime}\right)
$$

uniquely and quantitatively, for some $f_{0}, \widetilde{f}_{0} \in L_{1}^{p}(\partial \Omega), f_{1}, \widetilde{f}_{1} \in L_{\text {tan }}^{p, \text { Div }}(\partial \Omega)$, and $f_{0}^{\prime}, \widetilde{f}_{0}^{\prime} \in L^{p}(\partial \Omega)$.

Proof. First, (3.26) is a direct consequence of (3.10)-(3.11) and Lemma 2.5 and, in turn, (3.26) yields (3.27). With this in hand, (3.28) follows with the help of Theorem 3.3 .

\section{Integral operators of CaUchy type}

This section is devoted to introducing and studying integral operators of CliffordCauchy type associated with the perturbed Dirac operator $\mathbb{D}_{k}$ in Lipschitz domains, along the lines of the general procedure described in MM03. Their integral kernels are based on the fundamental solution for $\mathbb{D}_{k}$ which, in turn, is constructed starting from the fundamental solution of the Helmholtz operator, which we now review. 
Let $E_{m}(z ; x)$ be the fundamental solution of the Helmholtz operator $-\Delta-z$ in $\mathbb{R}^{m}$, $m \in \mathbb{N}, m \geq 2$, namely

$$
E_{m}(z ; x):= \begin{cases}\frac{i}{4}\left(\frac{2 \pi|x|}{z^{1 / 2}}\right)^{(2-m) / 2} H_{(m-2) / 2}^{(1)}\left(z^{1 / 2}|x|\right), & m \geq 2, z \in \mathbb{C} \backslash\{0\}, \\ \frac{-1}{2 \pi} \ln (|x|), & m=2, z=0, \\ \frac{1}{(m-2) \omega_{m-1}}|x|^{2-m}, & m \geq 3, z=0, \\ \operatorname{Im}\left(z^{1 / 2}\right) \geq 0, & x \in \mathbb{R}^{m} \backslash\{0\},\end{cases}
$$

where $H_{\mu}^{(1)}(\cdot)$ denotes the Hankel function of the first kind with index $\mu \geq 0$ (cf. [AS72, Sect. 9.1]) and $\omega_{m-1}=2 \pi^{m / 2} / \Gamma(m / 2)$ (with $\Gamma$ denoting the Gamma function; cf. [AS72, Sect. 6.1]) represents the area of the unit sphere $S^{m-1}$ in $\mathbb{R}^{m}$. Since $-\Delta-z$ depends analytically on $z$, it follows that $E_{m}(z ; x)$ depends analytically on $z$. Also, as $z \rightarrow 0, E_{m}(z, x), x \in \mathbb{R}^{m} \backslash\{0\}$ is continuous for $m \geq 3$, i.e.,

$$
E_{m}(z ; x) \underset{z \rightarrow 0}{=} E_{m}(0, x)=\frac{1}{(m-2) \omega_{m-1}}|x|^{2-m}, \quad x \in \mathbb{R}^{m} \backslash\{0\}, \quad m \geq 3,
$$

but is discontinuous for $m=2$ since whenever $x \in \mathbb{R}^{2} \backslash\{0\}$,

$$
E_{2}(z ; x) \underset{z \rightarrow 0}{=} \frac{-1}{2 \pi} \ln \left(z^{1 / 2}|x| / 2\right)\left[1+O\left(z|x|^{2}\right)\right]+\frac{1}{2 \pi} \psi(1)+O\left(z|x|^{2}\right) .
$$

Here $\psi:=\Gamma^{\prime} / \Gamma$ denotes the digamma function (cf. AS72, Sect.6.3]). Thus, we simply define $E_{2}(0 ; x):=\frac{-1}{2 \pi} \ln (|x|), x \in \mathbb{R}^{2} \backslash\{0\}$, as in (4.1). To estimate $E_{m}$, recall that (cf. [AS72, Sect. 9.1])

$$
H_{(m-2) / 2}^{(1)}(\cdot)=J_{(m-2) / 2}(\cdot)+i Y_{(m-2) / 2}(\cdot),
$$

with $J_{\mu}$ and $Y_{\mu}$ the regular and irregular Bessel functions, respectively. Based on the asymptotic expansions of these functions, the following result summarizing the behavior of $E_{m}(z ; x)$ and its derivatives up to the second order as $|x| \rightarrow 0$ has been proved in GeMi08. This is important for proving that the difference between two layer potentials based on fundamental solutions for two different Helmholtz operators is compact on various function spaces.

Lemma 4.1. Fix $z \in \mathbb{C} \backslash\{0\}$. Then the fundamental solution $E_{m}(z ; \cdot)$ of the Helmholtz operator $-\Delta-z$ and its derivatives up to the second order satisfy the following estimates for $0<|x|<R$, with $R>0$ fixed:

$$
\begin{array}{r}
\left|E_{m}(z ; x)-E_{m}(0 ; x)\right| \leq \begin{cases}C, & m=2,3, \\
C[|\ln (|x|)|+1], & m=4, \\
C\left[|x|^{4-m}+1\right], & m \geq 5,\end{cases} \\
\left|\partial_{j} E_{m}(z ; x)-\partial_{j} E_{m}(0 ; x)\right| \leq \begin{cases}C, & m=2,3, \\
C\left[|x|^{3-m}+1\right], & m \geq 4,\end{cases} \\
\left|\partial_{j} \partial_{k} E_{m}(z ; x)-\partial_{j} \partial_{k} E_{m}(0 ; x)\right| \leq \begin{cases}C[|\ln (|x|)|+1], & m=2, \\
C\left[|x|^{2-m}+1\right], & m \geq 3 .\end{cases}
\end{array}
$$

Above, $C=C(R, m, z)$ is a constant and $\partial_{j}=\partial / \partial x_{j}$ for $1 \leq j \leq m$. 
Throughout, given $m \in \mathbb{N}, m \geq 2$, fixed, for each $k \in \mathbb{C}$ we agree to abbreviate (dropping the dependence on $m$, whose value should be clear from the context)

$$
\Phi_{k}(x):=-E_{m}\left(k^{2} ; x\right), \quad x \in \mathbb{R}^{m} \backslash\{0\} .
$$

As such, $\Phi_{k}$ is a radial fundamental solution for the Helmholtz operator $\Delta+k^{2}$ in $\mathbb{R}^{m}$, and has the property that, when $m=3$ and $k \in \mathbb{C}$ is such that $\operatorname{Im} k \geq 0$,

$$
\Phi_{k}(x)=-\frac{e^{i k|x|}}{4 \pi|x|}, \quad x \in \mathbb{R}^{3} \backslash\{0\} .
$$

Let $\Omega$ be a bounded Lipschitz domain in $\mathbb{R}^{m}$ and fix some complex number $k$. Recall that the single-layer acoustic potential operator associated to $\Omega$ is defined for a function $f: \partial \Omega \rightarrow \mathcal{C}_{m}$ by

$$
\left(\mathscr{S}_{k} f\right)(x):=\int_{\partial \Omega} \Phi_{k}(x-y) f(y) d \sigma_{y}, \quad x \in \mathbb{R}^{m} \backslash \partial \Omega .
$$

By design, the single-layer acoustic potential is a null-solution of the Helmholtz operator $\Delta+k^{2}$ in $\mathbb{R}^{m} \backslash \partial \Omega$. Let us also define its boundary version by setting, for $f: \partial \Omega \rightarrow \mathcal{C l}_{m}$,

$$
\left(S_{k} f\right)(x):=\int_{\partial \Omega} \Phi_{k}(x-y) f(y) d \sigma_{y}, \quad x \in \partial \Omega .
$$

We shall now define the operator $\mathscr{C}_{k}$, mapping Clifford algebra-valued functions defined on the boundary into functions defined in the complement of the boundary as

$$
\mathscr{C}_{k}:=\mathbb{D}_{k} \mathscr{S}_{k}=d \mathscr{S}_{k}+\delta \mathscr{S}_{k}+k e_{4} \odot \mathscr{S}_{k},
$$

or, more explicitly, for each $f: \partial \Omega \rightarrow \mathcal{C}_{m}$,

$$
\left(\mathscr{C}_{k} f\right)(x)=\int_{\partial \Omega}\left(\mathbb{D}_{k} \Phi_{k}\right)(x-y) \odot f(y) d \sigma_{y}, \quad x \in \mathbb{R}^{m} \backslash \partial \Omega .
$$

The reader is alerted to the fact that while (4.13) is closely related to what one might consider to be the standard definition of the Cauchy operator in the Clifford algebra context, cf. e.g., BDS82, GM91, Mi94, $\mathscr{C}_{k}$ differs from this standard definition up to a composition with the operator of Clifford multiplication by the unit normal. We will nonetheless continue to refer to (4.13) simply as a Cauchy operator. Thanks to (4.12) and the second formula in (2.15), this Cauchy operator is annihilated by the perturbed Dirac operator $\mathbb{D}_{k}$. Its principle value boundary version is given by

$$
\begin{aligned}
\left(C_{k} f\right)(x) & :=\text { p.v. } \int_{\partial \Omega}\left(\mathbb{D}_{k} \Phi_{k}\right)(x-y) \odot f(y) d \sigma_{y} \\
& :=\lim _{\varepsilon \rightarrow 0^{+}} \int_{\substack{|x-y|>\varepsilon \\
y \in \partial \Omega}}\left(\mathbb{D}_{k} \Phi_{k}\right)(x-y) \odot f(y) d \sigma_{y} \quad x \in \partial \Omega,
\end{aligned}
$$

again, for each $f: \partial \Omega \rightarrow \mathcal{C}_{m}$. We also find it useful to work with the double-layer acoustic potential operator, acting on $f: \partial \Omega \rightarrow \mathcal{C}_{m}$ according to (with $\partial_{\nu}$ denoting the normal derivative)

$$
K_{k} f(x):=\text { p.v. } \int_{\partial \Omega} \partial_{\nu_{y}}\left[\Phi_{k}(x-y)\right] f(y) d \sigma_{y}, \quad x \in \partial \Omega,
$$


and we denote its formal transpose by $K_{k}^{\top}$. Here $\partial_{\nu_{y}}$ denotes the normal derivative in the variable $y$. It can then be readily verified, solely based on definitions, that the following identities hold for a scalar-valued function $f$ defined on $\partial \Omega$ :

$$
K_{k} f=-\operatorname{div} S_{k}(\nu f) \text { and } K_{k}^{\top} f=\nu \cdot \nabla S_{k} f .
$$

When $m=3$, the principle value magnetic dipole operator is defined as

$$
M_{k} f(x):=\nu(x) \times\left(\text { p.v. } \int_{\partial \Omega} \operatorname{curl}_{x}\left\{\Phi_{k}(x-y) f(y)\right\} d \sigma_{y}\right), \quad x \in \partial \Omega,
$$

whenever $f$ is a vector field defined on $\partial \Omega$.

Finally, we introduce two more pieces of notation. Specifically, set

$$
d S_{k} h(x):=\text { p.v. } \int_{\partial \Omega}\left(\nabla \Phi_{k}\right)(x-y) \wedge h(y) d \sigma_{y}, \quad x \in \partial \Omega,
$$

and

$$
\delta S_{k} h(x):=- \text { p.v. } \int_{\partial \Omega}\left(\nabla \Phi_{k}\right)(x-y) \vee h(y) d \sigma_{y}, \quad x \in \partial \Omega,
$$

where $h$ is a Clifford algebra-valued function defined on $\partial \Omega$.

Before going any further, some comments are in order. First, we wish to stress that $d S_{k}$ does not signify that the exterior derivative operator $d$ has been applied to the boundary version of the acoustic single layer $S_{k}$ but, rather, that this is a convenient symbol to denote the principal-value operator on the right-hand side of (4.18). A similar comment applies to $\delta S_{k}$. Second, the specific nature of the various principal-value singular integral operators introduced so far is such that they fall under the scope of the (classical, by now) Calderón-Zygmund theory on Lipschitz surfaces (cf. CMM82 for more details on this matter). In particular, all the aforementioned operators are meaningfully defined a.e. on the boundary whenever applied to functions with $L^{p}$ components, $p \in(1, \infty)$. Furthermore, appropriate $L^{p}$ estimates, again when $p \in(1, \infty)$, hold in each case.

Moving on, we present some important intertwining properties of the operators $d, \delta$ and their boundary versions, i.e., $d_{\partial}, \delta_{\partial}$, on the one hand, and the acoustic single layer potential operator on the other hand.

Lemma 4.2. Let $\Omega$ be a bounded Lipschitz domain in $\mathbb{R}^{m}, 1<p<\infty$ and $k \in \mathbb{C}$. Then

$$
\begin{aligned}
& d \mathscr{S}_{k} F=\mathscr{S}_{k}\left(d_{\partial} F\right) \quad \text { for each } F \in L_{\text {nor }}^{p, d}\left(\partial \Omega, \mathcal{C}_{m}\right) \text {, } \\
& \delta \mathscr{S}_{k} G=\mathscr{S}_{k}\left(\delta_{\partial} G\right) \quad \text { for each } G \in L_{t a n}^{p, \delta}\left(\partial \Omega, \mathcal{C l}_{m}\right) \text {. }
\end{aligned}
$$

Moreover, the boundary versions of (4.20)-(4.21) are also valid, namely

$$
\begin{aligned}
& d S_{k} F=S_{k}\left(d_{\partial} F\right) \quad \text { for each } F \in L_{\text {nor }}^{p, d}\left(\partial \Omega, \mathcal{C l}_{m}\right), \\
& \delta S_{k} G=S_{k}\left(\delta_{\partial} G\right) \quad \text { for each } \quad G \in L_{\text {tan }}^{p, \delta}\left(\partial \Omega, \mathcal{C l}_{m}\right) .
\end{aligned}
$$

Proof. This can be adapted from similar results proved for differential forms in MMT01, much as explained in the proof of Lemmas 3.13.2

Given that our main well-posedness results for the transmission problems we study in this paper are formulated in the three-dimensional setting, from now on we will restrict our attention to the case when $m=3$. First we shall deal with nontangential maximal function estimates for the Cauchy operator, a result of basic 
importance for subsequent work. Prior to stating this result, the reader is advised to recall the definition (1.5)).

Theorem 4.3. Let $\Omega \subset \mathbb{R}^{3}$ be an arbitrary bounded Lipschitz domain. Then for each $k \in \mathbb{C}$ and $p \in(1, \infty)$ there exists a finite constant $C=C(k, \partial \Omega, p)>0$ such that if $f \in L_{\text {nor }}^{p, d}\left(\partial \Omega, \mathcal{C l}_{4}\right)$ and $u:=\mathscr{C}_{k} f$ in $\Omega$, one has

$$
\|N(u)\|_{L^{p}(\partial \Omega)}+\|N(d u)\|_{L^{p}(\partial \Omega)}+\|N(\delta u)\|_{L^{p}(\partial \Omega)} \leq C\|f\|_{L_{n o r}^{p, d}\left(\partial \Omega, C_{4}\right)} .
$$

Proof. By (4.12), we have

$$
u=d \mathscr{S}_{k} f+\delta \mathscr{S}_{k} f+k e_{4} \odot \mathscr{S}_{k} f \quad \text { in } \Omega .
$$

From the results proved in CMM82 and, by now, well-understood techniques in the theory of Calderón-Zygmund singular integral operators, it follows that

$$
\|N(u)\|_{L^{p}(\partial \Omega)} \leq C\|f\|_{L^{p}\left(\partial \Omega, C_{4}\right)} \leq C\|f\|_{L_{\text {nor }}^{p, d}\left(\partial \Omega, \mathcal{C}_{4}\right)} .
$$

Next, by (2.17) and Lemma 2.5] we obtain

$$
\begin{aligned}
d u & =d^{2} \mathscr{S}_{k} f+d \delta \mathscr{S}_{k} f+k d\left(e_{4} \odot \mathscr{S}_{k} f\right) \\
& =d \delta \mathscr{S}_{k} f-k e_{4} \odot d \mathscr{S}_{k} f \quad \text { in } \Omega .
\end{aligned}
$$

Using the identity $d \delta=-\delta d-\left(\Delta+k^{2}\right)+k^{2}$ and the fact, as already pointed out, the Helmholtz operator annihilates the acoustic single-layer $\mathscr{S}_{k}$, we arrive at

$$
d u=-\delta d \mathscr{S}_{k} f+k^{2} \mathscr{S}_{k} f-k e_{4} \odot d \mathscr{S}_{k} f \quad \text { in } \Omega .
$$

To proceed, recall from Lemma 4.2 that $d \mathcal{S}_{k} f=\mathscr{S}_{k}\left(d_{\partial} f\right)$. Thus,

$$
d u=-\delta \mathscr{S}_{k}\left(d_{\partial} f\right)+k^{2} \mathscr{S}_{k} f-k e_{4} \odot d \mathscr{S}_{k} f \quad \text { in } \Omega .
$$

At this stage, the Calderón-Zygmund theory applies and, since $d_{\partial} f$ has $L^{p}$ components (given that $f \in L_{\text {nor }}^{p, d}\left(\partial \Omega, C_{4}\right)$ ), yields

$$
\|N(d u)\|_{L^{p}(\partial \Omega)} \leq C\|f\|_{L_{\text {nor }}^{p, d}\left(\partial \Omega, C_{4}\right)} .
$$

The case of $N(\delta u)$ is treated in a similar fashion. Concretely, we write

$$
\delta u=\delta \mathscr{S}_{k}\left(d_{\partial} f\right)-k e_{4} \odot \delta \mathscr{S}_{k} f, \quad \text { in } \Omega,
$$

at which point the same analysis as before applies.

We next discuss jump formulas for derivatives of the acoustic single layer potential operator $\mathscr{S}_{k}$. As a preamble, the reader is advised to review (1.1) and (1.6).

Lemma 4.4. Assume that $\Omega \subset \mathbb{R}^{3}$ is an arbitrary Lipschitz domain and fix $k \in \mathbb{C}$ and $p \in(1, \infty)$, arbitrary. Then for any function $h \in L^{p}\left(\partial \Omega, \mathcal{C}_{4}\right)$ the following jump-formulas are valid:

$$
\begin{aligned}
& \left.d \mathscr{S}_{k} h\right|_{\partial \Omega \pm}(x)=\mp \frac{1}{2}(\nu \wedge h)(x)+d S_{k} h(x), \\
& \left.\delta \mathscr{S}_{k} h\right|_{\partial \Omega \pm}(x)= \pm \frac{1}{2}(\nu \vee h)(x)+\delta S_{k} h(x),
\end{aligned}
$$

at a.e. point $x \in \partial \Omega$.

Proof. When $h$ is a differential form, these formulas have been proved in MMT01, and the extension to the more general case presented here is done by gluing together such results with the help of the decomposition presented in (2.3). 
The stage is now set for proving jump-formulas for the Clifford-Cauchy operator $\mathscr{C}_{k}$. General results of this type have been established in [MMT01, Theorem 1.1, p. 7 and Theorem 2.9, p. 21].

Theorem 4.5. Let $\Omega \subset \mathbb{R}^{3}$ be an arbitrary bounded Lipschitz domain and suppose that $k \in \mathbb{C}$ and that $p \in(1, \infty)$. Then for any $h \in L^{p}\left(\partial \Omega, \mathcal{C}_{4}\right)$ one has

$$
\left.\left(\mathscr{C}_{k} h\right)\right|_{\partial \Omega \pm}=\left(\mp \frac{1}{2} \nu \odot I+C_{k}\right) h,
$$

where I denotes the identity operator. As a consequence,

$$
\begin{aligned}
& \left.\nu \wedge\left(\mathscr{C}_{k} h\right)\right|_{\partial \Omega \pm}= \pm \frac{1}{2} h_{\text {nor }}+\nu \wedge C_{k} h, \\
& \left.\nu \vee\left(\mathscr{C}_{k} h\right)\right|_{\partial \Omega \pm}=\mp \frac{1}{2} h_{\text {tan }}+\nu \vee C_{k} h .
\end{aligned}
$$

Proof. Based on the very definition of $\mathscr{C}_{k}$ we may write

$$
\left.\mathscr{C}_{k} h\right|_{\partial \Omega \pm}=\left.d \mathscr{S}_{k} h\right|_{\partial \Omega \pm}+\left.\delta \mathscr{S}_{k} h\right|_{\partial \Omega \pm}+\left.k e_{4} \odot \mathscr{S}_{k} h\right|_{\partial \Omega \pm} .
$$

Now, thanks to (4.32), (4.33) and since, due to the weak singularity of its integral kernel, the acoustic single layer-operator satisfies

$$
\left.\mathscr{S}_{k} h\right|_{\partial \Omega \pm}=S_{k} h
$$

we obtain

$$
\begin{aligned}
\left.\mathscr{C}_{k} h\right|_{\partial \Omega \pm} & =\mp \frac{1}{2}(\nu \wedge h)+d S_{k} h+\left( \pm \frac{1}{2}\right)(\nu \vee h)+\delta S_{k} h+k e_{4} \odot S_{k} h \\
& =\mp \frac{1}{2}(\nu \wedge h-\nu \vee h)+C_{k} h=\mp \frac{1}{2} \nu \odot h+C_{k} h .
\end{aligned}
$$

This establishes (4.34). Finally, (4.35)-(4.36) are consequences of (4.34) and elements of Clifford calculus reviewed earlier. This finishes the proof of the theorem.

We conclude this section with a discussion of the decay and reproducing properties of the Clifford-Cauchy integral operator. Given a bounded Lipschitz domain $\Omega \subseteq \mathbb{R}^{3}, k \in \mathbb{C}$, and $p \in(1, \infty)$, the following equivalence holds (cf. Mi94 for results of this type):

$$
\begin{aligned}
& u \in C^{\infty}\left(\Omega, \mathcal{C}_{4}\right) \text { is } k \text {-monogenic, and } N u \in L^{p}(\partial \Omega) \text { if and only if } \\
& \left.u\right|_{\partial \Omega} \text { exists, belongs to } L^{p}\left(\partial \Omega, \mathcal{C l}_{4}\right) \text {, and } u=\mathscr{C}_{k}\left(\left.\nu \odot u\right|_{\partial \Omega}\right) \text { in } \Omega \text {. }
\end{aligned}
$$

Dealing with similar issues in the unbounded Lipschitz domain $\Omega_{-}:=\mathbb{R}^{3} \backslash \bar{\Omega}$ requires imposing a suitable decay condition at infinity. The specific nature of such a decay condition in the class of $k$-monogenic functions $u: \Omega_{-} \rightarrow \mathrm{Cl}_{4}$ depends on the nature of the wave number $k$. When $\operatorname{Im} k>0$, then any $k$-monogenic function $u: \Omega_{-} \rightarrow C_{4}$ which, say, is bounded at infinity actually decays exponentially at infinity. The case when $k \in \mathbb{R} \backslash\{0\}$ is more subtle, and the appropriate decay condition in this setting has been identified in [McM99] as being

$$
\lim _{|x| \rightarrow \infty}\left(|x|-i e_{4} \odot x\right) \odot u(x)=0 .
$$

The following result, which is the counterpart of (4.40) for the unbounded domain $\Omega_{-}$, has been proved in [McM99. 
Proposition 4.6. Assume that $\Omega \subseteq \mathbb{R}^{3}$ is a bounded Lipschitz domain, that $k \in$ $\mathbb{C} \backslash\{0\}$ has $\operatorname{Im} k \geq 0$ and that $p \in(1, \infty)$. As in the past, set $\Omega_{-}:=\mathbb{R}^{3} \backslash \bar{\Omega}$. Then the following equivalence is valid:

$$
\begin{aligned}
& u \in C^{\infty}\left(\Omega_{-}, C_{4}\right) \text { is k-monogenic, } N u \in L^{p}(\partial \Omega) \text { and u satisfies (4.41) } \\
& \text { if and only if } \\
& \left.u\right|_{\partial \Omega} \text { exists, belongs to } L^{p}\left(\partial \Omega, C_{4}\right), \text { and } u=\mathscr{C}_{k}\left(\left.\nu \odot u\right|_{\partial \Omega}\right) \text { in } \Omega_{-} .
\end{aligned}
$$

It is worth pointing out that, in the Clifford algebra $C_{4}$, the quantity $|x|-i e_{4} \odot x$ (appearing in (4.41)) is a zero divisor so, at least at first glance, it appears that the information encoded in (4.41) is that certain combinations of components vanish at infinity. Nonetheless, we have the following result.

Theorem 4.7. Assume that $u$ is a $\mathrm{Cl}_{4}$-valued function which is $k$-monogenic, for some $k \in \mathbb{R} \backslash\{0\}$, in the neighborhood of infinity in $\mathbb{R}^{3}$. Then $u$ satisfies (4.41) if and only if each of its scalar components satisfies Sommerfeld's radiation condition (cf. (1.8)).

The proof of Theorem 4.7 requires a number of preliminaries, which we address first. The following proposition, proved in [DMM06], describes an extension of the unit normal to a smooth hypersurface enjoying a number of useful properties.

Proposition 4.8. For a $C^{2}$ surface $\mathcal{S} \subseteq \mathbb{R}^{n}$ there exist a neighborhood $\mathcal{U}$ of $\mathcal{S}$ along with a vector field $\nu=\left(\nu_{1}, \ldots, \nu_{n}\right) \in C^{1}(\mathcal{U})$ with the following properties:

(i) $|\nu|=1$ in $\mathcal{U}$;

(ii) $\left.\nu\right|_{\mathcal{S}}$ coincides with the unit normal to $\mathcal{S}$;

(iii) $\partial_{\nu} \nu=0$ on $\mathcal{S}$, i.e., $\partial_{\nu} \nu_{j}=0$ on $\mathcal{S}$ for all $j \in\{1,2, \ldots, n\}$;

(iv) $d \nu=0$ on $\mathcal{S}$, i.e., $\partial_{i} \nu_{j}=\partial_{j} \nu_{i}$ on $\mathcal{S}$ for all $i, j \in\{1,2, \ldots, n\}$.

(v) $\left.(\operatorname{div} \nu)\right|_{\mathcal{S}}=(n-1) \mathcal{H}$, where $\mathcal{H}$ stands for the mean curvature of $\mathcal{S}$.

If $\mathcal{S} \subseteq \mathbb{R}^{n}$ is a $C^{2}$ surface and $\nu$ is an extension of the unit normal to $\mathcal{S}$ in a neighborhood $\mathcal{U}$ of $\mathcal{S}$ as in Proposition 4.8, then for each $j, \ell \in\{1,2, \ldots, n\}$ we define the first-order partial differential operator $\partial_{\tau_{j \ell}}:=\nu_{j} \partial_{\ell}-\nu_{\ell} \partial_{j}$ in $\mathcal{U}$. Then for each $j \in\{1, \ldots, n\}$, using the summation convention over repeated indices, in the open set $\mathcal{U}$ we may write

$$
\partial_{j}=\nu_{\ell} \nu_{\ell} \partial_{j}=\nu_{\ell}\left(\nu_{\ell} \partial_{j}-\nu_{j} \partial_{\ell}\right)+\nu_{\ell} \nu_{j} \partial_{\ell}=\nu_{\ell} \partial_{\tau_{\ell j}}+\nu_{j} \partial_{\nu}
$$

In turn, using the product rule and (4.43) , for each $i, j \in\{1, \ldots, n\}$ we also have on $\mathcal{U}$

$$
\partial_{\tau_{\ell j}}\left(\nu_{i} \nu_{\ell}\right)=\nu_{\ell} \partial_{\tau_{\ell j}} \nu_{i}+\nu_{i} \partial_{\tau_{\ell j}} \nu_{\ell}=\left(\partial_{j}-\nu_{j} \partial_{\nu}\right) \nu_{i}+\nu_{i}\left(\nu_{\ell} \partial_{j} \nu_{\ell}-\nu_{j} \partial_{\ell} \nu_{\ell}\right)
$$

Hence, from (4.44) and (iii), (i), (v) in Proposition 4.8 it follows that

$$
\left.\left[\partial_{\tau_{\ell j}}\left(\nu_{i} \nu_{\ell}\right)\right]\right|_{\mathcal{S}}=\left.\left(\partial_{j} \nu_{i}\right)\right|_{\mathcal{S}}-(n-1) \nu_{i} \nu_{j}(n-1) \mathcal{H}
$$

Finally, since (iv) in Proposition 4.8 implies that the right-hand side of (4.45) is symmetric in $i, j$, we may conclude that

$$
\left.\left[\partial_{\tau_{\ell j}}\left(\nu_{i} \nu_{\ell}\right)\right]\right|_{\mathcal{S}}=\left.\left[\partial_{\tau_{\ell i}}\left(\nu_{j} \nu_{\ell}\right)\right]\right|_{\mathcal{S}} \quad \text { for every } i, j \in\{1, \ldots, n\}
$$

This is going to be of importance shortly. For now, we wish to record an integration by parts formula on a surface involving the operators $\partial_{\tau_{\ell j}}$. 
Lemma 4.9. Let $\mathcal{S} \subseteq \mathbb{R}^{n}$ be a compact $C^{2}$ surface and assume that $\nu$ is the extension of the unit normal to $\mathcal{S}$ in a neighborhood $\mathcal{U}$ of $\mathcal{S}$ described in Proposition 4.8. Also, denote by $\sigma$ the surface measure on $\mathcal{S}$ and fix $j, \ell \in\{1,2, \ldots, n\}$. Then for every function $u, v \in C^{1}(\mathcal{U})$ one has

$$
\int_{\mathcal{S}} u\left(\partial_{\tau_{j \ell}} v\right) d \sigma=\int_{\mathcal{S}}\left(\partial_{\tau_{\ell j}} u\right) v d \sigma
$$

Proof. Assuming first that $u, v \in C_{c}^{2}(\mathcal{U})$, we use successive integration by parts in order to write

$$
\begin{aligned}
\int_{\mathcal{S}} u\left(\partial_{\tau_{j \ell}} v\right) d \sigma & =\int_{\mathcal{S}} u\left(\nu_{\ell} \partial_{j} v-\nu_{j} \partial_{\ell} v\right) d \sigma=\int_{\mathcal{U}}\left(\partial_{j}\left(u \partial_{\ell} v\right)-\partial_{\ell}\left(u \partial_{j} v\right)\right) d x \\
& =\int_{\mathcal{U}}\left(\partial_{\ell}\left(v \partial_{j} u\right)-\partial_{j}\left(v \partial_{\ell} u\right)\right) d x=\int_{\mathcal{S}}\left(\partial_{\tau_{\ell j}} u\right) v d \sigma
\end{aligned}
$$

Finally, the more general case when $u, v \in C^{1}(\mathcal{U})$ is easily reduced to the previous situation by truncating $u, v$ with a smooth, compactly supported function which is identically one near $\mathcal{S}$, and then using a standard mollifying and limiting argument.

We are now well prepared to deal with the

Proof of Theorem 4.7. Let $k \in \mathbb{R} \backslash\{0\}$ and let $u: \mathbb{R}^{3} \backslash \overline{B(0, R)} \rightarrow \mathcal{C}_{4}$, where $R \in(0, \infty)$, be a $k$-monogenic function whose scalar components satisfy Sommerfeld's radiation condition. Fix a bounded domain $\Omega \subseteq \mathbb{R}^{3}$ of class $C^{2}$ with the property that $\overline{B(0, R)} \subseteq \Omega$ and denote by $\nu$ and $\sigma$ the outward unit normal and surface measure to $\partial \Omega$. Finally, extend $\nu$ to an open neighborhood $\mathcal{U}$ of $\partial \Omega$, which is contained in $\mathbb{R}^{3} \backslash \overline{B(0, R)}$, as in Proposition 4.8. Since the individual scalar components of $u$ are also annihilated by the Helmholtz operator $\Delta+k^{2}$ (cf. (1.12)), it follows from [CK92, Theorem 2.4, p. 18] that

$$
\begin{gathered}
u(x)=\int_{\partial \Omega} \partial_{\nu_{y}}\left[\Phi_{k}(x-y)\right] u(y) d \sigma_{y}-\int_{\partial \Omega} \Phi_{k}(x-y)\left(\partial_{\nu} u\right)(y) d \sigma_{y}, \\
\text { whenever } \quad x \in \mathbb{R}^{3} \backslash \bar{\Omega},
\end{gathered}
$$

where the normal derivative $\partial_{\nu}$ is applied to $u$ componentwise. On the other hand, based on (4.43) and the monogenicity of $u$, we may write in $\mathcal{U}$ (using the summation convention over repeated indices in the set $\{1,2,3\}$ )

$$
\begin{gathered}
0=\mathbb{D}_{k} u=e_{j} \odot \partial_{j} u+k e_{4} \odot u=\nu_{\ell} e_{j} \odot \partial_{\tau_{\ell j}} u+\nu_{j} e_{j} \odot \partial_{\nu} u+k e_{4} \odot u \\
=\nu_{\ell} e_{j} \odot \partial_{\tau_{\ell j}} u+\nu \odot \partial_{\nu} u+k e_{4} \odot u .
\end{gathered}
$$

In turn, using the fact that $\nu^{2}=-1$, this allows us to solve for $\partial_{\nu} u$. We thus obtain

$$
\partial_{\nu} u=\nu_{\ell} \nu \odot e_{j} \odot \partial_{\tau_{\ell j}} u-k e_{4} \odot \nu \odot u \quad \text { in } \mathcal{U} \text {. }
$$

By replacing this in the last integral in (4.49), we therefore obtain for each fixed $x \in \mathbb{R}^{3} \backslash \bar{\Omega}$ that

$$
\begin{aligned}
\int_{\partial \Omega} \Phi_{k}(x-y)\left(\partial_{\nu} u\right)(y) d \sigma_{y}= & \int_{\partial \Omega} \Phi_{k}(x-y) \nu_{\ell}(y) \nu(y) \odot e_{j} \odot\left(\partial_{\tau_{\ell j}} u\right)(y) d \sigma_{y} \\
& -k \int_{\partial \Omega} \Phi_{k}(x-y) e_{4} \odot \nu(y) \odot u(y) d \sigma_{y} .
\end{aligned}
$$


Let us further consider the first integral on the right-hand side of (4.52). Based on Lemma 4.9. we may integrate by parts on $\partial \Omega$ and transform this as follows:

$$
\begin{aligned}
\int_{\partial \Omega} & \Phi_{k}(x-y) \nu_{\ell}(y) \nu(y) \odot e_{j} \odot\left(\partial_{\tau_{\ell j}} u\right)(y) d \sigma_{y} \\
& =\int_{\partial \Omega} \Phi_{k}(x-y) \nu_{\ell}(y) \nu_{i}(y) e_{i} \odot e_{j} \odot\left(\partial_{\tau_{\ell j}} u\right)(y) d \sigma_{y} \\
& =\int_{\partial \Omega} \partial_{\tau_{j \ell}(y)}\left[\Phi_{k}(x-y) \nu_{\ell}(y) \nu_{i}(y)\right] e_{i} \odot e_{j} \odot u(y) d \sigma_{y} \\
& =\int_{\partial \Omega} \partial_{\tau_{j \ell}(y)}\left[\Phi_{k}(x-y)\right] \nu_{\ell}(y) \nu_{i}(y) e_{i} \odot e_{j} \odot u(y) d \sigma_{y} .
\end{aligned}
$$

In the last step above we have used the product rule for $\partial_{\tau_{j \ell}}$ together with the following cancellation property:

$$
\begin{aligned}
\partial_{\tau_{j \ell}}\left(\nu_{\ell} \nu_{i}\right) e_{i} \odot e_{j} & =-\sum_{\ell} \sum_{i} \partial_{\tau_{i \ell}}\left(\nu_{\ell} \nu_{i}\right)+\sum_{\ell} \sum_{i \neq j} \partial_{\tau_{j \ell}}\left(\nu_{\ell} \nu_{i}\right) e_{i} \odot e_{j} \\
& =0+0=0
\end{aligned}
$$

given that $\partial_{\tau_{i \ell}}$ is anti-symmetric in $i, \ell$ while $\nu_{\ell} \nu_{i}$ is symmetric in $i, \ell$, and given that the expression $\partial_{\tau_{j \ell}}\left(\nu_{\ell} \nu_{i}\right)$ is symmetric in $i, j$ (by (4.46)) while $e_{i} \odot e_{j}$ is antisymmetric in $i, j$ for $i \neq j$. Having clarified this issue, we now proceed to reorganize the last integral in (4.53) as follows:

$$
\begin{aligned}
\int_{\partial \Omega} \partial_{\tau_{j \ell}(y)}\left[\Phi_{k}(x-y)\right] \nu_{\ell}(y) \nu_{i}(y) e_{i} \odot e_{j} \odot u(y) d \sigma_{y} \\
=\int_{\partial \Omega} \nu_{\ell}(y) \partial_{\tau_{j \ell}(y)}\left[\Phi_{k}(x-y)\right] \nu(y) \odot e_{j} \odot u(y) d \sigma_{y} \\
=-\int_{\partial \Omega}\left(\partial_{y_{j}}-\nu_{j}(y) \partial_{\nu_{y}}\right)\left[\Phi_{k}(x-y)\right] \nu(y) \odot e_{j} \odot u(y) d \sigma_{y} \\
=\int_{\partial \Omega} \partial_{\nu_{y}}\left[\Phi_{k}(x-y)\right] \nu(y) \odot \nu(y) \odot u(y) d \sigma_{y} \\
\quad+\int_{\partial \Omega}\left(\partial_{j} \Phi_{k}\right)(x-y) \nu(y) \odot e_{j} \odot u(y) d \sigma_{y} \\
=-\int_{\partial \Omega} \partial_{\nu_{y}}\left[\Phi_{k}(x-y)\right] u(y) d \sigma_{y}+2 \int_{\partial \Omega} \partial_{\nu_{y}}\left[\Phi_{k}(x-y)\right] u(y) d \sigma_{y} \\
\quad-\int_{\partial \Omega}\left(\partial_{j} \Phi_{k}\right)(x-y) e_{j} \odot \nu(y) \odot u(y) d \sigma_{y} \\
=\int_{\partial \Omega} \partial_{\nu_{y}}\left[\Phi_{k}(x-y)\right] u(y) d \sigma_{y}-\int_{\partial \Omega}\left(\mathbb{D}_{k} \Phi_{k}\right)(x-y) \odot \nu(y) \odot u(y) d \sigma_{y} \\
\quad+k \int_{\partial \Omega} \Phi_{k}(x-y) e_{4} \odot \nu(y) \odot u(y) d \sigma_{y} .
\end{aligned}
$$

Above, the second equality uses (4.43), while the fourth equality makes use of the fact that $\nu \odot \nu=-1$ and $\nu \odot e_{j}=-e_{j} \odot \nu-2 \nu_{j}$ for every $j \in\{1,2,3\}$. In summary, by combining (4.49), (4.52), (4.53) and (4.55) we arrive at the conclusion that

$$
\begin{gathered}
u(x)=\int_{\partial \Omega}\left(\mathbb{D}_{k} \Phi_{k}\right)(x-y) \odot \nu(y) \odot u(y) d \sigma_{y}=\mathscr{C}_{k}\left(\left.\nu \odot u\right|_{\partial \Omega}\right)(x), \\
\text { for } x \text { in } \mathbb{R}^{3} \backslash \bar{\Omega} .
\end{gathered}
$$


With this integral representation in hand, Proposition 4.6 applies and gives that $u$ satisfies (4.41). This reasoning establishes the right-to-left implication in Theorem 4.7. Finally, the opposite implication is a direct consequence of Proposition 4.6 and the decay of the integral kernel of the Cauchy-Clifford operator (compare with CK92, p. 38 or p. 20]). The proof of Theorem 4.7 is therefore finished.

Granted the above analysis, for the remainder of the paper it is natural to make the following definition. Given a bounded domain $\Omega \subset \mathbb{R}^{3}, k \in \mathbb{C} \backslash\{0\}$ with $\operatorname{Im} k \geq 0$, we say that a $k$-monogenic function $u: \Omega_{-} \rightarrow \mathcal{C}_{4}$ decays at infinity if $u(x)=O(1)$ as $|x| \rightarrow \infty$ when $\operatorname{Im} k>0$ (in which case, as pointed out earlier, $u$ decays exponentially), and if $u$ satisfies (4.41) when $k \in \mathbb{R} \backslash\{0\}$.

\section{The invertibility of CAuChy type OPERATORS}

Here we are concerned with the invertibility properties of boundary Cauchy type operators of the form $\lambda I+\nu \wedge C_{k}$ and $\lambda I+\nu \vee C_{k}$, where $\lambda$ is a real parameter and $I$ denotes the identity, on appropriate function spaces. Our first main result in this regard reads as follows.

Theorem 5.1. Let $\Omega \subset \mathbb{R}^{3}$ be an arbitrary Lipschitz domain with compact boundary. Then there exists $\varepsilon=\varepsilon(\Omega)>0$ with the following significance. For every $\lambda \in \mathbb{R}$ with $|\lambda| \geq \frac{1}{2}$ there exists an at most countable set of real numbers $\left\{k_{j}\right\}_{j}$ containing 0 and possibly accumulating only at $\pm \infty$, such that whenever $1<p<2+\varepsilon$ and $k \in \mathbb{C} \backslash\left\{k_{j}\right\}_{j}$ has $\operatorname{Im} k \geq 0$, the operator $\lambda I+\nu \wedge C_{k}$ is an isomorphism of $L_{\text {nor }}^{p, d}\left(\partial \Omega, \mathcal{C l}_{4}\right)$.

The proof of this result occupies the bulk of this section. In the first stage, our goal is to show that there exists $\varepsilon>0$ with the property that

$$
\begin{gathered}
\lambda I+\nu \wedge C_{k} \text { is a Fredholm operator with index zero on } L_{n o r}^{p, d}\left(\partial \Omega, C_{4}\right) \\
\text { whenever } k \in \mathbb{C}, 1<p<2+\varepsilon, \text { and } \lambda \in \mathbb{R} \text { satisfies }|\lambda| \geq \frac{1}{2} .
\end{gathered}
$$

In a broad outline, the plan of attack for establishing (5.1) is as follows. In the first stage, we shall deconstruct the operator $\lambda I+\nu \wedge C_{k}$ as in (5.13), emphasizing its various components, and then proceed to show that these components (cf. (5.16)) are Fredholm operators of index zero on appropriate spaces of scalar and vectorvalued functions. This is accomplished in Lemma 5.4, and it is here (cf. Steps 3-4 in the proof of this result) that the need for restricting ourselves to the case $m=3$ arises. More specifically, here we are exploiting certain intertwining identities, which are specific to the case when $m=3$, between the magnetic dipole operator $\lambda I+M_{0}$ (corresponding to wave number $k=0$ ), on the one hand, and the harmonic double layer potential operator treated in [EM04, on the other hand. Once this task has been carried out, the return to the general case when $k \neq 0$ is done using an argument based on the invariance of the index under compact perturbations. This yields (5.1). With this in hand, the invertibility result in Theorem 5.1 then follows from the Analytic Fredholm Theory (relying on the holomorphicity of the operator-valued function $k \mapsto C_{k}$ ) recorded in Theorem [5.8, and the $L^{2}$-energy identities presented in the proof of Lemma 5.7 (this is where the condition $\operatorname{Im} k \geq 0$ originates).

Turning to specifics, given any function $f \in L_{\text {nor }}^{p, d}\left(\partial \Omega, \mathcal{C l}_{4}\right)$, we make use of Theorem 3.5 in order to write $f=F+e_{4} \odot \widetilde{F}$ with $F, \widetilde{F} \in L_{\text {nor }}^{p, d}\left(\partial \Omega, C_{3}\right)$. We will first 
concentrate on the action of the Cauchy operator on $F$. Recall that, in $\Omega$,

$$
\begin{aligned}
\mathscr{C}_{k} F & =\mathbb{D}_{k} \mathscr{S}_{k} F=d \mathscr{S}_{k} F+\delta \mathscr{S}_{k} F+k e_{4} \odot \mathscr{S}_{k} F \\
& =\mathscr{S}_{k}\left(d_{\partial} F\right)+\delta \mathscr{S}_{k} F+k e_{4} \odot \mathscr{S}_{k} F .
\end{aligned}
$$

Restrict $\mathscr{C}_{k} F$ to the boundary (in the non-tangential sense described in (1.6)), then invoke (4.38) along with the jump formulas for $\mathscr{C}_{k}$ and $\delta \mathscr{S}_{k}$ (cf. Lemma 4.4 and Theorem 4.5), and then finally apply $\nu \wedge$ to both sides of the resulting equality. In this manner, we arrive at the conclusion that, on $\partial \Omega$,

$$
\begin{aligned}
\nu \wedge\left(-\frac{1}{2} \nu \odot F+C_{k} F\right)= & \nu \wedge S_{k}\left(d_{\partial} F\right)+\nu \wedge\left(\frac{1}{2} \nu \vee F+\delta S_{k} F\right) \\
& -k e_{4} \odot\left(\nu \wedge S_{k} F\right) .
\end{aligned}
$$

We may further simplify formula (5.3) by writing

$$
\nu \wedge C_{k} F=\nu \wedge S_{k}\left(d_{\partial} F\right)+\nu \wedge \delta S_{k} F-k e_{4} \odot\left(\nu \wedge S_{k} F\right) \text { on } \partial \Omega .
$$

At this point, we are going to express $F$ as in (3.17) and monitor how the middle term on the right-hand side of (5.4) transforms in the process. For starters, we have

$$
\nu \wedge \delta S_{k} F=\nu \wedge \delta S_{k}\left(\nu f_{0}\right)+\nu \wedge \delta S_{k}\left(* f_{1}\right)+\nu \wedge \delta S_{k}\left(* f_{0}^{\prime}\right),
$$

and the first term on the right-hand side of (5.5) can be rewritten as

$$
\begin{aligned}
\nu \wedge \delta S_{k}\left(\nu f_{0}\right) & =-\nu \wedge\left(\text { p.v. } \int_{\partial \Omega}\left\langle\left(\nabla \Phi_{k}\right)(\cdot-y), \nu(y)\right\rangle f_{0}(y) d \sigma_{y}\right) \\
& =\nu K_{k} f_{0} .
\end{aligned}
$$

For the second term on the right-hand side of (5.5), we make the observation that

$$
\nu \wedge \delta S_{k}\left(* f_{1}\right)=* M_{k} f_{1} .
$$

Finally, we can rewrite the third term on the right-hand side of (5.5) as

$$
\begin{aligned}
\nu \wedge \delta S_{k}\left(* f_{0}^{\prime}\right) & =-*\left(\nu \vee d S_{k} f_{0}^{\prime}\right)=-*\left\langle\nu, \nabla S_{k} f_{0}^{\prime}\right\rangle=-* \partial_{\nu} S_{k} f_{0}^{\prime} \\
& =-* K_{k}^{\top} f_{0}^{\prime} .
\end{aligned}
$$

Hence, in light of these identifications, (5.4) becomes

$$
\begin{aligned}
\nu \wedge C_{k} F= & \nu K_{k} f_{0}+* M_{k} f_{1}-* K_{k}^{\top} f_{0}^{\prime}+\nu \wedge S_{k}\left(d_{\partial} F\right) \\
& -k e_{4} \odot\left(\nu \wedge S_{k} F\right) .
\end{aligned}
$$

To proceed, let $I$ denote the identity operator. Then, for every $\lambda \in \mathbb{R}$, (5.9) allows us to write

$$
\begin{aligned}
\left(\lambda I+\nu \wedge C_{k}\right) F= & \nu\left[\lambda I+K_{k}\right] f_{0}+*\left[\lambda I+M_{k}\right] f_{1}+*\left[\lambda I-K_{k}^{\top}\right] f_{0}^{\prime} \\
& +\nu \wedge S_{k}\left(d_{\partial} F\right)-k e_{4} \odot\left(\nu \wedge S_{k} F\right) .
\end{aligned}
$$

Similarly to the treatment of the function $F$, decomposing $\widetilde{F}=\nu \widetilde{f}_{0}+* \widetilde{f}_{1}+* \widetilde{f}_{0}^{\prime}$ yields an analogous identity to (5.10) (formally obtained by placing a tilde on all functions involved). We are now ready to compute $\left(\lambda I+\nu \wedge C_{k}\right) f$. First, since based on Clifford calculus one can check that

$$
\left(\nu \wedge C_{k}\right) e_{4} \odot=e_{4} \odot\left(\nu \wedge C_{k}\right)-2 k e_{4} \odot\left(\nu \wedge S_{k}\right),
$$


we may write

$$
\begin{aligned}
\left(\lambda I+\nu \wedge C_{k}\right) f= & \left(\lambda I+\nu \wedge C_{k}\right) F+e_{4} \odot\left(\lambda I+\nu \wedge C_{k}\right) \widetilde{F} \\
& -2 k e_{4} \odot\left(\nu \wedge S_{k} \widetilde{F}\right) .
\end{aligned}
$$

By using (5.10) in its original format, as well as its version for $\widetilde{F}$, we may then finally express $\left(\lambda I+\nu \wedge C_{k}\right) f$ in the form

$$
\begin{aligned}
\left(\lambda I+\nu \wedge C_{k}\right) f= & \nu\left[\lambda I+K_{k}\right] f_{0}+*\left[\lambda I+M_{k}\right] f_{1}+*\left[\lambda I-K_{k}^{\top}\right] f_{0}^{\prime} \\
& +\nu \wedge S_{k}\left(d_{\partial} F\right)-k e_{4} \odot\left(\nu \wedge S_{k} F\right) \\
& +e_{4} \odot\left\{\nu\left[\lambda I+K_{k}\right] \widetilde{f}_{0}+*\left[\lambda I+M_{k}\right] \tilde{f}_{1}+*\left[\lambda I-K_{k}^{\top}\right] \tilde{f}_{0}^{\prime}\right. \\
& \left.+\nu \wedge S_{k}\left(d_{\partial} \widetilde{F}\right)-k e_{4} \odot \nu\left(\wedge S_{k} \widetilde{F}\right)\right\} .
\end{aligned}
$$

In order to make the task of managing the expression on the right-hand side of the above equality easier, let us introduce the following isomorphism:

$$
T: L_{\text {nor }}^{p, d}\left(\partial \Omega, \mathcal{C l}_{4}\right) \rightarrow
$$

$$
L_{1}^{p}(\partial \Omega) \oplus L_{\text {tan }}^{p, \text { Div }}(\partial \Omega) \oplus L^{p}(\partial \Omega) \oplus L_{1}^{p}(\partial \Omega) \oplus L_{\text {tan }}^{p, \text { Div }}(\partial \Omega) \oplus L^{p}(\partial \Omega)
$$

defined by

$$
T(f):=\left(f_{0}, f_{1}, f_{0}^{\prime}, \tilde{f}_{0}, \tilde{f}_{1}, \tilde{f}_{0}^{\prime}\right) \quad \text { whenever } f \text { is as in (3.28). }
$$

In addition, let us consider an operator $\widetilde{Q}$ which, in matrix form, is given by the $6 \times 6$ matrix

$$
\widetilde{Q}:=\left[\begin{array}{cccccc}
a & 0 & 0 & 0 & 0 & 0 \\
0 & b & 0 & 0 & 0 & 0 \\
0 & 0 & c & 0 & 0 & 0 \\
0 & 0 & 0 & a & 0 & 0 \\
0 & 0 & 0 & 0 & b & 0 \\
0 & 0 & 0 & 0 & 0 & c
\end{array}\right]
$$

where

$$
a:=\lambda I+K_{k}, b:=\lambda I+M_{k} \quad \text { and } \quad c:=\lambda I-K_{k}^{\top} .
$$

The immediate goal is to show that, when considered between appropriate spaces, the operator $\widetilde{Q}$ is Fredholm with index zero. As a preamble, we record some functional analytical results from [MMP97].

Lemma 5.2. Let $X, Y, Z$ be Banach spaces and consider the commutative diagram

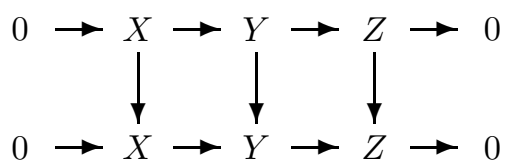

where all arrows are linear and bounded operators and the horizontal sequences are exact. Then the following claims are valid:

(1) If two vertical arrows are isomorphisms, then so is the third one. 
(2) If two vertical arrows are Fredholm operators, then so is the third. Moreover, the index of the middle vertical arrow is the sum of the indexes of the other two vertical arrows.

Lemma 5.3. Let $X_{0}, Y_{0}, X_{1}, Y_{1}$ be Banach spaces and assume that the following diagram is commutative:

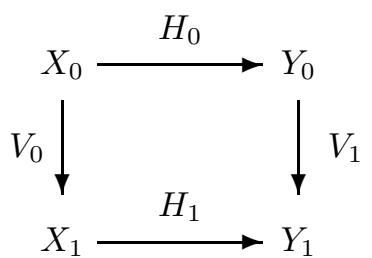

If three of the four arrows are Fredholm operators, then all arrows are Fredholm operators and

$$
\text { index } H_{0}-\operatorname{index} H_{1}=\operatorname{index} V_{0}-\operatorname{index} V_{1} \text {. }
$$

Let us state our earlier claim about the operator $\widetilde{Q}$ in a more pedantic fashion.

Lemma 5.4. Suppose that $\Omega \subset \mathbb{R}^{3}$ is a Lipschitz domain with compact boundary. Then there exists $\varepsilon>0$ such that for each $p \in(1,2+\varepsilon)$ and each $\lambda \in \mathbb{R}$ with $|\lambda| \geq \frac{1}{2}$ the operator $\widetilde{Q}$ introduced in (5.16) is Fredholm with index zero when acting on

$$
L_{1}^{p}(\partial \Omega) \oplus L_{t a n}^{p, \text { Div }}(\partial \Omega) \oplus L^{p}(\partial \Omega) \oplus L_{1}^{p}(\partial \Omega) \oplus L_{t a n}^{p, \text { Div }}(\partial \Omega) \oplus L^{p}(\partial \Omega) .
$$

Proof. We shall proceed in a series of steps, starting with:

Step 1 . There exists $\varepsilon>0$ such that for each $p \in(1,2+\varepsilon)$ and each $\lambda \in \mathbb{R}$ with $|\lambda| \geq \frac{1}{2}$ the operator $\lambda I+K_{0}^{\top}$ is an isomorphism of $L^{p}(\partial \Omega)$, and the operator $\lambda I+K_{0}$ is an isomorphism of $L_{1}^{p}(\partial \Omega)$. This key ingredient in our approach has been established in EM04.

Step 2. There exists $\varepsilon>0$ such that for each $p \in(1,2+\varepsilon)$, each $k \in \mathbb{C}$ and each $\lambda \in \mathbb{R}$ with $|\lambda| \geq \frac{1}{2}$ the operator $\lambda I+K_{k}^{\top}$ is a Fredholm operator with index zero on $L^{p}(\partial \Omega)$ and $\lambda I+K_{k}$ is a Fredholm operator with index zero on $L_{1}^{p}(\partial \Omega)$. Indeed, we write

$$
\begin{gathered}
\lambda I+K_{k}^{\top}=\lambda I+K_{0}^{\top}+\left(K_{k}^{\top}-K_{0}^{\top}\right) \quad \text { and } \\
\lambda I+K_{k}=\lambda I+K_{0}+\left(K_{k}-K_{0}\right) .
\end{gathered}
$$

The key observation is that, thanks to Lemma 4.1, the integral kernel of the operator $-K_{k}^{\top}+K_{0}^{\top}$ has only a weak singularity and, as such, $-K_{k}^{\top}+K_{0}^{\top}$ is compact on $L^{p}(\partial \Omega)$ for every $p \in(1, \infty)$. Thus, if $p \in(1,2+\varepsilon)$ with $\varepsilon>0$ as in Step 1 , it follows that $\lambda I+K_{k}^{\top}$ is the sum of a compact operator and an invertible operator; hence $\lambda I+K_{k}^{\top}$ is a Fredholm operator with index zero on $L^{p}(\partial \Omega)$. A similar reasoning works in the case of $\lambda I+K_{k}$, using the second decomposition in (5.20) and the fact that, by (4.7), $-K_{k}+K_{0}$ is compact on $L_{1}^{p}(\partial \Omega)$ for each $p \in(1, \infty)$. The desired conclusion follows.

Step 3. There exists $\varepsilon>0$ such that for each $p \in(1,2+\varepsilon)$ and each $\lambda \in \mathbb{R}$ with $|\lambda| \geq \frac{1}{2}$ the operator $\lambda I+M_{0}$ is Fredholm with index zero on $L_{t a n}^{p, 0}(\partial \Omega)$.

The proof of this step requires some results from MMP97 and Mi95 which we now recall. First, by [MMP97, (5.12)], we have $\left(\nu \times \nabla_{t a n}\right) K_{0}=M_{0}\left(\nu \times \nabla_{t a n}\right)$ 
and, by [MMP97, Theorem 5.1-(v)], if $1<p<\infty$, then $\nu \times \nabla_{\text {tan }}$ is a Fredholm operator with index zero when acting from $L_{1}^{p}(\partial \Omega)$ into $L_{\text {tan }}^{p, 0}(\partial \Omega)$ (recall that the latter space has been defined in (3.7)). As a consequence, we have the following commutative diagram:

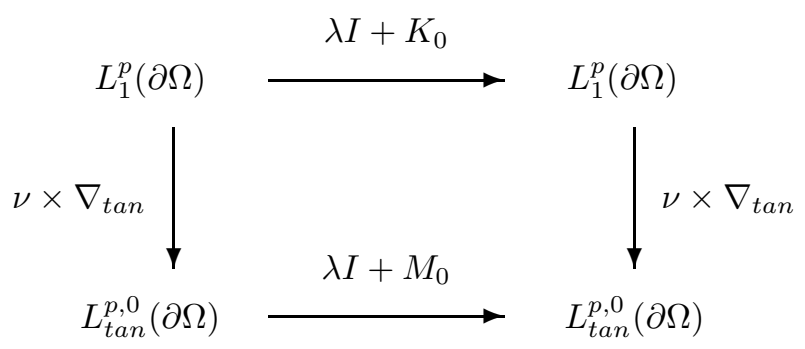

where the two vertical arrows are Fredholm with index zero. Since, by Step 1, the operator $\lambda I+K_{0}$ is an isomorphism in the context of the diagram if $1<p<2+\varepsilon$ (with $\varepsilon>0$ as in Step 1), it follows from Lemma 5.3 that $\lambda I+M_{0}$ is Fredholm with index zero on $L_{t a n}^{p, 0}(\partial \Omega)$ whenever $\lambda \in \mathbb{R}$ satisfies $|\lambda| \geq \frac{1}{2}$ and $1<p<2+\varepsilon$.

Step 4. There exists $\varepsilon>0$ such that for each $p \in(1,2+\varepsilon)$ and each $\lambda \in \mathbb{R}$ with $|\lambda| \geq \frac{1}{2}$ the operator $\lambda I+M_{0}$ is Fredholm with index zero when acting on the quotient space on $L_{\text {tan }}^{p, \operatorname{Div}}(\partial \Omega) / L_{\text {tan }}^{p, 0}(\partial \Omega)$.

From [MMP97, Theorem 5.1-(viii)] we know that the surface divergence operator, Div, is Fredholm when acting from the quotient space $L_{t a n}^{p \text {, Div }}(\partial \Omega) / L_{\text {tan }}^{p, 0}(\partial \Omega)$ into $L^{p}(\partial \Omega)$, for every $p \in(1, \infty)$. Specializing [MMP97, Lemma 4.4] to the case when $k=0$ then gives the following commutative diagram:



We may then proceed as in Step 3 in order to arrive at the desired conclusion.

Step 5. There exists $\varepsilon>0$ such that for each $p \in(1,2+\varepsilon)$ and each $\lambda \in \mathbb{R}$ with $|\lambda| \geq \frac{1}{2}$ the operator $\lambda I+M_{0}$ is Fredholm with index zero when acting on $L_{\text {tan }}^{p \text {,Div }}(\partial \Omega)$.

The idea is to use Lemma 5.2 with

$$
X:=L_{t a n}^{p, 0}(\partial \Omega), \quad Y:=L_{t a n}^{p, \operatorname{Div}}(\partial \Omega), \quad Z:=L_{t a n}^{p, \text { Div }}(\partial \Omega) / L_{t a n}^{p, 0}(\partial \Omega),
$$

and where, in each exact sequence, the second horizontal arrow is the inclusion operator, the third horizontal arrow is the projection operator and each vertical arrow is the operator $\lambda I+M_{0}$. By Steps 3-4, the first and the third vertical arrows in the diagram from Lemma 5.2 are both Fredholm operators with index zero, so the desired conclusion follows.

Step 6. There exists $\varepsilon>0$ such that for each $p \in(1,2+\varepsilon)$, each $k \in \mathbb{C}$, and each $\lambda \in \mathbb{R}$ with $|\lambda| \geq \frac{1}{2}$ the operator $\lambda I+M_{k}$ is Fredholm with index zero when acting on 
$L_{\text {tan }}^{p \text {,Div }}(\partial \Omega)$. Much as in the treatment of Step 2, we rewrite the operator $\lambda I+M_{k}$ in the form

$$
\lambda I+M_{k}=\lambda I+M_{0}+\left(M_{k}-M_{0}\right),
$$

and observe that by Lemma 4.1 (cf. (4.7) in particular) the difference $M_{k}-M_{0}$ is a compact operator on $L_{t a n}^{p \text {,Div }}(\partial \Omega)$ for any $p \in(1, \infty)$. The endgame is then as before.

Step 7. There exists $\varepsilon>0$ such that for each $p \in(1,2+\varepsilon)$, each $k \in \mathbb{C}$, and each $\lambda \in \mathbb{R}$ with $|\lambda| \geq \frac{1}{2}$ the operator $\widetilde{Q}$ is Fredholm with index zero acting on the space (5.19). This follows readily from Steps 1-6 and the structure of $\widetilde{Q}$ (see (5.16)).

Having introduced and studied the operator $\widetilde{Q}$, we now consider

$$
Q:=T^{-1} \circ \widetilde{Q} \circ T \text {. }
$$

Since the operator $T$ is an isomorphism and $\widetilde{Q}$ is Fredholm with index zero, $Q$ is a Fredholm operator with index zero on $L_{\text {nor }}^{p, d}\left(\partial \Omega, \mathcal{C l}_{4}\right)$ whenever $k \in \mathbb{C}, \lambda \in \mathbb{R}$ with $|\lambda| \geq \frac{1}{2}$ and $1<p<2+\varepsilon$. In order to continue the proof, we consider two operators acting on an arbitrary $F \in L_{\text {nor }}^{p, d}\left(\partial \Omega, \mathcal{C l}_{3}\right)$ as follows:

$$
R_{1}(F):=\nu \wedge S_{k}\left(d_{\partial} F\right), \quad R_{2}(F):=-k \nu \wedge S_{k}(F) .
$$

Lemma 5.5. Let $\Omega \subset \mathbb{R}^{3}$ be a Lipschitz domain with compact boundary. Then the operators $R_{j}, 1 \leq j \leq 2$, introduced above are compact when acting from the space $L_{\text {nor }}^{p, d}\left(\partial \Omega, \mathcal{C l}_{3}\right)$ into itself, for any $p \in(1, \infty)$ and any $k \in \mathbb{C}$.

In order to prove this lemma, we single out a simple but useful functional analytic result.

Lemma 5.6. Assume that $\Omega \subset \mathbb{R}^{3}$ is a Lipschitz domain with compact boundary and suppose that $X$ is a given Banach space. Let $T$ be a linear, bounded operator from $X$ into $L_{n o r}^{p, d}\left(\partial \Omega, \mathcal{C}_{3}\right)$ and denote by $\iota$ the canonical inclusion of $L_{\text {nor }}^{p, d}\left(\partial \Omega, \mathcal{C l}_{3}\right)$ into $L^{p}\left(\partial \Omega, \mathcal{C}_{3}\right)$. Then $T$ is compact if and only if $\iota \circ T: X \rightarrow L^{p}\left(\partial \Omega, \mathcal{C}_{3}\right)$ and $d_{\partial} \circ T: X \rightarrow L^{p}\left(\partial \Omega, C_{3}\right)$ are compact operators.

Proof. In one direction, if $T$ is compact then, clearly, $\iota \circ T$ and $d_{\partial} \circ T$ are compact. Conversely, assume that $\iota \circ T$ and $d_{\partial} \circ T$ are compact, and consider a bounded sequence $\left\{x_{j}\right\}_{j}$ in $X$. Then there exists a subsequence $\left\{x_{j_{k}}\right\}_{k}$ such that $\left\{T x_{j_{k}}\right\}_{k}$ converges in $L^{p}\left(\partial \Omega, \mathcal{C}_{3}\right)$ and $\left\{d_{\partial}\left(T x_{j_{k}}\right)\right\}_{k}$ converges in $L^{p}\left(\partial \Omega, \mathcal{C}_{3}\right)$. Hence, $\left\{T x_{j_{k}}\right\}_{k}$ converges in $L_{\text {nor }}^{p, d}\left(\partial \Omega, \mathcal{C l}_{3}\right)$, proving that $T: X \rightarrow L_{\text {nor }}^{p, d}\left(\partial \Omega, \mathcal{C l}_{3}\right)$ is compact.

We are now ready to present the

Proof of Lemma 5.5. Since the operator $\nu \wedge S_{k}$ is compact from $L^{p}\left(\partial \Omega, \mathcal{C}_{3}\right)$ into itself and since the operator $d_{\partial}$ is bounded from $L_{\text {nor }}^{p, d}\left(\partial \Omega, \mathcal{C l}_{3}\right)$ into $L^{p}\left(\partial \Omega, \mathcal{C l}_{3}\right)$, the operator $R_{1}$ is compact from $L_{n o r}^{p, d}\left(\partial \Omega, C_{3}\right)$ into $L^{p}\left(\partial \Omega, C_{3}\right)$. Moreover, based on Lemma 3.2, (4.22) and Lemma 3.1, we may write

$$
d_{\partial}\left(R_{1} F\right)=d_{\partial}\left(\nu \wedge S_{k}\left(d_{\partial} F\right)\right)=-\nu \wedge d\left(S_{k}\left(d_{\partial} F\right)\right)=\nu \wedge S_{k}\left(d_{\partial}^{2} F\right)=0 .
$$

Hence, obviously, $d_{\partial} R_{1}$ is a compact operator from $L_{\text {nor }}^{p, d}\left(\partial \Omega, \mathcal{C}_{3}\right)$ into $L^{p}\left(\partial \Omega, \mathcal{C}_{3}\right)$. Then by Lemma [5.6] we may conclude that $R_{1}$ is compact from $L_{\text {nor }}^{p, d}\left(\partial \Omega, C_{3}\right)$ into itself. 
We next consider the operator $R_{2}$. First, by the compactness of $S_{k}$ on $L^{p}(\partial \Omega)$, we conclude that $R_{2}$ is a compact operator from $L_{n o r}^{p, d}\left(\partial \Omega, \mathcal{C}_{3}\right)$ into $L^{p}\left(\partial \Omega, \mathcal{C}_{3}\right)$. On the other hand,

$$
d_{\partial}\left(R_{2} F\right)=d_{\partial}\left(k \nu \wedge S_{k} F\right)=-k \nu \wedge d\left(S_{k} F\right)=k \nu \wedge S_{k}\left(d_{\partial} F\right),
$$

thanks to Lemma 3.2 and (4.22). Since $\nu \wedge S_{k}$ is compact from $L^{p}\left(\partial \Omega, \mathcal{C}_{3}\right)$ into itself and since the operator $d_{\partial}$ is bounded from $L_{\text {nor }}^{p, d}\left(\partial \Omega, \mathcal{C}_{3}\right)$ into $L^{p}\left(\partial \Omega, \mathcal{C l}_{3}\right)$, we infer that $d_{\partial} R_{2}$ is a compact operator from $L_{\text {nor }}^{p, d}\left(\partial \Omega, \mathcal{C}_{3}\right)$ into $L^{p}\left(\partial \Omega, \mathcal{C}_{3}\right)$. Once again, by applying Lemma 5.6. we may conclude that $R_{2}$ is a compact operator from $L_{\text {nor }}^{p, d}\left(\partial \Omega, C_{3}\right)$ into itself. This concludes the proof of the lemma.

Having dealt with the operators $R_{j}, 1 \leq j \leq 2$, we wish to glue them together using two projection operators, namely

$$
\begin{gathered}
\pi, \widetilde{\pi}: L_{\text {nor }}^{p, d}\left(\partial \Omega, \mathcal{C}_{4}\right) \longrightarrow L_{\text {nor }}^{p, d}\left(\partial \Omega, \mathcal{C l}_{3}\right) \quad \text { defined by } \\
\pi(f):=F \text { and } \widetilde{\pi}(f):=\widetilde{F} \text {, if } f=F+e_{4} \odot \widetilde{F} \text { is as in (3.27). }
\end{gathered}
$$

We then set

$$
R:=R_{1} \circ \pi+e_{4} \odot R_{2} \circ \pi+e_{4} \odot R_{1} \circ \tilde{\pi}-R_{2} \circ \tilde{\pi} .
$$

Since $\pi$ and $\widetilde{\pi}$ are bounded and since, by Lemma 5.5 , the $R_{j}$ 's are compact, it follows that $R$ is a compact operator from $L_{\text {nor }}^{p, d}\left(\partial \Omega, \mathcal{C}_{4}\right)$ into itself. From the definition of the operator $Q$ in (5.23), the definition of the operator $R$ in (5.28), and equality (5.13), one can easily check that

$$
\lambda I+\nu \wedge C_{k}=Q+R .
$$

This identity, highlighting the structure of $\lambda I+\nu \wedge C_{k}$, is the reason for which the operators $Q$ and $R$ have been introduced and studied. In particular, since $Q$ is a Fredholm operator with index zero on $L_{\text {nor }}^{p, d}\left(\partial \Omega, \mathcal{C l}_{4}\right)$ and $R$ is compact on the same space, it follows from (5.29) that the operator $\lambda I+\nu \wedge C_{k}$ is Fredholm with index zero on $L_{\text {nor }}^{p, d}\left(\partial \Omega, \mathcal{C l}_{4}\right)$ granted that $k \in \mathbb{C}, \lambda \in \mathbb{R}$ satisfies $|\lambda| \geq \frac{1}{2}$, and $1<p<2+\varepsilon$. This concludes the proof of (5.1).

Establishing the Fredholmness of $\lambda I+\nu \wedge C_{k}$ (in the context of (5.1)) is a key step in the proof of Theorem 5.1. Note that, for this particular result, all complex values $k \in \mathbb{C}$ are allowed. It is only when dealing with invertibility issues that certain exceptional real values of the parameter $k$ must (necessarily) be excluded. It is therefore natural that we first study the invertibility of $\lambda I+\nu \wedge C_{k}$ when the complex number $k$ lies in the open upper half-plane. For the special case when $p=2$ this is done in the lemma below.

Lemma 5.7. Let $\Omega \subset \mathbb{R}^{3}$ be a Lipschitz domain with compact boundary. Then for each $k \in \mathbb{C}$ with $\operatorname{Im} k>0$ and each $\lambda \in \mathbb{R}$ with $|\lambda| \geq \frac{1}{2}$, the operator $\lambda I+\nu \wedge C_{k}$ is an isomorphism when acting from $L_{\text {nor }}^{2, d}\left(\partial \Omega, \mathcal{C l}_{4}\right)$ into itself.

Proof. From (5.1), we already know that the operator in question is Fredholm with index zero on $L_{\text {nor }}^{2, d}\left(\partial \Omega, \mathcal{C l}_{4}\right)$, so we only need to show that this operator is injective whenever $\operatorname{Im} k>0$. Fix such a number $k$, and assume that $\lambda \in \mathbb{R}$ satisfies $|\lambda| \geq \frac{1}{2}$. Also, let $f \in L_{\text {nor }}^{2, d}\left(\partial \Omega, \mathcal{C l}_{4}\right)$ be such that

$$
\lambda f+\nu \wedge C_{k} f=0 \quad \text { on } \partial \Omega .
$$

Our long-term goal is to show that $f=0$. With this in mind, assume that the real parameter $\lambda$ satisfies $\lambda \geq \frac{1}{2}$. Next, recall (1.1) and introduce $u^{ \pm}:=\mathscr{C}_{k} f$ in $\Omega_{ \pm}$. 
The objective is to eventually show that both $u^{+}$and $u^{-}$vanish identically. For the time being, we note that

$$
\left.u^{ \pm}\right|_{\partial \Omega}=\mp \frac{1}{2} \nu \odot f+C_{k} f= \pm \frac{1}{2} \nu \vee f+C_{k} f \quad \text { on } \partial \Omega .
$$

Therefore, by (1) in Lemma 2.1,

$$
\nu \vee u^{+}-\nu \vee u^{-}=0 \quad \text { on } \partial \Omega
$$

and, by (2.10) and the normality of $f$, for any $\mu \in \mathbb{R}$ we have

$$
\begin{aligned}
\nu \wedge u^{+}-\mu \nu \wedge u^{-} & =\frac{1}{2}(\mu+1) f+(1-\mu) \nu \wedge C_{k} f \\
& =(1-\mu)\left[\frac{1}{2}\left(\frac{1+\mu}{1-\mu}\right) f+\nu \wedge C_{k} f\right] \quad \text { on } \partial \Omega
\end{aligned}
$$

Specializing (5.33) to the choice

$$
\mu:=\frac{2 \lambda-1}{2 \lambda+1} \geq 0
$$

yields, due to (5.30), that

$$
\nu \wedge u^{+}-\mu \nu \wedge u^{-}=0 \quad \text { on } \partial \Omega .
$$

Applying the operator $\delta_{\partial}$ to both sides of the equality (5.32) gives, due to (3.15),

$$
\nu \vee \delta u^{+}=\nu \vee \delta u^{-} \quad \text { on } \partial \Omega .
$$

Similarly, applying the operator $d_{\partial}$ to both sides of the equality (5.35) gives

$$
\nu \wedge d u^{+}=\mu \nu \wedge d u^{-} \quad \text { on } \partial \Omega .
$$

Recall that $\nu$ stands for the outward unit normal to the boundary of $\Omega=\Omega_{+}$, and define

$$
\nu_{+}:=\nu \quad \text { and } \quad \nu_{-}:=-\nu \text {. }
$$

Let us now compute the integral $\int_{\Omega_{+}}\left|d u^{+}\right|^{2} d x$. First, by definition of the inner product and since $d u^{+}=-\delta u^{+}-k e_{4} \odot u^{+}$, we obtain

$$
\begin{aligned}
\int_{\Omega_{+}}\left|d u^{+}\right|^{2} d x & =\int_{\Omega_{+}}\left\langle d u^{+},\left(d u^{+}\right)^{c}\right\rangle d x \\
& =-\int_{\Omega_{+}}\left\langle\delta u^{+},\left(d u^{+}\right)^{c}\right\rangle d x-\int_{\Omega_{+}}\left\langle k e_{4} \odot u^{+},\left(d u^{+}\right)^{c}\right\rangle d x .
\end{aligned}
$$

An integration by parts (cf. Lemma 2.4) gives, due to the fact that $d^{2}=0$,

$$
\int_{\Omega_{+}}\left\langle\delta u^{+},\left(d u^{+}\right)^{c}\right\rangle d x=-\int_{\partial \Omega}\left\langle\nu_{+} \vee u^{+},\left(d u^{+}\right)^{c}\right\rangle d \sigma .
$$

Next using property (7) in Lemma 2.1 together with (5.37) and (5.36) yields, after some algebra,

$$
\int_{\partial \Omega}\left\langle\nu_{+} \vee u^{+},\left(d u^{+}\right)^{c}\right\rangle d \sigma=-\mu \int_{\partial \Omega}\left\langle\nu_{+} \vee u^{-},\left(d u^{-}\right)^{c}\right\rangle d \sigma .
$$

In concert, formulas (5.39)-(5.41) show that

$$
\int_{\Omega_{+}}\left|d u^{+}\right|^{2} d x=-\mu \int_{\partial \Omega}\left\langle\nu_{+} \vee u^{-},\left(d u^{-}\right)^{c}\right\rangle d \sigma-\int_{\Omega_{+}}\left\langle k e_{4} \odot u^{+},\left(d u^{+}\right)^{c}\right\rangle d x .
$$


A similar computation, emphasizing $\delta$ in place of $d$, also yields

$$
\begin{aligned}
\int_{\Omega_{+}}\left|\delta u^{+}\right|^{2} d x= & -\mu \int_{\partial \Omega}\left\langle\nu_{+} \wedge u^{-},\left(\delta u^{-}\right)^{c}\right\rangle d \sigma \\
& -\int_{\Omega_{+}}\left\langle k e_{4} \odot u^{+},\left(\delta u^{+}\right)^{c}\right\rangle d x .
\end{aligned}
$$

The above considerations pertain to the interior domain $\Omega_{+}$, and we are now interested in computing $\mu \int_{\Omega_{-}}\left|d u^{-}\right|^{2} d x$ and $\mu \int_{\Omega_{-}}\left|\delta u^{-}\right|^{2} d x$. Following the same pattern (and recalling that the function $u^{-}$, along with any of its derivatives, decays exponentially at infinity in the current case), we obtain

$$
\begin{aligned}
\mu \int_{\Omega_{-}}\left|d u^{-}\right|^{2} d x= & \mu \int_{\partial \Omega}\left\langle\nu_{+} \vee u^{-},\left(d u^{-}\right)^{c}\right\rangle d \sigma \\
& -\mu \int_{\Omega_{-}}\left\langle k e_{4} \odot u^{-},\left(d u^{-}\right)^{c}\right\rangle d x
\end{aligned}
$$

and

$$
\begin{aligned}
\mu \int_{\Omega_{-}}\left|\delta u^{-}\right|^{2} d x= & \mu \int_{\partial \Omega}\left\langle\nu_{+} \wedge u^{-},\left(\delta u^{-}\right)^{c}\right\rangle d \sigma \\
& -\mu \int_{\Omega_{-}}\left\langle k e_{4} \odot u^{-},\left(\delta u^{-}\right)^{c}\right\rangle d x .
\end{aligned}
$$

Summing up formulas (5.42), (5.43), (5.44) and (5.45) and performing a number of obvious cancellations, we finally obtain (also using the fact that $\left|e_{4} \odot u^{ \pm}\right|=\left|u^{ \pm}\right|$)

$$
\begin{aligned}
\int_{\Omega_{+}}\left(\left|d u^{+}\right|^{2}+\left|\delta u^{+}\right|^{2}\right) d x & +\mu \int_{\Omega_{-}}\left(\left|d u^{-}\right|^{2}+\left|\delta u^{-}\right|^{2}\right) d x \\
& =|k|^{2}\left(\int_{\Omega_{+}}\left|u^{+}\right|^{2} d x+\mu \int_{\Omega_{-}}\left|u^{-}\right|^{2} d x\right) .
\end{aligned}
$$

On the other hand, based on Lemma 2.4 we may write

$$
\begin{aligned}
\int_{\Omega_{+}}\left|d u^{+}\right|^{2} d x & =\int_{\Omega_{+}}\left\langle d u^{+},\left(d u^{+}\right)^{c}\right\rangle d x \\
& =\int_{\Omega_{+}}\left\langle u^{+},\left(\delta d u^{+}\right)^{c}\right\rangle d x+\int_{\partial \Omega}\left\langle\nu_{+} \wedge u^{+},\left(d u^{+}\right)^{c}\right\rangle d \sigma
\end{aligned}
$$

and

$$
\begin{aligned}
\int_{\Omega_{+}}\left|\delta u^{+}\right|^{2} d x & =\int_{\Omega_{+}}\left\langle\delta u^{+},\left(\delta u^{+}\right)^{c}\right\rangle d x \\
& =\int_{\Omega_{+}}\left\langle u^{+},\left(d \delta u^{+}\right)^{c}\right\rangle d x-\int_{\partial \Omega}\left\langle\nu_{+} \vee u^{+},\left(\delta u^{+}\right)^{c}\right\rangle d \sigma
\end{aligned}
$$


Summing up (5.47)-(5.48) and using the fact that $(\delta d+d \delta) u^{+}=-\Delta u^{+}=k^{2} u^{+}$in $\Omega_{+}$therefore gives

$$
\begin{aligned}
\int_{\Omega_{+}}\left|d u^{+}\right|^{2} d x & +\int_{\Omega_{+}}\left|\delta u^{+}\right|^{2} d x-\int_{\Omega_{+}}\left(k^{2}\right)^{c}\left|u^{+}\right|^{2} d x \\
& =\int_{\partial \Omega}\left\langle\nu_{+} \wedge u^{+},\left(d u^{+}\right)^{c}\right\rangle d \sigma-\int_{\partial \Omega}\left\langle\nu_{+} \vee u^{+},\left(\delta u^{+}\right)^{c}\right\rangle d \sigma .
\end{aligned}
$$

Strictly speaking, since we are integrating second-order derivatives of $u^{+}$in $\Omega_{+}$, in order to prove (5.49) one would need to approximate $\Omega$ by a nested sequence of relatively compact subdomains $\left\{\Omega_{j}\right\}_{j}$ of $\Omega$ with the property that $\Omega_{j} \nearrow \Omega$ as $j \rightarrow \infty$, in the precise manner described in [Ve84, then derive a version of (5.49) in each $\Omega_{j}$ and, finally, passing to the limit in $j$ in order to arrive at (5.49). The details of this matter are, however, well understood by now, so we omit the details.

Next, based on the fact that $d u^{+}=-\delta u^{+}-k e_{4} \odot u^{+}$, (5.35), (5.37), (5.38), (5.36) and (5.32), we may successively transform the first boundary integral on the right-hand side of (5.49) as follows:

$$
\begin{aligned}
\int_{\partial \Omega}\left\langle\nu_{+} \wedge u^{+},\left(d u^{+}\right)^{c}\right\rangle d \sigma \\
=-\int_{\partial \Omega}\left\langle\nu_{+} \wedge u^{+},\left(\delta u^{+}\right)^{c}\right\rangle d \sigma-\int_{\partial \Omega}\left\langle\nu_{+} \wedge u^{+},\left(k e_{4} \odot u^{+}\right)^{c}\right\rangle d \sigma \\
\quad=-\int_{\partial \Omega}\left\langle u_{+}, \nu_{+} \vee\left(\delta u^{+}\right)^{c}\right\rangle d \sigma-\int_{\partial \Omega}\left\langle u_{+}, \nu_{+} \vee\left(k e_{4} \odot u^{+}\right)^{c}\right\rangle d \sigma \\
=-\mu \int_{\partial \Omega}\left\langle\nu_{+} \wedge u^{-},\left(\delta u^{-}\right)^{c}\right\rangle d \sigma-\mu \int_{\partial \Omega}\left\langle\nu_{+} \wedge u^{-},\left(k e_{4} \odot u^{-}\right)^{c}\right\rangle d \sigma \\
=\mu \int_{\partial \Omega}\left\langle\nu_{-} \wedge u^{-},\left(\delta u^{-}\right)^{c}\right\rangle d \sigma+\mu \int_{\partial \Omega}\left\langle\nu_{-} \wedge u^{-},\left(k e_{4} \odot u^{-}\right)^{c}\right\rangle d \sigma .
\end{aligned}
$$

Similarly, the second boundary integral on the right-hand side of (5.49) can be transformed into

$$
\begin{aligned}
\int_{\partial \Omega}\left\langle\nu_{+} \vee u^{+},\left(\delta u^{+}\right)^{c}\right\rangle d \sigma= & \mu \int_{\partial \Omega}\left\langle\nu_{-} \vee u^{-},\left(d u^{-}\right)^{c}\right\rangle d \sigma \\
& +\mu \int_{\partial \Omega}\left\langle\nu_{-} \vee u^{-},\left(k e_{4} \odot u^{-}\right)^{c}\right\rangle d \sigma .
\end{aligned}
$$

Hence, all together, from (5.49)-(5.51) we obtain

$$
\begin{array}{rl}
\int_{\Omega_{+}}\left|d u^{+}\right|^{2} & d x+\int_{\Omega_{+}}\left|\delta u^{+}\right|^{2} d x-\int_{\Omega_{+}}\left(k^{2}\right)^{c}\left|u^{+}\right|^{2} d x \\
& =\mu \int_{\partial \Omega}\left\langle\nu_{-} \wedge u^{-},\left(\delta u^{-}\right)^{c}\right\rangle d \sigma+\mu \int_{\partial \Omega}\left\langle\nu_{-} \wedge u^{-},\left(k e_{4} \odot u^{-}\right)^{c}\right\rangle d \sigma \\
& -\mu \int_{\partial \Omega}\left\langle\nu_{-} \vee u^{-},\left(d u^{-}\right)^{c}\right\rangle d \sigma-\mu \int_{\partial \Omega}\left\langle\nu_{-} \vee u^{-},\left(k e_{4} \odot u^{-}\right)^{c}\right\rangle d \sigma
\end{array}
$$

A similar analysis can be carried out in $\Omega_{-}$. Specifically, we have

$$
\begin{aligned}
\mu \int_{\Omega_{-}}\left|d u^{-}\right|^{2} d x+\mu \int_{\Omega_{-}}\left|\delta u^{-}\right|^{2} d x-\mu \int_{\Omega_{-}}\left(k^{2}\right)^{c}\left|u^{-}\right|^{2} d x \\
\quad=-\mu \int_{\partial \Omega}\left\langle\nu_{-} \wedge u^{-},\left(\delta u^{-}\right)^{c}\right\rangle d \sigma-\mu \int_{\partial \Omega}\left\langle\nu_{-} \wedge u^{-},\left(k e_{4} \odot u^{-}\right)^{c}\right\rangle d \sigma \\
+\mu \int_{\partial \Omega}\left\langle\nu_{-} \vee u^{-},\left(d u^{-}\right)^{c}\right\rangle d \sigma+\mu \int_{\partial \Omega}\left\langle\nu_{-} \vee u^{-},\left(k e_{4} \odot u^{-}\right)^{c}\right\rangle d \sigma .
\end{aligned}
$$


Adding together (5.53) and (5.52) then yields, after observing that all boundary integrals cancel,

$$
\begin{aligned}
\int_{\Omega_{+}}\left(\left|d u^{+}\right|^{2}+\left|\delta u^{+}\right|^{2}\right) d x & +\mu \int_{\Omega_{-}}\left(\left|d u^{-}\right|^{2}+\left|\delta u^{-}\right|^{2}\right) d x \\
& =\left(k^{2}\right)^{c}\left(\int_{\Omega_{+}}\left|u^{+}\right|^{2} d x+\mu \int_{\Omega_{-}}\left|u^{-}\right|^{2} d x\right) .
\end{aligned}
$$

Finally, comparing (5.46) with (5.54) gives

$$
\left(\left(k^{2}\right)^{c}-|k|^{2}\right)\left(\int_{\Omega_{+}}\left|u^{+}\right|^{2} d x+\mu \int_{\Omega_{-}}\left|u^{-}\right|^{2} d x\right)=0 .
$$

Therefore, for any $k \in \mathbb{C}$ with $\operatorname{Im} k>0$, the above analysis proves that $u^{+}=0$ in $\Omega_{+}$and $u^{-}=0$ in $\Omega_{-}$. Upon recalling that $u^{ \pm}:=\mathscr{C}_{k} f$ in $\Omega_{ \pm}$, and keeping in mind the jump-formulas for $\mathscr{C}_{k}$ from Theorem 4.5 it follows from what we have just proved that

$$
0=\left.u^{+}\right|_{\partial \Omega}-\left.u^{-}\right|_{\partial \Omega}=-\nu \odot f \quad \text { on } \partial \Omega .
$$

Multiplying the extreme sides of the above equality by $\nu$, we may conclude that $f=0$. Therefore, $\lambda I+\nu \wedge C_{k}$ is one-to-one as an operator from $L_{n o r}^{2, d}\left(\partial \Omega, \mathcal{C}_{4}\right)$ into itself for each real $\lambda \geq \frac{1}{2}$ and each $k \in \mathbb{C}$ with $\operatorname{Im} k>0$. A similar analysis works in the case when $\lambda \leq-\frac{1}{2}$, completing the proof of the lemma.

As mentioned earlier, Lemma 5.7 plays a crucial role in the proof of Theorem5.1, Before turning to the proof of this theorem we single out another useful ingredient, the so-called Analytic Fredholm Alternative (cf., e.g., La93 for a proof).

Theorem 5.8. Let $\mathscr{X}$ be a Banach space and denote by $\mathscr{L}(\mathscr{X})$ the Banach space of all linear and bounded operators from $\mathscr{X}$ into itself (equipped with the operator norm) and by $\mathscr{K}(\mathscr{X})$ the two-sided closed ideal of $\mathscr{L}(\mathscr{X})$ consisting of compact operators on $\mathscr{X}$. Also, let $\mathscr{O}$ be an open subset of $\mathbb{C}$. Next, assume that

$$
A: \mathscr{O} \longrightarrow \mathscr{K}(\mathscr{X})
$$

is an analytic mapping, i.e. $\frac{\partial}{\partial \bar{z}} A(z)=0$ for any $z$ in $\mathscr{O}$, with the property that there exists $z_{0} \in \mathscr{O}$ such that $A\left(z_{0}\right)=0$. Then there exists a subset $E$ of $\mathscr{O}$, which has no accumulation points in $\mathscr{O}$, such that, with $I \in \mathscr{L}(\mathscr{X})$ denoting the identity operator on $\mathscr{X}$,

$$
I+A(z): \mathscr{X} \longrightarrow \mathscr{X}
$$

is invertible for every $z \in \mathscr{O} \backslash E$.

After this lengthy preamble, here is the endgame in the

Proof of Theorem [5.1. Fix $\lambda \in \mathbb{R}$ with $|\lambda| \geq \frac{1}{2}$ along with $k_{0} \in \mathbb{C}, \operatorname{Im} k_{0}>0$, and write

$$
\lambda I+\nu \wedge C_{k}=\lambda I+\nu \wedge C_{k_{0}}+\nu \wedge\left(C_{k}-C_{k_{0}}\right)=\left(\lambda I+\nu \wedge C_{k_{0}}\right) \circ T_{k}
$$

where, for each $k \in \mathbb{C}$, we have set (with the inverse taken in the sense of Lemma 5.7)

$$
T_{k}:=I+\left(\lambda I+\nu \wedge C_{k_{0}}\right)^{-1} \circ\left[\nu \wedge\left(C_{k}-C_{k_{0}}\right)\right] \in \mathscr{L}\left(L_{\text {nor }}^{2, d}\left(\partial \Omega, \mathcal{C}_{4}\right)\right) .
$$


Note that, by virtue of Lemma 5.6, (5.4) and Lemma 4.1, we have that

$$
\begin{gathered}
A(k):=\left(\lambda I+\nu \wedge C_{k_{0}}\right)^{-1} \circ\left[\nu \wedge\left(C_{k}-C_{k_{0}}\right)\right] \in \mathscr{K}\left(L_{\text {nor }}^{2, d}\left(\partial \Omega, \mathcal{C l}_{4}\right)\right) \\
\text { for every } k \in \mathbb{C} .
\end{gathered}
$$

Furthermore, by (4.8), (4.1) and (4.14), the mapping

$$
\mathbb{C} \ni k \mapsto A(k) \in \mathscr{K}\left(L_{\text {nor }}^{2, d}\left(\partial \Omega, \mathcal{C l}_{4}\right)\right)
$$

is analytic. Furthermore, $A\left(k_{0}\right)=0$. Thus, Theorem 5.8 applies and gives that there exists an exceptional subset $E$ of $\mathbb{C}$, which has no finite accumulation points, such that $T_{k}$ is invertible on $L_{\text {nor }}^{2, d}\left(\partial \Omega, \mathcal{C l}_{4}\right)$ for any $k \in \mathbb{C} \backslash E$. From (5.59), we know that $\lambda I+\nu \wedge C_{k}$ is invertible on $L_{\text {nor }}^{2, d}\left(\partial \Omega, \mathcal{C}_{4}\right)$ if and only if $T_{k}$ is invertible on this space. Therefore, the operator $\lambda I+\nu \wedge C_{k}$ is invertible on $L_{n o r}^{2, d}\left(\partial \Omega, C_{4}\right)$ for any $k$ in $\mathbb{C} \backslash E$. Given that, by Lemma 5.7 the operator $\lambda I+\nu \wedge C_{k}$ is also invertible on $L_{\text {nor }}^{2, d}\left(\partial \Omega, C_{4}\right)$ whenever $k \in \mathbb{C}_{+}$, it follows that actually $\lambda I+\nu \wedge C_{k}$ is invertible on $L_{\text {nor }}^{2, d}\left(\partial \Omega, \mathcal{C l}_{4}\right)$ whenever $k \in \mathbb{C} \backslash(E \cap \mathbb{R})$ has $\operatorname{Im} k \geq 0$. Since $E$ has no finite accumulation points, $E \cap \mathbb{R}$ is a countable set of real numbers which eventually accumulates only at $\pm \infty$. Hence, $E \cap \mathbb{R}$ is an at most countable set, say $E \cap \mathbb{R}=:\left\{k_{j}\right\}_{j}$, where all $k_{j}$ 's are real numbers. Furthermore, without any loss of generality, we may assume that $0 \in\left\{k_{j}\right\}_{j}$. In summary, so far we have proved that for any $\lambda$ real with $|\lambda| \geq \frac{1}{2}$, there exists an at most countable set $\left\{k_{j}\right\}_{j}$ of real numbers, which contains 0 and possibly accumulates only at $\pm \infty$, such that the operator $\lambda I+\nu \wedge C_{k}$ is an isomorphism of $L_{\text {nor }}^{2, d}\left(\partial \Omega, C_{4}\right)$ for any $k \in \mathbb{C} \backslash\left\{k_{j}\right\}_{j}$ with $\operatorname{Im} k \geq 0$.

Having established this, we now focus on showing that if $\varepsilon=\varepsilon(\Omega)>0$ is as in (5.1) and the set $\left\{k_{j}\right\}_{j}$ is as above, then the operator $\lambda I+\nu \wedge C_{k}$ is an isomorphism of $L_{n o r}^{p, d}\left(\partial \Omega, C_{4}\right)$ for any $k \in \mathbb{C} \backslash\left\{k_{j}\right\}_{j}$ with $\operatorname{Im} k \geq 0$ whenever $p \in(1,2+\varepsilon)$. To prove this recall from (5.1) that one can choose $\varepsilon=\varepsilon(\Omega)>0$ such that the operator $\lambda I+\nu \wedge C_{k}$ is Fredholm with index zero on $L_{\text {nor }}^{p, d}\left(\partial \Omega, \mathcal{C l}_{4}\right)$ whenever $k \in \mathbb{C}$ has $\operatorname{Im} k \geq 0, \lambda \in \mathbb{R}$ satisfies $|\lambda| \geq \frac{1}{2}$ and $1<p<2+\varepsilon$. Hence, the desired conclusion follows as soon as we show that the operator $\lambda I+\nu \wedge C_{k}$ acting on the space $L_{\text {nor }}^{p, d}\left(\partial \Omega, \mathcal{C l}_{4}\right)$ is either one-to-one or has dense range whenever $1<p<2+\varepsilon$ and $k \in \mathbb{C} \backslash\left\{k_{j}\right\}_{j}$ has $\operatorname{Im} k \geq 0$. However, given that $\partial \Omega$ is compact and, hence,

$$
\begin{gathered}
L_{\text {nor }}^{p, d}\left(\partial \Omega, \mathcal{C l}_{4}\right) \hookrightarrow L_{\text {nor }}^{2, d}\left(\partial \Omega, \mathcal{C l}_{4}\right) \quad \text { if } p \geq 2 \text { and } \\
L_{\text {nor }}^{2, d}\left(\partial \Omega, \mathcal{C l}_{4}\right) \hookrightarrow L_{\text {nor }}^{p, d}\left(\partial \Omega, \mathcal{C l}_{4}\right) \text { if } p \leq 2,
\end{gathered}
$$

with both inclusions with dense ranges, it follows from Lemma 5.7 that $\lambda I+\nu \wedge C_{k}$ is injective when acting on the space $L_{\text {nor }}^{p, d}\left(\partial \Omega, \mathcal{C l}_{4}\right)$ for $p \in[2, \infty)$, and has dense range when acting on the space $L_{\text {nor }}^{p, d}\left(\partial \Omega, C \ell_{4}\right)$ for $p \in(1,2]$. This finishes the proof of Theorem 5.1

We conclude this section by presenting a version of Theorem 5.1 which deals with the invertibility of the operator $\lambda I+\nu \vee C_{k}$.

Theorem 5.9. Let $\Omega \subset \mathbb{R}^{3}$ be an arbitrary Lipschitz domain with compact boundary. Then there exists $\varepsilon=\varepsilon(\Omega)>0$ with the following significance. For every $\lambda \in \mathbb{R}$ with $|\lambda| \geq \frac{1}{2}$ there exists an at most countable set of real numbers $\left\{k_{j}\right\}_{j}$ containing 0 and possibly accumulating only at $\pm \infty$, such that whenever $1<p<2+\varepsilon$ and $k \in \mathbb{C} \backslash\left\{k_{j}\right\}_{j}$ with $\operatorname{Im} k \geq 0$, the operator $\lambda I+\nu \vee C_{k}$ is an isomorphism of $L_{\text {tan }}^{p, \delta}\left(\partial \Omega, \mathcal{C}_{4}\right)$. 
Proof. The proof is similar to the one of Theorem 5.1

\section{Half-Dirichlet problems for Perturbed Dirac operators}

In this section, we consider the half-Dirichlet problems for perturbed Dirac operators in Lipschitz domains. To set the stage, let $\Omega \subset \mathbb{R}^{3}$ be a bounded Lipschitz domain and recall (1.1). For $p \in(1, \infty)$ and $k \in \mathbb{C}$ with $\operatorname{Im} k \geq 0$ fixed, introduce the interior Hardy space

$$
\begin{array}{r}
\mathscr{H}_{k}^{p}\left(\Omega_{+}\right):=\left\{u \in C^{1}\left(\Omega_{+}, \mathcal{C l}_{4}\right): \mathbb{D}_{k} u=0 \text { in } \Omega_{+},\right. \\
\text {and } \left.N(u), N(d u), N(\delta u) \in L^{p}(\partial \Omega)\right\},
\end{array}
$$

as well as the exterior Hardy space

$$
\begin{aligned}
\mathscr{H}_{k}^{p}\left(\Omega_{-}\right):= & \left\{u \in C^{1}\left(\Omega_{-}, \mathcal{C}_{4}\right): \mathbb{D}_{k} u=0 \text { in } \Omega_{-},\right. \\
& \left.N(u), N(d u), N(\delta u) \in L^{p}(\partial \Omega) \text { and } u \text { decays at infinity }\right\} .
\end{aligned}
$$

Lemma 6.1. Let $\Omega \subset \mathbb{R}^{3}$ be a bounded Lipschitz domain and fix $k \in \mathbb{C} \backslash\{0\}$ with $\operatorname{Im} k \geq 0$ and $p \in(1, \infty)$. Then

$$
u^{ \pm} \in \mathscr{H}_{k}^{p}\left(\Omega_{ \pm}\right) \Longrightarrow\left\{\begin{array}{l}
\left.\nu \wedge u^{ \pm}\right|_{\partial \Omega} \in L_{\text {nor }}^{p, d}\left(\partial \Omega, \mathcal{C l}_{4}\right) \text { and } \\
\left.\nu \vee u^{ \pm}\right|_{\partial \Omega} \in L_{\text {tan }}^{p, \delta}\left(\partial \Omega, \mathcal{C l}_{4}\right)
\end{array}\right.
$$

Proof. This is a direct consequence of (6.1)-(6.2) and Lemma 3.2

In conjunction with the Hardy spaces (6.1)-(6.2), and while continuing to assume that $\Omega \subset \mathbb{R}^{3}$ is a bounded Lipschitz domain, $p \in(1, \infty)$ and $k \in \mathbb{C}$ has $\operatorname{Im} k \geq 0$, consider next the following four half-Dirichlet boundary value problems:

$$
\left\{\begin{array} { l } 
{ u ^ { + } \in \mathscr { H } _ { k } ^ { p } ( \Omega _ { + } ) , } \\
{ \nu \wedge u ^ { + } | _ { \partial \Omega } = f \in L _ { \text { nor } } ^ { p , d } ( \partial \Omega , C _ { 4 } ) , }
\end{array} \quad \left\{\begin{array}{l}
u^{-} \in \mathscr{H}_{k}^{p}\left(\Omega_{-}\right), \\
\left.\nu \wedge u^{-}\right|_{\partial \Omega}=f \in L_{\text {nor }}^{p, d}\left(\partial \Omega, C_{4}\right)
\end{array}\right.\right.
$$

and

$$
\left\{\begin{array} { l } 
{ u ^ { + } \in \mathscr { H } _ { k } ^ { p } ( \Omega _ { + } ) , } \\
{ \nu \vee u ^ { + } | _ { \partial \Omega } = g \in L _ { \text { tan } } ^ { p , \delta } ( \partial \Omega , \mathcal { C l } _ { 4 } ) , }
\end{array} \quad \left\{\begin{array}{l}
u^{-} \in \mathscr{H}_{k}^{p}\left(\Omega_{-}\right), \\
\left.\nu \vee u^{-}\right|_{\partial \Omega}=g \in L_{\text {tan }}^{p, \delta}\left(\partial \Omega, \mathcal{C l}_{4}\right) .
\end{array}\right.\right.
$$

The terminology "half-Dirichlet" is justified by the fact that the boundary data $\left.\nu \wedge u^{ \pm}\right|_{\partial \Omega},\left.\nu \vee u^{ \pm}\right|_{\partial \Omega}$ satisfy $\nu \vee\left(\left.\nu \wedge u^{ \pm}\right|_{\partial \Omega}\right)+\nu \wedge\left(\left.\nu \vee u^{ \pm}\right|_{\partial \Omega}\right)=\left.u^{ \pm}\right|_{\partial \Omega}$, the latter corresponding to what would ordinarily constitute the Dirichlet data for a second order elliptic differential operator. We wish to stress that this is natural, since $u^{ \pm}$ are null-solutions of a perturbed Dirac operator which is, of course, a first-order differential operator.

Theorem 6.2. Assume that $\Omega \subset \mathbb{R}^{3}$ is a bounded Lipschitz domain. Then there exists a number $\varepsilon=\varepsilon(\Omega)>0$ and an at most countable set of real numbers $\left\{k_{j}\right\}_{j}$ such that, whenever $1<p<2+\varepsilon$ and $k \in \mathbb{C} \backslash\left\{k_{j}\right\}_{j}$ has $\operatorname{Im} k \geq 0$, the four boundary value problems listed in (6.4)-(6.5) are all well-posed.

Proof. Let $\varepsilon>0$ and $\left\{k_{j}\right\}_{j}$ be such that the conclusions in Theorems 5.15 .9 hold, and suppose that $k, p$ are as in the statement of the theorem. Then it follows from Theorem 5.1, Theorem 4.3, Theorem 4.5 and Proposition 4.6 that

$$
u^{ \pm}:=\mathscr{C}_{k}\left[\left( \pm \frac{1}{2} I+\nu \wedge C_{k}\right)^{-1} f\right] \quad \text { in } \Omega_{ \pm}
$$


are solutions to the two boundary value problems listed in (6.4). Note that, by (6.6) and Theorem 4.3, these functions also satisfy

$$
\left\|N\left(u^{ \pm}\right)\right\|_{L^{p}(\partial \Omega)}+\left\|N\left(d u^{ \pm}\right)\right\|_{L^{p}(\partial \Omega)}+\left\|N\left(\delta u^{ \pm}\right)\right\|_{L^{p}(\partial \Omega)} \leq C\|f\|_{L_{n o r}^{p, d}\left(\partial \Omega, C_{4}\right)},
$$

for some $C=C(\Omega, k, p)>0$ independent of $f$. As far as uniqueness is concerned, if $u^{+}$and $u^{-}$solve the homogeneous versions of the problems listed in (6.4), start with the Cauchy reproducing formulas (4.40), (4.42) written for $u^{ \pm}$, then take nontangential boundary traces and, finally, apply $\nu \wedge$ to both sides of the resulting formulas in order to obtain

$$
\left(\mp \frac{1}{2} I+\nu \vee C_{k}\right)\left(\left.\nu \vee u^{ \pm}\right|_{\partial \Omega}\right)=0 \quad \text { on } \partial \Omega .
$$

Based on this and Theorem 5.1 we may then conclude that $\nu \vee u^{ \pm}=0$ on $\partial \Omega$. With this in hand and upon recalling that we are assuming that the homogeneous versions of the boundary conditions in (6.4) hold, it follows that $u^{ \pm}=0$ on $\partial \Omega$. Hence, further, $u^{ \pm}=0$ in $\Omega_{ \pm}$thanks to the Cauchy reproducing formulas (4.40), (4.42). This completes the proof of uniqueness, and finishes the proof of the wellposedness of the two problems in (6.4).

Similarly, by Theorem 5.9, Theorem 4.3, Theorem 4.5, Proposition 4.6, and reasoning for uniqueness as above, we deduce that

$$
u^{ \pm}:=\mathscr{C}_{k}\left[\left( \pm \frac{1}{2} I+\nu \vee C_{k}\right)^{-1} g\right] \quad \text { in } \Omega_{ \pm}
$$

are the unique solutions to the two boundary value problem listed in (6.5) and satisfy estimates analogous to (6.7).

Corollary 6.3. In the context of Theorem 6.2 , if $u^{ \pm} \in \mathscr{H}_{k}^{p}\left(\Omega_{ \pm}\right)$, then

$$
u^{ \pm}=\mathscr{C}_{k}\left[\left(\mp \frac{1}{2} I+\nu \vee C_{k}\right)^{-1}\left(\left.\nu \vee u^{ \pm}\right|_{\partial \Omega}\right)\right] \quad \text { in } \Omega_{ \pm}
$$

and

$$
u^{ \pm}=\mathscr{C}_{k}\left[\left( \pm \frac{1}{2} I+\nu \wedge C_{k}\right)^{-1}\left(\left.\nu \wedge u^{ \pm}\right|_{\partial \Omega}\right)\right] \quad \text { in } \Omega_{ \pm} .
$$

Proof. For any $u^{ \pm} \in \mathscr{H}_{k}^{p}\left(\Omega_{ \pm}\right)$, Lemma 6.1 ensures that $\left.\nu \wedge u^{ \pm}\right|_{\partial \Omega} \in L_{\text {nor }}^{p, d}\left(\partial \Omega, \mathcal{C l}_{4}\right)$ and that $\left.\nu \vee u^{ \pm}\right|_{\partial \Omega} \in L_{\text {tan }}^{p, \delta}\left(\partial \Omega, \mathcal{C l}_{4}\right)$. Consequently, (6.10)-(6.11) follow from this observation, Theorem 6.2, and the integral representation formulas (6.6)-(6.9).

Next we would like to consider the invertibility of the Cauchy type operators $\nu \wedge C_{k}$ and $\nu \vee C_{k}$, which is going to be of importance later on, in the proof of the main result of the paper, Theorem 7.1 (when moving from (7.12) to (7.13), to be more precise). The context is that of a bounded Lipschitz domain $\Omega \subset \mathbb{R}^{3}$ and $\varepsilon>0$ and $\left\{k_{j}\right\}_{j}$ are such that the conclusions in Theorems 5.1]5.9 are valid. Also, fix $p \in(1,2+\varepsilon)$ along with $k \in \mathbb{C} \backslash\left\{k_{j}\right\}_{j}$ with $\operatorname{Im} k \geq 0$. We begin by introducing the tangential-to-normal operators $\mathrm{TN}_{k}^{ \pm}$mapping functions from $L_{\text {tan }}^{p, \delta}\left(\partial \Omega, \mathcal{C l}_{4}\right)$ into functions from $L_{n o r}^{p, d}\left(\partial \Omega, \mathcal{C l}_{4}\right)$ according to

$$
\mathrm{TN}_{k}^{ \pm}(g):=\left.\nu \wedge\left[\mathscr{C}_{k}\left(\left(\mp \frac{1}{2} I+\nu \vee C_{k}\right)^{-1} g\right)\right]\right|_{\partial \Omega_{ \pm}}, \quad \forall g \in L_{t a n}^{p, \delta}\left(\partial \Omega, \mathcal{C l}_{4}\right)
$$

In the aforementioned context, $\mathrm{TN}_{k}^{ \pm}$is a well-defined, linear and bounded operator since $d_{\partial}\left(\mathrm{TN}_{k}^{ \pm}(g)\right)=\left.\nu \wedge\left(d \mathscr{S}_{k}\left(\delta_{\partial} \widetilde{g}\right)-k e_{4} \odot d \mathscr{S}_{k} \widetilde{g}\right)\right|_{\partial \Omega}$, where $\widetilde{g}:=\left(\mp \frac{1}{2} I+\nu \vee C_{k}\right)^{-1} g$. The name "tangential-to-normal" is justified by the observation that

$$
\mathrm{TN}_{k}^{ \pm}\left(\left.\nu \vee u^{ \pm}\right|_{\partial \Omega}\right)=\left.\nu \wedge u^{ \pm}\right|_{\partial \Omega}, \quad \forall u^{ \pm} \in \mathscr{H}_{k}^{p}\left(\Omega_{ \pm}\right)
$$


as can be seen from (6.10) and (6.12). In the same setting, we also define the normal-to-tangential operators $\mathrm{NT}_{k}^{ \pm}$which map $L_{n o r}^{p, d}\left(\partial \Omega, \mathcal{C l}_{4}\right)$ into $L_{t a n}^{p, \delta}\left(\partial \Omega, \mathcal{C l}_{4}\right)$ according to

$$
\mathrm{NT}_{k}^{ \pm}(f):=\left.\nu \vee\left[\mathscr{C}_{k}\left(\left( \pm \frac{1}{2} I+\nu \wedge C_{k}\right)^{-1} f\right)\right]\right|_{\partial \Omega_{ \pm}}, \quad \forall f \in L_{n o r}^{p, d}\left(\partial \Omega, \mathcal{C}_{4}\right) .
$$

Once again, the terminology "normal-to-tangential" is natural since, by (6.11) and (6.13),

$$
\mathrm{NT}_{k}^{ \pm}\left(\left.\nu \wedge u^{ \pm}\right|_{\partial \Omega}\right)=\left.\nu \vee u^{ \pm}\right|_{\partial \Omega}, \quad \forall u^{ \pm} \in \mathscr{H}_{k}^{p}\left(\Omega_{ \pm}\right)
$$

Alternative expressions for the operators $\mathrm{TN}_{k}^{ \pm}$and $\mathrm{NT}_{k}^{ \pm}$are developed in the following lemma.

Lemma 6.4. In the same setting as above, the operators $\mathrm{TN}_{k}^{ \pm}$and $\mathrm{NT}_{k}^{ \pm}$satisfy

$$
\begin{gathered}
\mathrm{TN}_{k}^{ \pm}=\left(\nu \wedge C_{k}\right) \circ\left(\mp \frac{1}{2} I+\nu \vee C_{k}\right)^{-1} \quad \text { and } \\
\mathrm{NT}_{k}^{ \pm}=\left(\nu \vee C_{k}\right) \circ\left( \pm \frac{1}{2} I+\nu \wedge C_{k}\right)^{-1}
\end{gathered}
$$

Proof. If $g \in L_{t a n}^{p, \delta}\left(\partial \Omega, C_{4}\right)$ and if we set $h^{ \pm}:=\left(\mp \frac{1}{2} I+\nu \vee C_{k}\right)^{-1} g$, it follows that $h^{ \pm} \in L_{\text {tan }}^{p, \delta}\left(\partial \Omega, C_{4}\right)$. In particular, $\nu \vee h^{ \pm}=0$. Using this and (4.36) we then obtain

$$
\mathrm{TN}_{k}^{ \pm}(g)=\left.\nu \wedge\left(\mathscr{C}_{k} h^{ \pm}\right)\right|_{\partial \Omega_{ \pm}}=\nu \wedge C_{k} h^{ \pm}=\left(\nu \wedge C_{k}\right)\left(\mp \frac{1}{2} I+\nu \vee C_{k}\right)^{-1} g .
$$

Therefore, $\mathrm{TN}_{k}^{ \pm}=\left(\nu \wedge C_{k}\right) \circ\left(\mp \frac{1}{2} I+\nu \vee C_{k}\right)^{-1}$, proving the first formula in (6.16). The second formula in (6.16) is proved in a similar way, and this finishes the proof of the lemma.

Theorem 6.5. Let $\Omega$ be a bounded Lipschitz domain in $\mathbb{R}^{3}$. Then there exist $\varepsilon=\varepsilon(\Omega)>0$ and an at most countable set of real numbers $\left\{k_{j}\right\}_{j}$ such that if $1<p<2+\varepsilon$ and $k \in \mathbb{C} \backslash\left\{k_{j}\right\}_{j}$ has $\operatorname{Im} k \geq 0$, the operators $\mathrm{TN}_{k}^{ \pm}$and $\mathrm{NT}_{k}^{ \pm}$are linear, bounded, and satisfy

$$
\begin{gathered}
\mathrm{TN}_{k}^{ \pm} \circ \mathrm{NT}_{k}^{ \pm}=I \text { on } L_{n o r}^{p, d}\left(\partial \Omega, \mathcal{C l}_{4}\right) \quad \text { and } \\
\mathrm{NT}_{k}^{ \pm} \circ \mathrm{TN}_{k}^{ \pm}=I \text { on } L_{\text {tan }}^{p, \delta}\left(\partial \Omega, \mathcal{C l}_{4}\right),
\end{gathered}
$$

where I stands for the identity operator. In particular, under the same background assumptions, the operators

$$
\begin{aligned}
\mathrm{TN}_{k}^{ \pm}: L_{\text {tan }}^{p, \delta}\left(\partial \Omega, \mathcal{C} \ell_{4}\right) \longrightarrow L_{\text {nor }}^{p, d}\left(\partial \Omega, \mathcal{C l}_{4}\right), \\
\mathrm{NT}_{k}^{ \pm}: L_{\text {nor }}^{p, d}\left(\partial \Omega, \mathcal{C l}_{4}\right) \longrightarrow L_{\text {tan }}^{p, \delta}\left(\partial \Omega, \mathcal{C l}_{4}\right)
\end{aligned}
$$

are isomorphisms.

Proof. Let $\varepsilon>0$ and $\left\{k_{j}\right\}_{j}$ be such that the conclusions in Theorems 5.1 5.9 hold. The fact that, in the setting described in the current theorem, the operators $\mathrm{TN}_{k}^{ \pm}, \mathrm{NT}_{k}^{ \pm}$are linear are bounded follows from (6.16), Theorems 5.1 5.9 and the Calderón-Zygmund theory. Moreover, the two operator identities in (6.18) are consequences of (6.13), (6.15) and Theorem 6.2. Of course, the fact that (6.19)(6.20) are isomorphisms is a direct corollary of (6.18).

Here are the invertibility results for the operators $\nu \wedge C_{k}, \nu \vee C_{k}$ mentioned earlier. 
Corollary 6.6. Let $\Omega$ be a bounded Lipschitz domain in $\mathbb{R}^{3}$. Then there exist $\varepsilon>0$ and an at most countable set of real numbers $\left\{k_{j}\right\}_{j}$ such that whenever $1<p<2+\varepsilon$ and $k \in \mathbb{C} \backslash\left\{k_{j}\right\}_{j}$ has $\operatorname{Im} k \geq 0$, the operators

$$
\begin{aligned}
& \nu \wedge C_{k}: L_{\text {tan }}^{p, \delta}\left(\partial \Omega, \mathcal{C}_{4}\right) \longrightarrow L_{\text {nor }}^{p, d}\left(\partial \Omega, \mathcal{C l}_{4}\right), \\
& \nu \vee C_{k}: L_{\text {nor }}^{p, d}\left(\partial \Omega, \mathcal{C l}_{4}\right) \longrightarrow L_{\text {tan }}^{p, \delta}\left(\partial \Omega, \mathcal{C l}_{4}\right)
\end{aligned}
$$

are isomorphisms.

Proof. Assume that $\varepsilon>0$ and $\left\{k_{j}\right\}_{j}$ are such that the conclusions in Theorems 5.1 . 5.9 are valid, and suppose that $p$ and $k$ are as in the statement of the corollary. Then, due to (6.16), we may write

$$
\nu \wedge C_{k}=\mathrm{TN}_{k}^{+} \circ\left(-\frac{1}{2} I+\nu \vee C_{k}\right) .
$$

Since by Theorem 6.5 and Theorem 5.9 the operators $\mathrm{TN}_{k}^{+}$and $-\frac{1}{2} I+\nu \vee C_{k}$ are both isomorphisms, it follows that $\nu \wedge C_{k}$ is an isomorphism as well, in the context of (6.21). A similar argument works in the case of $\nu \vee C_{k}$, in the context of (6.22).

\section{The transmission Problem for PERTURbed Dirac operators}

In this section we prove the main result of this paper, namely the well-posedness of the transmission boundary value problem for perturbed Dirac operators in threedimensional Lipschitz domains. To set the stage, consider a bounded Lipschitz domain $\Omega$ in $\mathbb{R}^{3}$ and assume that a transmission parameter $\mu \in(0,1)$ along with a wave number $k \in \mathbb{C} \backslash\{0\}$ with $\operatorname{Im} k \geq 0$ have been fixed. Also, assume that $p \in(1, \infty)$ and that $u^{ \pm} \in C^{1}\left(\Omega_{ \pm}, \mathcal{C} \ell_{4}\right)$. Then the transmission boundary value problem in question reads

$$
\left\{\begin{array}{l}
\mathbb{D}_{k} u^{ \pm}=0 \text { in } \Omega_{ \pm} \\
\left.\nu \vee u^{+}\right|_{\partial \Omega}-\left.\nu \vee u^{-}\right|_{\partial \Omega}=g \in L^{p}\left(\partial \Omega, \mathcal{C}_{4}\right), \\
\left.\nu \wedge u^{+}\right|_{\partial \Omega}-\left.\mu \nu \wedge u^{-}\right|_{\partial \Omega}=f \in L^{p}\left(\partial \Omega, C_{4}\right), \\
u^{-} \text {decays at infinity, } \\
N\left(u^{ \pm}\right), N\left(d u^{ \pm}\right), N\left(\delta u^{ \pm}\right) \in L^{p}(\partial \Omega) .
\end{array}\right.
$$

The theorem below identifies the functional analytical setting in which (7.1) is wellposed. In particular, it highlights the basic role which the spaces $L_{t a n}^{p, \delta}\left(\partial \Omega, \mathcal{C l}_{4}\right)$ and $L_{\text {nor }}^{p, d}\left(\partial \Omega, \mathcal{C l}_{4}\right)$ play in this regard.

Theorem 7.1. Retain the above setup. Then there exist $\varepsilon>0$ and an at most countable set of real numbers $\left\{k_{j}\right\}_{j}$ which depend exclusively on the boundary $\partial \Omega$ and which have the following significance. Whenever $p \in(1,2+\varepsilon), k \in \mathbb{C} \backslash\left\{k_{j}\right\}_{j}$ has $\operatorname{Im} k \geq 0$, and $\mu \in(0,1)$, the Dirac transmission problem (7.1) has a solution if and only if

$$
g \in L_{t a n}^{p, \delta}\left(\partial \Omega, \mathcal{C}_{4}\right) \text { and } f \in L_{\text {nor }}^{p, d}\left(\partial \Omega, \mathcal{C l}_{4}\right) .
$$


Furthermore, the solution $\left(u^{+}, u^{-}\right)$is unique and satisfies the estimate

$$
\begin{aligned}
\left\|N\left(u^{ \pm}\right)\right\|_{L^{p}(\partial \Omega)}+ & \left\|N\left(d u^{ \pm}\right)\right\|_{L^{p}(\partial \Omega)}+\left\|N\left(\delta u^{ \pm}\right)\right\|_{L^{p}(\partial \Omega)} \\
& \leq C\left(\|g\|_{L_{t a n}^{p, \delta}\left(\partial \Omega, \ell_{4}\right)}+\|f\|_{L_{\text {nor }}^{p, d}\left(\partial \Omega, \ell_{4}\right)}\right),
\end{aligned}
$$

where $C=C(\partial \Omega, k, p)>0$ is independent of $f$ and $g$.

Proof. The necessity of (7.2) follows from the format of the boundary conditions in (7.1) and Lemma 6.1. Parenthetically, we wish to point out that this portion of analysis works without any supplementary restrictions on $p \in(1, \infty)$ and $k \in \mathbb{C}$. For the sufficiency part, assume that $\varepsilon>0$ and the at most countable set of real numbers $\left\{k_{j}\right\}_{j}$ are such that the results from Sections 5 [6 apply. Next, fix $p \in(1,2+\varepsilon), k \in \mathbb{C} \backslash\left\{k_{j}\right\}_{j}$ with $\operatorname{Im} k \geq 0$, and pick two arbitrary functions, $f \in L_{\text {nor }}^{p, d}\left(\partial \Omega, \mathcal{C l}_{4}\right)$ and $g \in L_{\text {tan }}^{p, \delta}\left(\partial \Omega, \mathcal{C l}_{4}\right)$. In order to solve the above transmission boundary value problem in this setting, we shall make use of two related, auxiliary boundary value problems, which we now proceed to describe. First, consider the half-Dirichlet boundary value problem

$$
\left\{\begin{array}{l}
\mathbb{D}_{k} u=0 \text { in } \Omega_{+}, \\
\left.\nu \vee u\right|_{\partial \Omega}=g \\
N(u), N(d u), N(\delta u) \in L^{p}(\partial \Omega) .
\end{array}\right.
$$

Granted the current assumptions, Theorem 6.5 then gives that this problem is well-posed. Second, we set

$$
\widetilde{f}:=f-\left.\nu \wedge u\right|_{\partial \Omega} \in L_{\text {nor }}^{p, d}\left(\partial \Omega, \mathcal{C l}_{4}\right)
$$

(where the membership is a consequence of background assumptions, (7.4) and Lemma 6.1), and consider what can be termed as a "reduced" transmission boundary value problem (in the sense that one of the boundary data is zero), i.e.,

$$
\left\{\begin{array}{l}
\mathbb{D}_{k} v^{ \pm}=0 \text { in } \Omega_{ \pm}, \\
\left.\nu \vee v^{+}\right|_{\partial \Omega}-\left.\nu \vee v^{-}\right|_{\partial \Omega}=0, \\
\left.\nu \wedge v^{+}\right|_{\partial \Omega}-\left.\mu \nu \wedge v^{-}\right|_{\partial \Omega}=\widetilde{f}, \\
v^{-} \text {decays at infinity, } \\
N\left(v^{ \pm}\right), N\left(d v^{ \pm}\right), N\left(\delta v^{ \pm}\right) \in L^{p}(\partial \Omega) .
\end{array}\right.
$$

Observe that once the problem (17.6) has been solved (with naturally accompanying estimates), then a solution for the original transmission boundary value problem (17.11) may be found by setting

$$
u^{+}:=u+v^{+} \text {in } \Omega_{+} \quad \text { and } \quad u^{-}:=v^{-} \text {in } \Omega_{-},
$$

where $u$ and $v^{ \pm}$solve (7.4) and (7.6), respectively. Furthermore, by (6.7) used for $u$ plus the presumed estimates for $v^{ \pm}$, the functions $u^{ \pm}$from (7.7) also satisfy (7.3).

As such, matters are reduced to solving (17.6). To this end, let $h \in L_{\text {nor }}^{p, d}\left(\partial \Omega, \mathcal{C}_{4}\right)$, to be specified later, and define $v^{ \pm}:=\mathscr{C}_{k} h$ in $\Omega_{ \pm}$. Clearly, $\mathbb{D}_{k} v^{ \pm}=0$ in $\Omega_{ \pm}$, and Proposition 4.6 guarantees that $v^{-}$decays at infinity in the desired fashion. Moreover, the validity of the last condition in (7.6) is ensured by Theorem 4.3. 
whereas the fact that the first boundary condition in (7.6) holds is seen from (4.36). Next, making use of (4.35), it follows that the second boundary condition in (7.6) is equivalent to having

$$
\frac{1}{2}(\mu+1) h+(1-\mu) \nu \wedge C_{k} h=\tilde{f}
$$

To proceed, abbreviate $\lambda:=\frac{1}{2} \frac{1+\mu}{1-\mu}$ so that $\lambda \in \mathbb{R}$ and $\frac{1}{2}<\lambda<\infty$. Dividing both sides of the above equality by $1-\mu$ then allows us to recast this boundary integral equation in the form

$$
\left(\lambda I+\nu \wedge C_{k}\right) h=\frac{1}{1-\mu} \tilde{f} \in L_{n o r}^{p, d}\left(\partial \Omega, \mathcal{C l}_{4}\right) .
$$

By Theorem 5.1, the function $h:=(1-\mu)^{-1}\left(\lambda I+\nu \wedge C_{k}\right)^{-1} \tilde{f} \in L_{\text {nor }}^{p, d}\left(\partial \Omega, \mathcal{C l}_{4}\right)$ solves this equation. This proves that (7.1) is solvable under the specified hypotheses, and that the solution satisfies natural estimates.

As regards the uniqueness part for the problem (7.1), by linearity this comes down to proving that if $\left(w^{+}, w^{-}\right)$solve the homogeneous problem

$$
\left\{\begin{array}{l}
w^{+} \in \mathscr{H}_{k}^{p}\left(\Omega_{+}\right) \text {and } w^{-} \in \mathscr{H}_{k}^{p}\left(\Omega_{-}\right), \\
\left.\nu \vee w^{+}\right|_{\partial \Omega}=\left.\nu \vee w^{-}\right|_{\partial \Omega}, \\
\left.\nu \wedge w^{+}\right|_{\partial \Omega}=\left.\mu \nu \wedge w^{-}\right|_{\partial \Omega},
\end{array}\right.
$$

then necessarily $w^{ \pm}=0$ in $\Omega_{ \pm}$. Let us abbreviate $h:=\left.\nu \vee w^{+}\right|_{\partial \Omega}=\left.\nu \vee w^{-}\right|_{\partial \Omega}$. Then, since $h \in L_{\text {tan }}^{p, \delta}\left(\partial \Omega, \mathcal{C l}_{4}\right)$, by Corollary 6.3. we have

$$
w^{+}=\mathscr{C}_{k}\left[\left(-\frac{1}{2} I+\nu \vee C_{k}\right)^{-1} h\right], \quad w^{-}=\mathscr{C}_{k}\left[\left(\frac{1}{2} I+\nu \vee C_{k}\right)^{-1} h\right] .
$$

From (4.35) and the fact that $\left( \pm \frac{1}{2} I+\nu \vee C_{k}\right)^{-1} h \in L_{\text {tan }}^{p, \delta}\left(\partial \Omega, \mathcal{C l}_{4}\right)$, it follows that the second boundary condition in (7.10) can be re-expressed in the form

$$
\nu \wedge C_{k}\left(-\frac{1}{2} I+\nu \vee C_{k}\right)^{-1} h=\mu \nu \wedge C_{k}\left(-\frac{1}{2} I+\nu \vee C_{k}\right)^{-1} h .
$$

By Corollary 6.6, this further entails

$$
\left(-\frac{1}{2} I+\nu \vee C_{k}\right)^{-1} h=\mu\left(\frac{1}{2} I+\nu \vee C_{k}\right)^{-1} h .
$$

Based on (7.13) and the usual resolvent formula we may then write

$$
\begin{aligned}
\left(\frac{1}{2} I+\nu \vee C_{k}\right)^{-1}\left[\left(\frac{1}{2} I\right.\right. & \left.+\nu \vee C_{k}\right) \\
& \left.-\mu\left(-\frac{1}{2} I+\nu \vee C_{k}\right)\right]\left(-\frac{1}{2} I+\nu \vee C_{k}\right)^{-1} h=0
\end{aligned}
$$

or, after some simple algebra,

$$
(1-\mu)\left(\frac{1}{2} I+\nu \vee C_{k}\right)^{-1}\left(\lambda I+\nu \vee C_{k}\right)\left(-\frac{1}{2} I+\nu \vee C_{k}\right)^{-1} h=0,
$$

where $\lambda:=\frac{1}{2}\left(\frac{1+\mu}{1-\mu}\right) \in\left(\frac{1}{2}, \infty\right)$. By Theorem [5.9] the operators $\left(\frac{1}{2} I+\nu \vee C_{k}\right)^{-1}$, $\lambda I+\nu \vee C_{k}$ and $\left(-\frac{1}{2} I+\nu \vee C_{k}\right)^{-1}$ are all isomorphisms. Hence $h=0$, which, by virtue of (7.11), forces $w^{ \pm}=0$ in $\Omega_{ \pm}$, as desired. This finishes the proof of the uniqueness, hence concluding the proof of the well-posedness of the transmission boundary value problem (7.1). 
In closing, we desire to rewrite the boundary conditions in (7.1) in an alternative, yet equivalent way, which de-emphasizes the use of the exterior and interior products. As a corollary, this is going to show that Theorem 1.1 holds as stated. Concretely, suppose $u^{ \pm}$solve (7.1) for some given $g \in L_{t a n}^{p, \delta}\left(\partial \Omega, C_{4}\right)$ and $f \in L_{\text {nor }}^{p, d}\left(\partial \Omega, C_{4}\right)$. Then

$$
\begin{aligned}
& \nu \wedge g=\left(\left.u^{+}\right|_{\partial \Omega}\right)_{n o r}-\left(\left.u^{-}\right|_{\partial \Omega}\right)_{n o r}=: \widetilde{g} \in \nu \wedge L_{\text {tan }}^{p, \delta}\left(\partial \Omega, \mathcal{C l}_{4}\right), \\
& \nu \vee f=\left(\left.u^{+}\right|_{\partial \Omega}\right)_{\text {tan }}-\mu\left(\left.u^{-}\right|_{\partial \Omega}\right)_{\text {tan }}=: \widetilde{f} \in \nu \vee L_{\text {nor }}^{p, d}\left(\partial \Omega, \mathcal{C} \ell_{4}\right),
\end{aligned}
$$

which are the boundary conditions in Theorem 1.1 written for the boundary data $\widetilde{f}$ and $\widetilde{g}$ (in place of $f$ and $g$ ). Upon observing that one can also recover $g, f$ from $\widetilde{g}, \widetilde{f}$ by writing $g=\nu \vee \widetilde{g}$ and $f=\nu \wedge \widetilde{f}$, we may conclude that Theorem 7.1 implies Theorem 1.1. Finally, using (2.11), it follows that actually Theorem 1.1] is equivalent to Theorem [7.1. Hence, what we have proved so far in this section also justifies all the claims made in Theorem 1.1

\section{Connections with Maxwell's and Helmholtz's equations}

The main aim of this section is to understand the interplay between the transmission boundary value problem for the perturbed Dirac operator on the one hand, and the transmission boundary value problems for Maxwell's and Helmholtz's equations on the other hand. This section is divided into two parts and, in one direction, in Section 8.1 we start from the transmission boundary value problem for the perturbed Dirac operator and show that, in terms of the components of the functions involved, this contains several transmission boundary value problems for Maxwell's and Helmholtz's equations. The converse direction is studied in Section 8.2 , where we identify the necessary and sufficient conditions for the boundary data which guarantee that the transmission boundary value problem for the perturbed Dirac operator decouples into two independent transmission boundary value problems for Maxwell's equations.

8.1. Deconstructing the perturbed Dirac operator. Recall the transmission boundary value problem (7.1), and assume that $u^{ \pm} \in C^{1}\left(\Omega_{ \pm}, \mathrm{Cl}_{4}\right)$ solves this problem. Then (cf. (2.20) ), the functions $u^{ \pm}$can be canonically written as

$$
u^{ \pm}=U^{ \pm}-i e_{4} \odot \widetilde{U}^{ \pm},
$$

where $U^{ \pm}, \widetilde{U}^{ \pm} \in C^{1}\left(\Omega_{ \pm}, \mathcal{C} \ell_{3}\right)$. Let us further express $U^{ \pm}$and $\widetilde{U}^{ \pm}$by making use of the canonical decomposition (cf. (2.25) ), i.e.,

$$
\begin{aligned}
& U^{ \pm}=U_{0}^{ \pm}+* U_{0}^{\prime \pm}+U_{1}^{ \pm}+* U_{1}^{\prime}{ }^{ \pm}, \\
& \widetilde{U}^{ \pm}=\widetilde{U}_{0}^{ \pm}+* \widetilde{U}_{0}^{\prime \pm}+\widetilde{U}_{1}^{ \pm}+* \widetilde{U}_{1}^{\prime}{ }^{ \pm},
\end{aligned}
$$

and then combine all the above quantities in order to create the vectors

$$
\mathscr{U}^{ \pm}:=\left(U_{0}^{ \pm}, U_{1}^{ \pm}, U_{0}^{\prime \pm}, U_{1}^{\prime \pm}, \widetilde{U}_{0}^{ \pm}, \widetilde{U}_{1}^{ \pm}, \widetilde{U}_{0}^{\prime \pm}, \widetilde{U}_{1}^{\prime \pm}\right) .
$$

Lemma 8.1. Fix $k \in \mathbb{C}$. Then, with the above notation and conventions,

$$
\mathbb{D}_{k} u^{ \pm}=0 \quad \text { in } \Omega_{ \pm} \Longleftrightarrow D_{k} \mathscr{U}^{ \pm}=0 \text { in } \Omega_{ \pm},
$$


where $D_{k}$ is the first-order differential operator which is given by the $8 \times 8$ matrix

$$
D_{k}:=\left[\begin{array}{cccccccc}
0 & -\operatorname{div} & 0 & 0 & i k & 0 & 0 & 0 \\
\nabla & 0 & 0 & \text { curl } & 0 & i k & 0 & 0 \\
0 & \text { curl } & -\nabla & 0 & 0 & 0 & 0 & i k \\
0 & 0 & 0 & \operatorname{div} & 0 & 0 & i k & 0 \\
-i k & 0 & 0 & 0 & 0 & -\operatorname{div} & 0 & 0 \\
0 & -i k & 0 & 0 & \nabla & 0 & 0 & \text { curl } \\
0 & 0 & 0 & -i k & 0 & \text { curl } & -\nabla & 0 \\
0 & 0 & -i k & 0 & 0 & 0 & 0 & \operatorname{div}
\end{array}\right] .
$$

Proof. This can be verified in a straightforward fashion, using (2.26) and (2.27).

For further reference, let us note here that, componentwise, the two systems $D_{k} U^{ \pm}=0$ in $\Omega_{ \pm}$split into the following two sets of eight equations:

$$
\begin{aligned}
& -\operatorname{div} U_{1}^{ \pm}+i k \widetilde{U}_{0}^{ \pm}=0 \text { in } \Omega_{ \pm}, \\
& \nabla U_{0}^{ \pm}+\operatorname{curl} U_{1}^{\prime \pm}+i k \widetilde{U}_{1}^{ \pm}=0 \text { in } \Omega_{ \pm}, \\
& \operatorname{curl} U_{1}^{ \pm}-\nabla{U_{0}^{\prime \pm}}^{\prime \pm} \widetilde{U}_{1}^{\prime \pm}=0 \text { in } \Omega_{ \pm}, \\
& \operatorname{div} U_{1}^{\prime \pm}+i k \widetilde{U}_{0}^{\prime \pm}=0 \text { in } \Omega_{ \pm}, \\
& -i k U_{0}^{ \pm}-\operatorname{div} \widetilde{U}_{1}^{ \pm}=0 \text { in } \Omega_{ \pm}, \\
& -i k U_{1}^{ \pm}+\nabla \widetilde{U}_{0}^{ \pm}+\operatorname{curl} \widetilde{U}_{1}^{\prime \pm}=0 \text { in } \Omega_{ \pm}, \\
& -i k U_{1}^{\prime \pm}+\operatorname{curl} \widetilde{U}_{1}^{ \pm}-\nabla \widetilde{U}_{0}^{\prime \pm}=0 \text { in } \Omega_{ \pm}, \\
& -i k U_{0}^{\prime \pm}+\operatorname{div} \widetilde{U}_{1}^{\prime \pm}=0 \text { in } \Omega_{ \pm} .
\end{aligned}
$$

Lemma 8.2. Assume that $k \in \mathbb{C} \backslash\{0\}$ and consider four pairs of null-solutions of the Helmholtz operator, i.e., $w^{ \pm}, \widetilde{w}^{ \pm}, v^{ \pm}, \widetilde{v}^{ \pm} \in C^{\infty}\left(\Omega_{ \pm}\right)$satisfying

$$
\begin{gathered}
\left(\Delta+k^{2}\right) w^{ \pm}=0,\left(\Delta+k^{2}\right) \widetilde{w}^{ \pm}=0, \quad \text { in } \Omega_{ \pm} \quad \text { and } \\
\left(\Delta+k^{2}\right) v^{ \pm}=0,\left(\Delta+k^{2}\right) \widetilde{v}^{ \pm}=0 \quad \text { in } \Omega_{ \pm} .
\end{gathered}
$$

Next, define the vectors $\mathscr{U}^{ \pm}$as in (8.4) with components given in $\Omega_{ \pm}$by

$$
\begin{aligned}
& U_{0}^{ \pm}:=w^{ \pm}, \quad \widetilde{U}_{1}^{ \pm}:=i k^{-1} \nabla w^{ \pm}, \quad U_{1}^{\prime \pm}:=\nabla v^{ \pm}, \quad \widetilde{U}_{0}^{\prime \pm}:=i k v^{ \pm}, \\
& U_{1}^{ \pm}:=-\nabla \widetilde{w}^{ \pm}, \quad \widetilde{U}_{0}^{ \pm}:=-i k \widetilde{w}^{ \pm}, \quad \widetilde{U}_{1}^{\prime \pm}:=-i k^{-1} \nabla \widetilde{v}^{ \pm}, \quad U_{0}^{\prime \pm}:=\widetilde{v}^{ \pm} .
\end{aligned}
$$

Finally, define $u^{ \pm} \in C^{\infty}\left(\Omega_{ \pm}, \mathcal{C} l_{4}\right)$ as in (8.1) - (8.3). Then $\mathbb{D}_{k} u^{ \pm}=0$ in $\Omega_{ \pm}$. If, in addition, for some $p \in(1, \infty)$ one has

$$
\begin{gathered}
N\left(w^{ \pm}\right), N\left(\nabla w^{ \pm}\right), N\left(\widetilde{w}^{ \pm}\right), N\left(\nabla \widetilde{w}^{ \pm}\right) \in L^{p}(\partial \Omega) \text { and } \\
N\left(v^{ \pm}\right), N\left(\nabla v^{ \pm}\right), N\left(\widetilde{v}^{ \pm}\right), N\left(\nabla \widetilde{v}^{ \pm}\right) \in L^{p}(\partial \Omega),
\end{gathered}
$$

then also

$$
N\left(u^{ \pm}\right), N\left(\delta u^{ \pm}\right), N\left(d u^{ \pm}\right) \in L^{p}(\partial \Omega) .
$$

Finally, if $w^{-}, \widetilde{w}^{-}, v^{-}, \widetilde{v}^{-}$decay at infinity, then $u^{-}$also decays at infinity. 
Proof. That $\mathbb{D}_{k} u^{ \pm}=0$ in $\Omega_{ \pm}$follows from (8.7)-(8.14) and (8.15)-(8.16), after some straightforward algebra. It is also clear from (8.15)-(8.16) that (8.17) implies (8.18). Finally, the very last claim in the lemma is a consequence of Theorem 4.7 , (8.1)-(8.3), assumptions, and the fact that the derivatives of a null-solution of the Helmholtz equation which satisfies the Sommerfeld radiation condition continue to satisfy the Sommerfeld radiation condition (this can be seen using Green's representation formula [CK92, (2.8), p. 18] in terms of acoustic single and double layer potentials, the fact that these potentials satisfy the Sommerfeld radiation condition, as pointed out in [CK92, p. 38], and that the derivatives of these potentials may once again be expressed as a linear combination of acoustic single and double layer potentials).

Our next goal is to reinterpret the information encoded into the boundary conditions in (7.1). Making use of Theorem 3.5 and then further employing notation introduced in (3.17), we decompose $f \in L_{\text {nor }}^{p, d}\left(\partial \Omega, \mathcal{C l}_{4}\right)$ in the form

$$
\begin{gathered}
f=F+i e_{4} \odot \widetilde{F} \text { with } F, \widetilde{F} \in L_{\text {nor }}^{p, d}\left(\partial \Omega, \mathcal{C l}_{3}\right) \text { written as } \\
F=\nu F_{0}+* F_{1}+* F_{0}^{\prime} \quad \text { and } \widetilde{F}=\nu \widetilde{F_{0}}+* \widetilde{F_{1}}+* \widetilde{F_{0}^{\prime}}, \quad \text { where } \\
F_{0}, \widetilde{F_{0}} \in L_{1}^{p}(\partial \Omega), F_{1}, \widetilde{F_{1}} \in L_{\text {tan }}^{p, \operatorname{Div}}(\partial \Omega), F_{0}^{\prime}, \widetilde{F_{0}^{\prime}} \in L^{p}(\partial \Omega) .
\end{gathered}
$$

Similarly, making use of Corollary 3.4 and then using the notation introduced in (3.25), we express $g \in L_{t a n}^{p, \delta}\left(\partial \Omega, \mathcal{C l}_{4}\right)$ as

$$
\begin{gathered}
g=G+i e_{4} \odot \widetilde{G} \text { with } G, \widetilde{G} \in L_{\text {tan }}^{p, \delta}\left(\partial \Omega, C_{3}\right) \text { written as } \\
G=G_{0}+G_{1}+*\left(\nu G_{0}^{\prime}\right) \quad \text { and } \widetilde{G}=\widetilde{G_{0}}+\widetilde{G_{1}}+*\left(\nu \widetilde{G_{0}^{\prime}}\right), \quad \text { where } \\
G_{0}, \widetilde{G_{0}} \in L^{p}(\partial \Omega), G_{1}, \widetilde{G_{1}} \in L_{\text {tan }}^{p, \operatorname{Div}}(\partial \Omega), G_{0}^{\prime}, \widetilde{G_{0}^{\prime}} \in L_{1}^{p}(\partial \Omega) .
\end{gathered}
$$

In order to study the first boundary condition in (7.1), it is necessary to identify the components of $\nu \vee u^{ \pm}$. In this regard, (2.29), Lemma 2.6 and a simple calculation give that, on $\partial \Omega$,

$$
\begin{aligned}
\nu \vee u^{ \pm}= & \left\langle\nu, U_{1}^{ \pm}\right\rangle-\nu \times U_{1}^{\prime \pm}+*\left(\nu U_{0}^{\prime \pm}\right) \\
& +i e_{4} \odot\left[\left\langle\nu, \widetilde{U}_{1}^{ \pm}\right\rangle-\nu \times \widetilde{U}_{1}^{\prime \pm}+*\left(\nu \widetilde{U}_{0}^{\prime \pm}\right)\right] .
\end{aligned}
$$

Hence, by equating the components of $\nu \vee u^{+}-\nu \vee u^{-}$on $\partial \Omega$ with that of $g$, we conclude that the first boundary condition in (7.1) is equivalent to the following six equations on $\partial \Omega$ :

$$
\begin{aligned}
& \left\langle\nu,\left.U_{1}^{+}\right|_{\partial \Omega}\right\rangle-\left\langle\nu,\left.U_{1}^{-}\right|_{\partial \Omega}\right\rangle=G_{0}, \\
& -\nu \times\left. U_{1}^{\prime}\right|_{\partial \Omega}+\nu \times\left. U_{1}^{\prime-}\right|_{\partial \Omega}=G_{1}, \\
& \left.U_{0}^{\prime}\right|_{\partial \Omega}-U_{0}^{\prime}-\left.\right|_{\partial \Omega}=G_{0}^{\prime}, \\
& \left\langle\nu,\left.\widetilde{U}_{1}^{+}\right|_{\partial \Omega}\right\rangle-\left\langle\nu,\left.\widetilde{U}_{1}^{-}\right|_{\partial \Omega}\right\rangle=\widetilde{G_{0}}, \\
& -\nu \times\left.\widetilde{U}_{1}^{\prime}\right|_{\partial \Omega}+\nu \times\left.\widetilde{U}_{1}^{\prime}\right|_{\partial \Omega}=\widetilde{G_{1}}, \\
& \left.\widetilde{U}_{0}^{\prime+}\right|_{\partial \Omega}-\left.\widetilde{U}_{0}^{\prime-}\right|_{\partial \Omega}=\widetilde{G_{0}^{\prime}} .
\end{aligned}
$$


Similarly, it is not hard to check that, on $\partial \Omega$,

$$
\begin{aligned}
\nu \wedge u^{ \pm}= & \nu U_{0}^{ \pm}+*\left(\nu \times U_{1}^{ \pm}\right)+*\left\langle\nu, U_{1}^{\prime \pm}\right\rangle \\
& +i e_{4} \odot\left[\nu \widetilde{U}_{0}^{ \pm}+*\left(\nu \times \widetilde{U}_{1}^{ \pm}\right)+*\left\langle\nu, \widetilde{U}_{1}^{\prime}{ }^{\prime}\right\rangle\right] .
\end{aligned}
$$

Hence, by proceeding as above, we obtain that the second boundary condition in (7.1) is equivalent to the following six equations on $\partial \Omega$ :

$$
\begin{aligned}
& \left.U_{0}^{+}\right|_{\partial \Omega}-\left.\mu U_{0}^{-}\right|_{\partial \Omega}=F_{0}, \\
& \nu \times\left. U_{1}^{+}\right|_{\partial \Omega}-\mu \nu \times\left. U_{1}^{-}\right|_{\partial \Omega}=F_{1}, \\
& \left\langle\nu,\left.U_{1}^{\prime}\right|_{\partial \Omega}\right\rangle-\mu\left\langle\nu,\left.U_{1}^{\prime}\right|_{\partial \Omega}\right\rangle=F_{0}^{\prime}, \\
& \left.\widetilde{U}_{0}^{+}\right|_{\partial \Omega}-\left.\mu \widetilde{U}_{0}^{-}\right|_{\partial \Omega}=\widetilde{F_{0}}, \\
& \nu \times\left.\widetilde{U}_{1}^{+}\right|_{\partial \Omega}-\mu \nu \times\left.\widetilde{U}_{1}^{-}\right|_{\partial \Omega}=\widetilde{F_{1}}, \\
& \left\langle\nu,\left.\widetilde{U}_{1}^{\prime}\right|_{\partial \Omega}\right\rangle-\mu\left\langle\nu,\left.\widetilde{U}_{1}^{\prime}\right|_{\partial \Omega}\right\rangle=\widetilde{F_{0}^{\prime}} .
\end{aligned}
$$

Let us momentarily digress for the purpose of stating and proving a result which will shortly be of importance.

Lemma 8.3. Retain the context of Lemma 8.2 and assume that $\mu \in \mathbb{R}$ is arbitrary. Then the following statements are equivalent:

(1) There holds

$$
\left.\nu \vee u^{+}\right|_{\partial \Omega}-\left.\nu \vee u^{-}\right|_{\partial \Omega}=0 \text { and }\left.\quad \nu \wedge u^{+}\right|_{\partial \Omega}-\left.\mu \nu \wedge u^{-}\right|_{\partial \Omega}=0 .
$$

(2) One has

$$
\begin{aligned}
& \left\{\begin{array}{l}
\partial_{\nu} \widetilde{w}^{+}-\partial_{\nu} \widetilde{w}^{-}=0, \\
\left.\widetilde{w}^{+}\right|_{\partial \Omega}-\left.\mu \widetilde{w}^{-}\right|_{\partial \Omega}=0,
\end{array}\right. \\
& \text { and } \\
& \left\{\begin{array} { l } 
{ \partial _ { \nu } \widetilde { v } ^ { + } - \mu \partial _ { \nu } \widetilde { v } ^ { - } = 0 , } \\
{ \widetilde { v } ^ { + } | _ { \partial \Omega } - \widetilde { v } ^ { - } | _ { \partial \Omega } = 0 , }
\end{array} \quad \left\{\begin{array}{l}
\partial_{\nu} v^{+}-\mu \partial_{\nu} v^{-}=0, \\
\left.v^{+}\right|_{\partial \Omega}-\left.v^{-}\right|_{\partial \Omega}=0
\end{array}\right.\right. \\
&
\end{aligned}
$$

Proof. This follows directly from (8.16), (8.22)-(8.27), (8.29)-(8.34) and straightforward calculations.

Returning to the mainstream discussion, the idea is now to match partial differential equations from (8.7)-(8.14) with boundary conditions from (8.22)-(8.27) and (8.29)-(8.34) in a way which leads to recognizable boundary value problems. In this vein, note that by coupling the PDE's (8.13) and (8.8) with the boundary conditions (8.33) and (8.23) yields the inhomogeneous Maxwell system

$$
\left\{\begin{array}{l}
\operatorname{curl} \widetilde{U}_{1}^{ \pm}-i k U_{1}^{\prime \pm}=\nabla \widetilde{U}_{0}^{\prime \pm} \text { in } \Omega_{ \pm} \\
\operatorname{curl} U_{1}^{\prime \pm}+i k \widetilde{U}_{1}^{ \pm}=-\nabla U_{0}^{ \pm} \text {in } \Omega_{ \pm} \\
\nu \times\left.\widetilde{U}_{1}^{+}\right|_{\partial \Omega}-\mu \nu \times\left.\widetilde{U}_{1}^{-}\right|_{\partial \Omega}=\widetilde{F}_{1} \\
\nu \times\left. U_{1}^{\prime}\right|_{\partial \Omega}-\nu \times U_{1}^{\prime}-\left.\right|_{\partial \Omega}=-G_{1} \\
U_{1}^{\prime}, \widetilde{U}_{1}^{-} \text {decay at infinity, }
\end{array}\right.
$$


where the above decay condition (cf. the discussion in Section 11) is a consequence of Theorem 4.7 and the fact that $u^{-}$decays at infinity. On the other hand, putting together the PDE's (8.12) and (8.9) with the boundary conditions (8.30) and (8.26) gives rise to a second inhomogeneous Maxwell system, namely

$$
\left\{\begin{array}{l}
\operatorname{curl} \widetilde{U}_{1}^{ \pm}-i k U_{1}^{ \pm}=-\nabla \widetilde{U}_{0}^{ \pm} \text {in } \Omega_{ \pm} \\
\operatorname{curl} U_{1}^{ \pm}+i k \widetilde{U}_{1}^{\prime \pm}=\nabla U_{0}^{\prime} \pm \text { in } \Omega_{ \pm} \\
\nu \times\left. U_{1}^{+}\right|_{\partial \Omega}-\mu \nu \times\left. U_{1}^{-}\right|_{\partial \Omega}=F_{1} \\
\nu \times\left.\widetilde{U}_{1}^{\prime}\right|_{\partial \Omega}-\nu \times \widetilde{U}_{1}^{\prime}-\left.\right|_{\partial \Omega}=-\widetilde{G_{1}} \\
U_{1}^{-}, \widetilde{U}_{1}^{\prime}-\text { decay at infinity. }
\end{array}\right.
$$

So far, the process of converting parts of (8.7)-(8.14) and (8.22)-(8.27), (8.29)(8.34) into the two inhomogeneous Maxwell systems (8.38)-(8.39) has been carried out without loss of information (since all steps are obviously reversible). Executing a similar procedure for the remaining PDE's and boundary conditions turns out to be a one-way process leading to a number of boundary value problems for the Helmholtz operator $\Delta+k^{2}$. We first focus on understanding the nature of the expression $\partial_{\nu} U_{0}^{+}-\partial_{\nu} U_{0}^{-}$which, given that $\partial_{\nu}$ stands for the normal derivative operator, can be written as

$$
\partial_{\nu} U_{0}^{+}-\partial_{\nu} U_{0}^{-}=\left\langle\nu,\left.\nabla U_{0}^{+}\right|_{\partial \Omega}\right\rangle-\left\langle\nu,\left.\nabla U_{0}^{-}\right|_{\partial \Omega}\right\rangle .
$$

From (8.8) we have $\nabla U_{0}^{+}=-i k \widetilde{U}_{1}^{+}-\operatorname{curl}{U_{1}^{\prime}}^{+}$, and, generally speaking, the identity $\left\langle\nu,\left.(\operatorname{curl} U)\right|_{\partial \Omega}\right\rangle=-\operatorname{Div}\left(\nu \times\left. U\right|_{\partial \Omega}\right)$ holds for any reasonably behaved vector field $U$. Therefore,

$$
\begin{aligned}
\left\langle\nu,\left.\nabla U_{0}^{+}\right|_{\partial \Omega}\right\rangle & =-i k\left\langle\nu,\left.\widetilde{U}_{1}^{+}\right|_{\partial \Omega}\right\rangle-\left\langle\nu,\left.\left(\operatorname{curl} U_{1}^{\prime+}\right)\right|_{\partial \Omega}\right\rangle \\
& =-\left.i k\left\langle\nu, \widetilde{U}_{1}^{+}\right\rangle\right|_{\partial \Omega}+\operatorname{Div}\left(\nu \times\left. U_{1}^{\prime}\right|_{\partial \Omega}\right) .
\end{aligned}
$$

Similarly,

$$
\left\langle\nu,\left.\nabla U_{0}^{-}\right|_{\partial \Omega}\right\rangle=-i k\left\langle\nu,\left.\widetilde{U}_{1}^{-}\right|_{\partial \Omega}\right\rangle+\operatorname{Div}\left(\nu \times\left. U_{1}^{\prime}\right|_{\partial \Omega}\right) .
$$

Using (8.41) and (8.42) into (8.40) then gives

$$
\begin{aligned}
\partial_{\nu} U_{0}^{+}-\partial_{\nu} U_{0}^{-}= & -i k\left\langle\nu,\left.\widetilde{U}_{1}^{+}\right|_{\partial \Omega}\right\rangle+i k\left\langle\nu,\left.\widetilde{U}_{1}^{-}\right|_{\partial \Omega}\right\rangle+\operatorname{Div}\left(\nu \times U_{1}^{\prime}+\left.\right|_{\partial \Omega}\right) \\
& -\operatorname{Div}\left(\nu \times\left. U_{1}^{\prime}\right|_{\partial \Omega}\right) \\
= & -i k\left\langle\nu,\left.\left(\widetilde{U}_{1}^{+}-\widetilde{U}_{1}^{-}\right)\right|_{\partial \Omega}\right\rangle-\operatorname{Div}\left(\nu \times\left.\left(U_{1}^{\prime}-U_{1}^{\prime}\right)\right|_{\partial \Omega}\right) .
\end{aligned}
$$

Having established this and making use of the boundary conditions (8.25) and (8.23), we finally arrive at the conclusion that, on $\partial \Omega$,

$$
\partial_{\nu} U_{0}^{+}-\partial_{\nu} U_{0}^{-}=-i k \widetilde{G_{0}}-\operatorname{Div} G_{1}
$$

Since any component of a $k$-monogenic function is a null-solution for the Helmholtz operator which, by Theorem 4.7, also decays at infinity if the $k$-monogenic function in question is defined in the complement of a compact set and decays at infinity, 
and keeping in mind the boundary conditions (8.29) and (8.44), we then obtain the following transmission boundary value problem for the Helmholtz operator:

$$
\left\{\begin{array}{l}
\left(\Delta+k^{2}\right) U_{0}^{ \pm}=0 \text { in } \Omega_{ \pm}, \\
\left.U_{0}^{+}\right|_{\partial \Omega}-\left.\mu U_{0}^{-}\right|_{\partial \Omega}=F_{0}, \\
\partial_{\nu} U_{0}^{+}-\partial_{\nu} U_{0}^{-}=-i k \widetilde{G_{0}}-\operatorname{Div} G_{1}, \\
U_{0}^{-} \text {decays at infinity. }
\end{array}\right.
$$

By proceeding in a similar fashion, we also obtain three other transmission boundary value problems for the Helmholtz operator which are satisfied by components of $U^{ \pm}$, namely,

$$
\left\{\begin{array}{l}
\left(\Delta+k^{2}\right) \widetilde{U}_{0}^{ \pm}=0 \text { in } \Omega_{ \pm}, \\
\left.\widetilde{U}_{0}^{+}\right|_{\partial \Omega}-\left.\mu \widetilde{U}_{0}^{-}\right|_{\partial \Omega}=\widetilde{F}_{0}, \\
\partial_{\nu} \widetilde{U}_{0}^{+}-\partial_{\nu} \widetilde{U}_{0}^{-}=i k G_{0}-\operatorname{Div} \widetilde{G_{1}}, \\
\widetilde{U}_{0}^{-} \text {decays at infinity, }
\end{array}\right.
$$

$$
\left\{\begin{array}{l}
\left(\Delta+k^{2}\right) U_{0}^{\prime \pm}=0 \text { in } \Omega_{ \pm}, \\
\left.U_{0}^{\prime}\right|_{\partial \Omega}-U_{0}^{\prime}-\left.\right|_{\partial \Omega}=G_{0}^{\prime}, \\
\partial_{\nu} U_{0}^{\prime}-\mu \partial_{\nu} U_{0}^{\prime}-i k \widetilde{F_{0}^{\prime}}-\operatorname{Div} F_{1}, \\
U_{0}^{\prime}-\text { decays at infinity, }
\end{array}\right.
$$

and

$$
\left\{\begin{array}{l}
\left(\Delta+k^{2}\right) \widetilde{U}_{0}^{\prime}=0 \text { in } \Omega_{ \pm}, \\
\widetilde{U}_{0}^{\prime}+\left.\right|_{\partial \Omega}-\widetilde{U}_{0}^{\prime}-\left.\right|_{\partial \Omega}=\widetilde{G_{0}^{\prime}}, \\
\partial_{\nu} \widetilde{U}_{0}^{\prime}-\mu \partial_{\nu} \widetilde{U}_{0}^{\prime}=-i k F_{0}^{\prime}-\operatorname{Div} \widetilde{F_{1}}, \\
\widetilde{U}_{0}^{\prime}-\text { decays at infinity. }
\end{array}\right.
$$

All in all, the above analysis proves the following theorem.

Theorem 8.4. The transmission boundary value problem (7.1) contains four transmission boundary value problems for the Helmholtz operator and two inhomogeneous transmission boundary value problems for Maxwell's system, in the following precise sense. Suppose that $\Omega$ is a bounded Lipschitz domain in $\mathbb{R}^{3}$, and fix $\mu \in(0,1)$, $k \in \mathbb{C}$ and $p \in(1, \infty)$. Also, assume that $u^{ \pm} \in C^{1}\left(\Omega_{ \pm}, \mathcal{C l}_{4}\right)$ solves the transmission boundary value problem for the perturbed Dirac operator (7.1). Finally, decompose $u^{ \pm}$as in (8.1) -(8.3), and also decompose the boundary data $f, g$ as in (8.19) -(8.20). Then the components of these functions satisfy (8.38)-(8.39) and (8.45)-(8.48).

Corollary 8.5. Assume that $\Omega$ is a bounded Lipschitz domain in $\mathbb{R}^{3}$. Then there exist $\varepsilon>0$ and an at most countable set of real numbers $\left\{k_{j}\right\}_{j}$ which depend exclusively on $\partial \Omega$ such that if $p \in(1,2+\varepsilon), k \in \mathbb{C} \backslash\left\{k_{j}\right\}_{j}$ has $\operatorname{Im} k \geq 0$, and $\mu \in(0,1)$, then the Helmholtz transmission problem (1.7) is well-posed. 
Proof. Let $\varepsilon>0$ and $\left\{k_{j}\right\}_{j}$ be as in Theorem 7.1 and assume that $p, k, \mu$ are as above. Then, given $\phi \in L_{1}^{p}(\partial \Omega)$ and $\psi \in L^{p}(\partial \Omega)$ arbitrary, take

$$
f:=\nu \phi \in L_{\text {nor }}^{p, d}\left(\partial \Omega, \mathcal{C l}_{4}\right), \quad g:=-k^{-1} e_{4} \odot \psi \in L_{\text {tan }}^{p, \delta}\left(\partial \Omega, \mathcal{C l}_{4}\right),
$$

and then let $u^{ \pm}$be the solution of Dirac transmission problem (7.1) with data (8.49). Finally, decompose $u^{ \pm}$as in (8.1) - (8.3). Then, thanks to (8.45), $w^{ \pm}:=$ $U_{0}^{ \pm}$solves the Helmholtz transmission problem with data $\phi, \psi$, with appropriate estimates. As far as the uniqueness for the Helmholtz transmission problem is concerned, this follows from Lemma 8.2. Lemma 8.3 and the uniqueness portion of Theorem 7.1

Theorem 8.4 sheds light on the structure of the transmission boundary value problem (7.1) for the perturbed Dirac operator $\mathbb{D}_{k}$ by identifying several transmission problems for the Maxwell and Helmholtz equations which are satisfied by the components of the original solution of (7.1). In particular, as discussed in Corollary 8.5, the well-posedness of (7.1) implies the solvability of the Helmholtz transmission problem (1.7). Matters are more subtle as far as the Maxwell transmission problem is concerned, given that (8.38)-(8.39) are inhomogeneous problems. As such, one important aspect is to single out a subclass of problems of the type (7.1), identified by the demand that the boundary data $f, g$ are of a certain specific nature, which allows one to equivalently decouple (17.1) into two independent Maxwell transmission problems.

8.2. Connections with the transmission problems for Maxwell's equations. The aim here is to prove the following theorem, addressing the issue raised at the end of the previous subsection.

Theorem 8.6. For each bounded Lipschitz domain $\Omega$ in $\mathbb{R}^{3}$ there exist $\varepsilon>0$ and an at most countable set of real numbers $\left\{k_{j}\right\}_{j}$ having the following significance. Whenever $\mu \in(0,1), 1<p<2+\varepsilon$ and $k \in \mathbb{C} \backslash\left\{k_{j}\right\}_{j}$ has $\operatorname{Im} k \geq 0$, the Dirac transmission boundary value problem (7.1), with $u^{ \pm}$written as in (8.1)-(8.3), $g$ written as in (8.20) and $f$ written as in (8.19), decouples into the following two independent transmission problems for the Maxwell's systems:

$$
\left\{\begin{array}{l}
\operatorname{curl} \widetilde{U}_{1}^{ \pm}-i k U_{1}^{\prime \pm}=0 \text { in } \Omega_{ \pm} \\
\operatorname{curl} U_{1}^{\prime \pm}+i k \widetilde{U}_{1}^{ \pm}=0 \text { in } \Omega_{ \pm} \\
\nu \times\left.\widetilde{U}_{1}^{+}\right|_{\partial \Omega}-\mu \nu \times\left.\widetilde{U}_{1}^{-}\right|_{\partial \Omega}=\widetilde{F}_{1} \in L_{t a n}^{p, \text { Div }}(\partial \Omega), \\
\nu \times\left. U_{1}^{\prime}\right|_{\partial \Omega}-\nu \times U_{1}^{\prime}-\left.\right|_{\partial \Omega}=-G_{1} \in L_{\text {tan }}^{p, \text { Div }}(\partial \Omega), \\
U_{1}^{\prime}-\widetilde{U}_{1}^{-} \text {decay at infinity }
\end{array}\right.
$$

and

$$
\left\{\begin{array}{l}
\operatorname{curl} U_{1}^{ \pm}+i k \widetilde{U}_{1}^{\prime \pm}=0 \text { in } \Omega_{ \pm}, \\
\operatorname{curl} \widetilde{U}_{1}^{\prime \pm}-i k U_{1}^{ \pm}=0 \text { in } \Omega_{ \pm}, \\
\nu \times\left. U_{1}^{+}\right|_{\partial \Omega}-\mu \nu \times\left. U_{1}^{-}\right|_{\partial \Omega}=F_{1} \in L_{t a n}^{p, \text { Div }}(\partial \Omega), \\
\nu \times\left.\widetilde{U}_{1}^{\prime+}\right|_{\partial \Omega}-\nu \times\left.\widetilde{U}_{1}^{\prime}\right|_{\partial \Omega}=-\widetilde{G}_{1} \in L_{t a n}^{p, \text { Div }}(\partial \Omega), \\
U_{1}^{-}, \widetilde{U}_{1}^{\prime}-\text { decay at infinity }
\end{array}\right.
$$


if and only if

$$
\begin{gathered}
d_{\partial} f+k e_{4} \odot f \text { is }\left(\Lambda^{2} \oplus\left(e_{4} \odot \Lambda^{2}\right)\right) \text {-valued and } \\
\delta_{\partial} g+k e_{4} \odot g \text { is }\left(\Lambda^{1} \oplus\left(e_{4} \odot \Lambda^{1}\right)\right) \text {-valued. }
\end{gathered}
$$

Furthermore, in the case when (8.52) holds, the connections between the boundary data for the transmission problem for the perturbed Dirac operator (7.1) and the boundary data for the transmission problem for the two Maxwell systems (8.50)(8.51) read

$(8.53) *\left(d_{\partial} f+k e_{4} \odot f\right)=-i k \widetilde{F_{1}}+k e_{4} \odot F_{1}, \quad \delta_{\partial} g+k e_{4} \odot g=-i k \widetilde{G_{1}}+k e_{4} \odot G_{1}$, which determine the vector fields $\widetilde{F_{1}}, F_{1}, \widetilde{G_{1}}, G_{1}$ in terms of $f, g$ and, in the converse direction,

$$
\begin{aligned}
& f=* F_{1}+i k^{-1} *\left(\operatorname{Div} \widetilde{F_{1}}\right)+i e_{4} \odot\left(* \widetilde{F_{1}}-i k^{-1} *\left(\operatorname{Div} F_{1}\right)\right), \\
& g=-i k^{-1} \operatorname{Div} \widetilde{G_{1}}+G_{1}+i e_{4} \odot\left(i k^{-1} \operatorname{Div} G_{1}+\widetilde{G_{1}}\right) .
\end{aligned}
$$

Proof. The first step is to observe, with the help of Theorem 8.4 that the transmission boundary value problem (7.1) is equivalent to the two Maxwell transmission boundary value problems (8.50)-(8.51) if and only if the functions $U_{0}^{ \pm}, U_{0}^{\prime}, \widetilde{U}_{0}^{ \pm}$ and $\widetilde{U}_{0}^{\prime \pm}$ vanish identically. On the other hand, due to the well-posedness of the Helmholtz transmission boundary value problems (8.45)-(8.48) (cf. Corollary 8.5), the functions $U_{0}^{ \pm}, U_{0}^{\prime \pm}, \widetilde{U}_{0}^{ \pm}$and $\widetilde{U}_{0}^{\prime \pm}$ are zero if and only if the boundary data for these four Helmholtz transmission problems are zero. The bottom line is that it suffices to show that the boundary data of the four aforementioned boundary value problems are zero if and only if (8.52) holds. With this strategy in mind, we begin by computing

$$
d_{\partial} f+k e_{4} \odot f=\left(d_{\partial} F-i k \widetilde{F}\right)-i e_{4} \odot\left(d_{\partial} \widetilde{F}+i k F\right),
$$

with $d_{\partial} F-i k \widetilde{F}$ and $d_{\partial} \widetilde{F}+i k F$ both $\mathrm{Cl}_{3}$-valued. Next, upon recalling (3.23), we write

$$
\begin{aligned}
d_{\partial} F-i k \widetilde{F}= & -i k \nu \widetilde{F_{0}}-*\left(\nu \times \nabla_{t a n} F_{0}\right)-i k\left(* \widetilde{F_{1}}\right) \\
& +*\left(\operatorname{Div} F_{1}-i k \widetilde{F_{0}^{\prime}}\right),
\end{aligned}
$$

which shows that the $\Lambda^{1}$-component of (8.57) is $-i k \nu \widetilde{F_{0}}$, while its $\Lambda^{3}$-component is given by $*\left(\operatorname{Div} F_{1}-i k \widetilde{F_{0}^{\prime}}\right)$. As a consequence, the $\Lambda^{1}$ - and $\Lambda^{3}$-components of $d_{\partial} F-i k \widetilde{F}$ are zero if and only if

$$
\widetilde{F_{0}}=0 \text { and } i k \widetilde{F_{0}^{\prime}}-\operatorname{Div} F_{1}=0 \text { on } \partial \Omega .
$$

The incisive observation now is that these expressions, i.e., $\widetilde{F_{0}}$ and $i k \widetilde{F_{0}^{\prime}}-\operatorname{Div} F_{1}$, make up the first boundary datum in the problem $(\underline{8.46})$ and the second boundary datum in the problem (8.47), respectively. By arguing in a similar fashion in the case of $e_{4} \odot\left(d_{\partial} \widetilde{F}+i k F\right)$, we see that the $\Lambda^{1}$ - and $\Lambda^{3}$-components of this function vanish if and only if

$$
F_{0}=0 \text { and }-i k F_{0}^{\prime}-\operatorname{Div} \widetilde{F_{1}}=0 \text { on } \partial \Omega,
$$

i.e., the second boundary datum in the problem (8.46), as well as the first boundary datum in the problem (8.47), vanish. In summary, the function $d_{\partial} f+k e_{4} \odot f$ is $\left(\Lambda^{2} \oplus\left(e_{4} \odot \Lambda^{2}\right)\right)$-valued if and only if the four boundary conditions mentioned 
above are all zero. In a similar manner, by decomposing the boundary datum $g \in L_{\text {tan }}^{p, \delta}\left(\partial \Omega, \mathcal{C l}_{4}\right)$, one can check that $\delta_{\partial} g+k e_{4} \odot g$ is $\left(\Lambda^{1} \oplus\left(e_{4} \odot \Lambda^{1}\right)\right)$-valued if and only if

$$
\begin{array}{lllll}
G_{0}^{\prime}=0 & \text { and } & -i k \widetilde{G_{0}}-\operatorname{Div} G_{1}=0 & \text { on } & \partial \Omega, \\
\widetilde{G_{0}^{\prime}}=0 & \text { and } \quad i k G_{0}-\operatorname{Div} \widetilde{G_{1}}=0 & \text { on } & \partial \Omega,
\end{array}
$$

i.e., when the other four remaining boundary conditions in the boundary value problems (8.45)-(8.48) are zero. Moving on, the first formula in (8.53) is seen from (8.56)-(8.59), whereas the second formula in (8.53) is proved in an analogous fashion. Finally, (8.54) follows from (8.19) and (8.58)-(8.59), while (8.55) is a consequence of (8.20) and (8.60)-(8.61).

Our next result establishes a direct link between the Dirac transmission problem and just one Maxwell transmission problem.

Corollary 8.7. In the context of Theorem 8.6, the transmission boundary value problem (7.1) reduces to just one transmission boundary value problem for the Maxwell system if and only if the boundary data $f, g$ are $\left(\Lambda^{3} \oplus\left(e_{4} \odot \Lambda^{2}\right)\right)$-valued and $\left(\Lambda^{1} \oplus\left(e_{4} \odot \Lambda^{0}\right)\right)$-valued, respectively, and satisfy

$$
d_{\partial} f-k e_{4} \wedge f=0 \quad \text { and } \quad \delta_{\partial} g-k e_{4} \vee g=0 .
$$

More specifically, the Dirac transmission problem (7.1) reduces precisely to the Maxwell transmission problem (8.50) if and only if

$$
\begin{gathered}
f=i k^{-1} *\left(\operatorname{Div} \widetilde{F_{1}}\right)+i e_{4} \odot\left(* \widetilde{F_{1}}\right) \quad \text { and } \\
g=G_{1}-k^{-1} e_{4} \odot\left(\operatorname{Div} G_{1}\right) .
\end{gathered}
$$

Proof. Taking $F_{1}=0$ and $\widetilde{G_{1}}=0$ in Theorem 8.6 proves, due to 8.54 - 8.55 , that the Dirac transmission problem (7.1) reduces precisely to the Maxwell transmission problem (8.50) if and only if (8.63) holds. It is also not difficult to check that if $f, g$ are as in (8.63), then the function $f$ is $\left(\Lambda^{3} \oplus\left(e_{4} \odot \Lambda^{2}\right)\right)$-valued, the function $g$ is $\left(\Lambda^{1} \oplus\left(e_{4} \odot \Lambda^{0}\right)\right)$-valued, and (8.62) holds.

Conversely, if $f$ is $\left(\Lambda^{3} \oplus\left(e_{4} \odot \Lambda^{2}\right)\right)$-valued, $g$ is $\left(\Lambda^{1} \oplus\left(e_{4} \odot \Lambda^{0}\right)\right)$-valued, and (8.62) holds, then

$$
\begin{aligned}
d_{\partial} f+k e_{4} \odot f & =k\left(e_{4} \odot f-e_{4} \wedge f\right) \\
& =k e_{4} \vee f \in \Lambda^{2} \hookrightarrow \Lambda^{2} \oplus\left(e_{4} \odot \Lambda^{2}\right), \\
\delta_{\partial} g+k e_{4} \odot g & =k\left(e_{4} \odot g-e_{4} \vee g\right) \\
& =k e_{4} \wedge g \in e_{4} \odot \Lambda^{2} \hookrightarrow \Lambda^{1} \oplus\left(e_{4} \odot \Lambda^{1}\right) .
\end{aligned}
$$

In concert with Theorem 8.6, formulas 8.64)-(8.65) show that the Dirac transmission problem (7.1) reduces to the two Maxwell transmission problems listed in (8.50), (8.51). As a consequence, formulas (8.54), (8.54) represent, respectively, $\left(\Lambda^{3} \oplus\left(e_{4} \odot \Lambda^{2}\right)\right)$-valued and $\left(\Lambda^{1} \oplus\left(e_{4} \odot \Lambda^{0}\right)\right)$-valued functions. In turn, this forces $F_{1}=0$ and $\widetilde{G_{1}}=0$, which shows that, in fact, the Dirac transmission problem (7.1) reduces precisely to just the Maxwell transmission problem (8.50).

From the above results, the following algorithm emerges for solving the transmission boundary value problem (8.50) for the Maxwell system, granted the wellposedness of the transmission boundary value problem (7.1) for the perturbed Dirac 
operator. Given two functions $\widetilde{F_{1}}, G_{1} \in L_{\text {tan }}^{p \text {,Div }}(\partial \Omega)$, define $f \in L_{\text {nor }}^{p, d}\left(\partial \Omega, C \ell_{4}\right)$ and $g \in L_{\text {tan }}^{p, \delta}\left(\partial \Omega, \mathcal{C l}_{4}\right)$ as in (8.63), and denote by $u^{ \pm}$the solution of (7.1) corresponding to the boundary data $f, g$. If we now decompose $u^{ \pm}$as in (8.1)-(8.3), then $\widetilde{U}_{1}^{ \pm}, U_{1}^{\prime} \pm$ is the unique solution of the transmission boundary value problem (8.50) for the Maxwell system. In particular, in the setting of Theorem 8.6, the Maxwell transmission problem (1.4) is well-posed.

\section{ACKNOWLEDGMENTS}

The first author wishes to thank Salvador Pérez-Esteva for several fruitful discussions. The work of the second author has been supported in part by the US NSF DMS CAREER grant 1048467. Part of this work was done when the third author visited the Instituto de Matemáticas Unidad Cuernavaca, while on research leave from the University of Missouri. He gratefully acknowledges the support received from these institutions, as well as the support through US NSF grant DMS-0653180. Last but not least, the authors thank the referees for their careful reading of the manuscript and for making several useful suggestions.

\section{REFERENCES}

[AS72] M. Abramowitz and I.A. Stegun, Handbook of Mathematical Functions, Dover, New York, 1972.

[Ag97] M.S. Agranovich, Elliptic boundary problems, Encyclopaedia Math. Sci., 79, Partial differential equations, IX, pp. 1-144 and pp. 275-281, Springer, Berlin, 1997. MR.1481215 (99a:35056)

[AK92] T. Angell and A. Kirsch, The conductive boundary condition for Maxwell's equations, SIAM J. Appl. Math., 52 (1992), 1597-1610. MR.1191352 (93k:78009)

[BD54] R.B. Barrar and C.L. Dolph, On a three dimensional transmission problem of electromagnetic theory, J. Rational Mech. Anal., 3 (1954), 725-743. MR0064639 (16:313f)

[BDS82] F. Brackx, R. Delanghe and F. Sommen, Clifford Analysis, Research Notes in Mathematics, Vol. 76, Pitman, Boston, MA, 1982. MR697564 (85j:30103)

[Br89] R. Brown, The method of layer potentials for the heat equation in Lipschitz cylinders, Amer. J. Math., 111 (1989), 339-379. MR987761 (90d:35118)

[Ca54] A. Calderón, The multipole expansion of radiation fields, J. Rat. Mech. Anal., 3 (1954), 523-537. MR0063540(16:136a)

[Ca77] A.P. Calderón, Cauchy integrals on Lipschitz curves and related operators, Proc. Nat. Acad. Sci. USA., 74 (1977), no. 4, 1324-1327. MR0466568(57:6445)

[Ca80] A.P. Calderón, Commutators, singular integrals on Lipschitz curves and applications, pp. 85-96 in Proceedings of the International Congress of Mathematicians (Helsinki, 1978), Acad. Sci. Fennica, Helsinki, 1980. MR.562599 (82f:42016)

[CK83] D. Colton and R. Kress, Integral Equation Methods in Scattering Theory, Wiley Interscience Publications, New York, 1983. MR700400 (85d:35001)

[CK92] D. Colton and R. Kress, Inverse Acoustic and Electromagnetic Scattering Theory, Springer-Verlag, Applied Math. Series, No. 93, 1992. MR1183732 (93j:35124)

[CMM82] R. Coifman, A. McIntosh, and Y. Meyer, L'intégrale de Cauchy definit un opérateur borné sur $L^{2}$ pour les courbes Lipschitziennes, Annals of Math., 116 (1982), 361-388. MR672839 (84m:42027)

[Da77] J. E. Dahlberg, On estimates of harmonic measure, Arch. Rat. Mech. Anal., 65 (1977), 275-288. MR0466593 (57:6470)

[DK87] B. Dahlberg and C. Kenig, Hardy spaces and the $L^{p}-$ Neumann problem for Laplace's equation in a Lipschitz domain, Ann. of Math., 125 (1987), 437-465. MR890159 (88d:35044)

[DK90] B.E.J.Dahlberg and C.E.Kenig, $L^{p}$ estimates for the three-dimensional systems of elastostatics on Lipschitz domains, Analysis and Partial Differential Equations, pp.621-634, Lecture Notes in Pure and Appl. Math., Vol.122, Dekker, New York, 1990. MR1044810 (91h:35053) 
[DKV88] B.E.J. Dahlberg, C.E. Kenig and G.C. Verchota, Boundary value problems for the systems of elastostatics in Lipschitz domains, Duke Math. J., 57 (1988), no. 3, 795-818. MR.975122 (90d:35259)

[DL90] R. Dautray and J.-L. Lions, Mathematical Analysis and Numerical Methods for Science and Technology, Vol. 4, Springer-Verlag, 1990. MR1081946 (91h:00004b)

[DMM06] R. Duduchava, D. Mitrea and D. Mitrea, Differential operators and boundary value problems on surfaces, Mathematische Nachrichten, 9-10 (2006), 996-1023.

[EFV92] L. Escauriaza, E. Fabes and G. Verchota, On a regularity theorem for weak solutions to transmission problems with internal Lipschitz boundaries, Proc. Amer. Math. Soc., 115 (1992), 1069-1076. MR.1092919 (92j:35020)

[EM04] L. Escauriaza and M. Mitrea, Transmission problems and spectral theory for singular integral operators on Lipschitz domains, J. Funct. Anal., 216 (2004), no. 1, 141-171. MR2091359 (2005f:35065)

[ES93] L. Escauriaza and J.K. Seo, Regularity properties of solutions to transmission problems, Trans. Amer. Math. Soc., 338 (1993), 405-430. MR1149120 (93j:35039)

[FJR78] E. Fabes, M. Jodeit and N. Rivière, Potential techniques for boundary value problems on $C^{1}$ domains, Acta Math., 141 (1978), 165-186. MR.501367 (80b:31006)

[FKV88] E.B. Fabes, C.E. Kenig and G.C. Verchota, The Dirichlet problem for the Stokes system on Lipschitz domains, Duke Math. J., 57 (1988), no. 3, 769-793. MR975121 (90d:35258)

[GK96] T. Gerlach and R. Kress, Uniqueness in inverse obstacle scattering with conductive boundary condition, Inverse Problems, 12 (1996), 619-625. MR.1413422 (97i:35184a)

[GeMi08] F. Gesztesy and M. Mitrea, Generalized Robin boundary conditions, Robin-toDirichlet maps, and Krein-type resolvent formulas for Schrödinger operators on bounded Lipschitz domains, pp. 105-173 in the Proceedings of Symposia in Pure Mathematics, Vol. 79, Amer. Math. Soc., 2008. MR2500491 (2010k:35087)

[GM91] J. Gilbert and M.A. Murray, Clifford Algebras and Dirac Operators in Harmonic Analysis, Cambridge Studies in Advanced Mathematics, 26, Cambridge Univ. Press, Cambridge 1991. MR:1130821 (93c:42027)

[H-T09] S. Hofmann, E. Marmolejo-Olea, M. Mitrea, S. Perez-Esteva, and M. Taylor, Hardy spaces, singular integrals and the geometry of Euclidean domains of locally finite perimeter, Journal Geometric and Functional Analysis, 19, (2009), no. 3, 842-882. MR2563770(2011a:42021)

[HMT10] S. Hofmann, M. Mitrea, and M. Taylor, Singular integrals and elliptic boundary problems on regular Semmes-Kenig-Toro domains, International Mathematics Research Notices, Oxford University Press, 2010 (14), 2567-2865. MR2669659

[JM95] B. Jawerth and M. Mitrea, Higher dimensional scattering theory on $C^{1}$ and Lipschitz domains, Amer. J. of Math., 117 (1995), 929-963. MR.1342836 (96h:35143)

[Ke94] C.E. Kenig, Harmonic Analysis Techniques for Second Order Elliptic Boundary Value Problems, CBMS Regional Conference Series in Mathematics, No. 83, AMS, Providence, RI, 1994. MR:1282720 (96a:35040)

[KM88] R.E.Kleinman and P.A. Martin, On single integral equations for the transmission problem of acoustics, SIAM J. Appl. Math., 48 (1988), 307-325. MR933037 (89f:35053)

[KP98] A. Kirsch and L. Päivärinta, On recovering obstacles inside inhomogeneities, Math. Methods Appl. Sci., 21 (1998), 619-651. MR1615992 (99b:35214)

[KMR01] V.A. Kozlov, V.G. Maz'ya and J. Rossmann, Spectral Problems Associated with Corner Singularities of Solutions to Elliptic Equations, Amer. Math. Soc., Providence, RI, 2001. MR.1788991 (2001i:35069)

[La93] S. Lang, Real and Function Analysis, third edition, Springer-Verlag, 1993. MR.1216137 (94b:00005)

[LRU66] O.A. Ladyzenskaja, V.J.Rivkind and N.N. Ural'ceva, The classical solvability of diffraction problems, Proc. Steklov Inst. Math., 92 (1966), 132-166. MR0211050 (35:1932)

[MM03] E. Marmolejo-Olea and M. Mitrea, Harmonic analysis for general first order differential operators in Lipschitz domains, pp. 91-114 in "Clifford Algebras: Application to Mathematics, Physics, and Engineering", Birkhäuser Progress in Mathematical Physics Series, 2003. MR2025974 (2004k:58033) 
[MO93] P.A. Martin and P. Ola, Boundary integral equations for the scattering of electromagnetic waves by a homogeneous dielectric obstacle, Proc. of the Royal Soc. of Edinburgh, 123 (1993), 185-208. MR1204856 (94c:78008)

[McMM97] A. McIntosh, D. Mitrea and M. Mitrea, Rellich type identities for one-sided monogenic functions in Lipschitz domains and applications, pp. 135-143 in Proceedings of the Symposium "Analytical and Numerical Methods in Quaternions and Clifford Analysis", Seiffen, W.Sprössig and K. Gürlebeck eds., Technical University of Freiberg, 1997.

[McM99] A. McIntosh and M. Mitrea, Clifford algebras and Maxwell's equations in Lipschitz domains, Math. Meth. Appl. Sci., 22 (1999), 1599-1620. MR.1727215(2001c:30046)

[MM98] D. Mitrea and M. Mitrea, Uniqueness for inverse conductivity and transmission problems in the class of Lipschitz domains, Comm. Partial Differential Equations, 23 (1998), 1419-1448. MR1642603 (99f:35225)

[MMP97] D. Mitrea, M. Mitrea and J.Pipher, Vector potential theory on non-smooth domains in $\mathbb{R}^{3}$ and applications to electromagnetic scattering, J. Fourier Anal. and Appl., 3 (1997), no. 2, 131-192. MR.1438894 (99e:31009)

[MMS06] D. Mitrea, M. Mitrea and Q. Shi, Variable coefficient transmission problems and singular integral operators on non-smooth manifolds, J. Integral Equations Appl., 18 (2006), no. 3, 361-397. MR2269727 (2007k:35095)

[MMT01] D. Mitrea, M. Mitrea and M. Taylor, Layer Potentials, the Hodge Laplacian, and Global Boundary Problems in Nonsmooth Riemannian Manifolds, Mem. Amer. Math. Soc., Vol. 150, No. 713, 2001. MR.1809655 (2002g:58026)

[Mi02] M. Mitrea, Boundary value problems for Dirac operators and Maxwell's equations in nonsmooth domains, Mathematical Methods in the Applied Sciences, 25 (2002), no. 16-18, 1355-1369. MR.1949501 (2003k:35198)

[Mi01] M. Mitrea, Generalized Dirac operators on non-smooth manifolds and Maxwell's equations, Journal of Fourier Analysis and Applications, 7 (2001), no. 3, 207-256. MR1835281 (2002k:58046)

[Mi95] M. Mitrea, The method of layer potentials in electro-magnetic scattering theory on non-smooth domains, Duke Math. J., 77 (1995), no. 1, 111-133. MR.1317629 (96b:78035)

[Mi94] M. Mitrea, Clifford Wavelets, Singular Integrals, and Hardy Spaces, Lecture Notes in Mathematics, No.1575, Springer-Verlag, Berlin, Heidelberg, New York, 1994. MR.1295843 (96e:31005)

[MW10] M. Mitrea and M. Wright, Boundary Value Problems for the Stokes System in Arbitrary Lipschitz Domains, to appear in Astérisque, Societé Mathématique de France, 2011.

[Mu51] C. Müller, Über die Beugung elektromagnetischer Schwingungen an endlichen homogenen Körpern, Math. Ann., 123 (1951), 345-378. MR.0045030 (13:514c)

[Mu69] C. Müller, Foundations of the Mathematical Theory of Electromagnetic Waves, Springer-Verlag, Berlin, Heidelberg, New York, 1969. MR0253638 (40:6852)

[NS99] S. Nicaise and A.-M. Sändig, Transmission problems for the Laplace and elasticity operators: Regularity and boundary integral formulation, Math. Models Methods Appl. Sci., 9 (1999), 855-898. MR1702865 (2000i:35022)

[Re89a] S. Rempel, Corner singularity for transmission problems in three dimensions, Integral Equations Operator Theory, 12 (1989), 835-854. MR1018215 (91f:35082)

[Re89b] S. Rempel, Elliptic pseudodifferential operators on manifolds with corners and edges, pp. 202-211, in "Function Spaces, Differential Operators and Nonlinear Analysis," Pitman Res. Notes Math. Ser., Vol. 211, Longman Sci. Tech., Harlow, 1989. MR.1041119 (91b:58253)

[Rei93] M. Reissel, On a transmission boundary value problem for the time-harmonic Maxwell equations without displacement currents, SIAM J. Math. Anal., 24 (1993), 1440-1457. MR.1241153(94i:35186)

[Sa52] W.K. Saunders, On solutions of Maxwell equations in an exterior region, Proc. Nat. Acad. Sci. U.S.A., 38 (1952), 342-348. MR0053763 (14:823a)

[Seo97] J.K.Seo, Regularity for solutions of transmission problems across internal nonsmooth boundary, pp. 189-199 in Proceedings of Miniconference of Partial Differential 
Equations and Applications, (Seoul, 1995), Lecture Notes Ser., 38, Seoul Nat. Univ., Seoul, 1997. MR1449919 (98d:35043)

[St70] E.M. Stein, Singular Integrals and Differentiability Properties of Functions, Princeton Mathematical Series, No.30, Princeton University Press, Princeton, N.J., 1970. MR.0290095 (44:7280)

[Sh91] Z. Shen, Boundary value problems for parabolic Lamé systems and a nonstationary linearized system of Navier-Stokes equations in Lipschitz cylinders, Amer. J. Math., 113 (1991), 293-373. MR1099449 (92a:35133)

[Ve84] G. Verchota, Layer potentials and boundary value problems for Laplace's equation in Lipschitz domains, J. Funct. Anal., 59 (1984), 572-611. MR769382 (86e:35038)

[We52a] H.Weyl, Kapazität von Strahlungsfeldern, Math. Zeit., 55 (1952), 187-198. MR0049782 (14:225c)

[We52b] H. Weyl, Die natürlichen Randwertaufgaben im Aussenraum für Strahlungsfeldern beliebiger Dimensionen und beliebiger Ranges, Math. Zeit., 56 (1952), 105-119. MR0054524 (14:933g)

[Wi87] P. Wilde, Transmission problems for the vector Helmholtz equation, Proc. Roy. Soc. Edinburgh Sect. A, 105 (1987), 61-76. MR890043 (88g:35064)

Instituto de Matemáticas Unidad Cuernavaca, Universidad Nacional Autónoma de México, A.P. 273-3 Admon. 3, Cuernavaca, Morelos, 62251, México

E-mail address: emilio@matcuer.unam.mx

Department of Mathematics, Temple University, 1805 N. Broad Street, Philadelphia, Pennsylvania 19122

E-mail address: imitrea@temple.edu

Department of Mathematics, University of Missouri, Columbia, Missouri 65211

E-mail address: mitream@missouri.edu

Department of Mathematics, Computer Science and Economics, Emporia State UniVERSITY, EMPORIA, KANSAS 66801

E-mail address: qshi@emporia.edu 